\title{
Time-of-day optimality effects on eyewitness memory performance
}

Citation for published version (APA):

Yaremenko, S. (2020). Time-of-day optimality effects on eyewitness memory performance. [Doctoral

Thesis, Maastricht University]. Maastricht University. https://doi.org/10.26481/dis.20200918sy

Document status and date:

Published: 01/01/2020

DOI:

10.26481/dis.20200918sy

Document Version:

Publisher's PDF, also known as Version of record

\section{Please check the document version of this publication:}

- A submitted manuscript is the version of the article upon submission and before peer-review. There can be important differences between the submitted version and the official published version of record.

People interested in the research are advised to contact the author for the final version of the publication, or visit the DOI to the publisher's website.

- The final author version and the galley proof are versions of the publication after peer review.

- The final published version features the final layout of the paper including the volume, issue and page numbers.

Link to publication

\footnotetext{
General rights rights.

- You may freely distribute the URL identifying the publication in the public portal. please follow below link for the End User Agreement:

www.umlib.nl/taverne-license

Take down policy

If you believe that this document breaches copyright please contact us at:

repository@maastrichtuniversity.nl

providing details and we will investigate your claim.
}

Copyright and moral rights for the publications made accessible in the public portal are retained by the authors and/or other copyright owners and it is a condition of accessing publications that users recognise and abide by the legal requirements associated with these

- Users may download and print one copy of any publication from the public portal for the purpose of private study or research.

- You may not further distribute the material or use it for any profit-making activity or commercial gain

If the publication is distributed under the terms of Article $25 \mathrm{fa}$ of the Dutch Copyright Act, indicated by the "Taverne" license above, 


\section{Time-Of-Day Optimality Effects on Eyewitness Memory Performance}

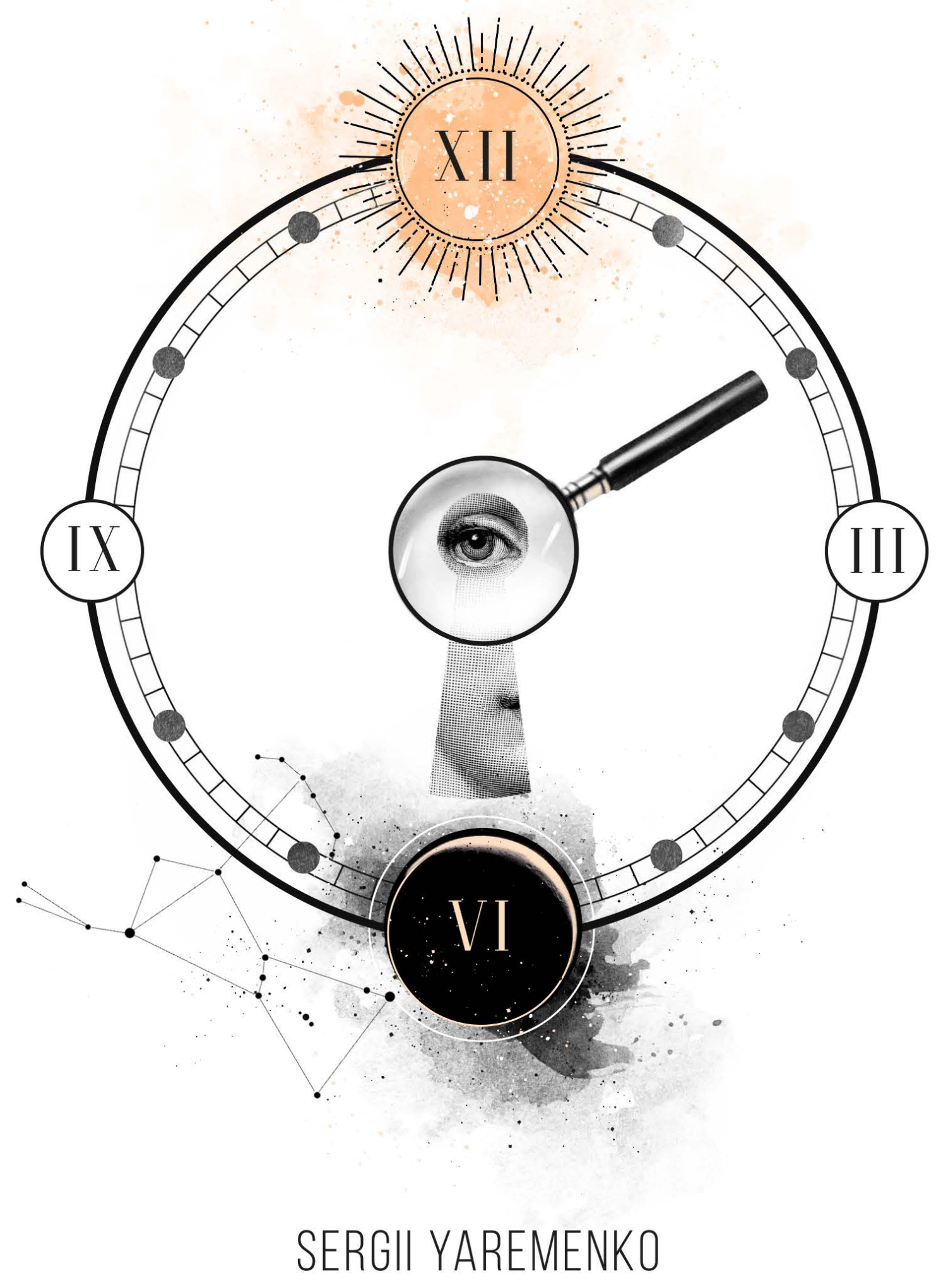


Time-of-Day Optimality Effects on Eyewitness Memory

Performance

Sergii Yaremenko 
(C) copyright Sergii Yaremenko, Maastricht 2020

\section{ISBN 9789464190281}

All rights reserved. No part of this publication may be reproduced, stored in a retrieval system or transmitted, in any form or by any means, electronic, mechanical, photocopying, recording or otherwise, without prior permission of the author or the copyright-owning journals for previous published chapters.

Cover designed by Rita Sus (amusedanimal@gmail.com; www.smallinkart.com) 


\title{
Maastricht University
}

\section{Time-of-Day Optimality Effects on Eyewitness Memory Performance}

\author{
Dissertation
}

To obtain the degree of Doctor of Philosophy from The University of Portsmouth and the degree of Doctor at Maastricht University, on the authority of Rector Magnificus Prof.dr. Rianne M. Letschert, according to the decision of the Board of Deans, to be defended in public on Friday 18th of September 2020 at 16.00 hours in Maastricht

by

Sergii Yaremenko 
Supervisors:

Dr. Melanie Sauerland

Maastricht University, the Netherlands

Prof. dr. Lorraine Hope University of Portsmouth, United Kingdom

Prof. dr. Henry Otgaar Maastricht University, the Netherlands

\section{Assessment Committee:}

Prof. dr. Marco Jelicic

Maastricht University, the Netherlands

Prof. dr. Graham Pike The Open University, United Kingdom

Dr. Paul Morris University of Portsmouth, United Kingdom

Prof. dr. Pekka Santtila New York University Shanghai, China 
This research is supported by a fellowship awarded from the Erasmus Mundus Joint Doctorate Program The House of Legal Psychology with Framework Partnership Agreement 2013-0036 and Specific Grant Agreement 2016-1339 to Sergii Yaremenko. 


\section{General Abstract}

Our body clock, also known as the circadian rhythm, regulates alertness and activity levels across the day and determines periods of optimal cognitive performance. The exact timing of peak performance may vary due to inter-individual differences in the functioning of our body clock: Morning types perform best in the morning, whereas evening times reach their peak performance in the evening. This pattern is known as the synchrony effect. There are numerous benefits in scheduling cognitively demanding activities in congruence with individual performance patterns. Recall and recognition performance have been shown to be better at the optimal compared to the non-optimal hours of the day. However, the role of the synchrony effect in eyewitness memory performance received little attention in the literature.

In the research programme presented in the current thesis, I aimed to investigate the effects of timeof-day optimality on memory performance in eyewitnesses. Across three single- and multi-session experimental studies, I (i) investigated the effect of time-of day optimality on accuracy and informativeness of free narratives and answers to cued questions about the mock crime (Chapter 1); (ii) explored time-of-day optimality effects on lineup identification performance and its postdictors (Chapters 1 and 2); (iii) tested time-of-day optimality effects in face recognition performance and memory for sources in which faces were encountered (Chapter 3), and (iii) explored possible synchrony effect patterns in the formation of false memories (Chapter 1 ).

In Chapter $2(N=103)$, I tested whether matching individual time-of-day preferences can be beneficial for accuracy and informativeness of eyewitness reports, accuracy of eyewitness identification decisions and postdictive value of confidence and decision times. I also investigated whether time-of-day optimality affects the formation of false memories. Time-of-day optimality did not affect performance in eyewitness reports and false memory rates. Identification accuracy in target-present lineups was unexpectedly higher at non-optimal compared to the optimal time of day. Highly confident choosers were significantly better calibrated in their confidence judgments at non-optimal compared to optimal hours. Decision times were predictive of accuracy only at the optimal time of day.

In Chapter $3(N=324)$, I further investigated the possibility of the synchrony effect in identification accuracy and its posdictors. Results showed no significant differences in identification accuracy between optimal and non-optimal sessions. In line with findings reported in Chapter 3, confidenceaccuracy relationship was stronger at the non-optimal time of day. Decision times were not predictive of accuracy.

In Chapter $4(N=91)$, I tested the possibility of the synchrony effect in face recognition performance and ability to discriminate between the contexts in which faces were encountered. Results showed no benefit in overall face recognition performance. Participants showed no benefit from optimal testing in terms of their ability to exclude familiar but irrelevant faces. These findings are novel in demonstrating 
that face recognition performance is not subject to synchrony effect patterns commonly reported in the literature.

Overall, the results of my experiments show no evidence for the synchrony effect in any of the investigated aspects of eyewitness memory performance. It can be concluded that in healthy young adult eyewitnesses, circadian troughs in cognitive performance are not sufficient to result in significant reduction of evidential value of testimony, providing that other encoding and retrieval conditions were optimal. My data suggest that eyewitnesses can take the presence of factors that impair cognitive performance into account and adjust their confidence judgments appropriately. 


\section{Table of Contents}

Chapter 1: Introduction

Introduction

The Circadian Rhythm and Chronotype

The Circadian Rhythm and Memory Performance

Circadian Variations in Eyewitness Memory Performance

Overview of the Current Thesis

Chapter 2: Circadian Variations in Recall and Identification Performance

Introduction

Method

Results

Discussion

Chapter 3: Disentangling Chronotype and Time-of-day Effects on Eyewitness

Identification Accuracy

Abstract

Introduction

Method

Results

Discussion

Method

Results

Discussion 
Summary of the Findings $\quad 68$

$\begin{array}{ll}\text { Interpreting Results } & 70\end{array}$

$\begin{array}{ll}\text { Task Difficulty } & 70\end{array}$

$\begin{array}{ll}\text { Encoding Instructions } & 72\end{array}$

$\begin{array}{ll}\text { Retrieval Instructions } & 73\end{array}$

$\begin{array}{ll}\text { Stimuli Type } & 73\end{array}$

$\begin{array}{ll}\text { Theoretical Contribution } & 73\end{array}$

The Synchrony Effect and Compensatory Resource Allocation Mechanisms $\quad 73$

The Circadian Rhythms and Metamemory $\quad 74$

Face Recognition and Fluctuations in Arousal $\quad 75$

Limitations

$\begin{array}{ll}\text { Concluding Remarks } & 77\end{array}$

$\begin{array}{lr}\text { References } & 79\end{array}$

$\begin{array}{ll}\text { Summary } & 94\end{array}$

$\begin{array}{ll}\text { Valorisation } & 96\end{array}$

$\begin{array}{lr}\text { Acknowledgments } & 98\end{array}$

$\begin{array}{ll}\text { Curriculum Vitae } & 101\end{array}$

$\begin{array}{ll}\text { Publications } & 103\end{array}$

$\begin{array}{ll}\text { Appendices } & 105\end{array}$

Appendix A: Supplementary materials (Chapter 2) 108

Appendix B: Supplementary materials (Chapter 4) 109

Appendix C: Favorable ethical opinion for research line 110 

CHAPTER 1

General Introduction 
Chapter 1 
Our body clock, also known as the circadian rhythm, is a biological mechanism that allows our body to adapt to cyclic changes occurring in the environment throughout the entire day (Halberg et al., 2003). This unique biological oscillator has an immense impact on virtually all aspects of our functioning ranging from purely physiological functions to various aspects of behaviour and cognition. The circadian rhythm coordinates the timing of numerous systems in our organism with 24-hour changes in the environment and keeps them in synchrony with each other (Hastings, Reddy, \& Maywood, 2003).

Sleep and wake cycle may be among the most apparent manifestations of the work of our body clock. Some other fluctuations caused by the circadian rhythm are so inherent in our daily functioning that we hardly pay any attention to them. They range from changes in appetite, body temperature and metabolism to daily fluctuations in our mood, arousal and ability to be at our best in physical and mental tasks (Reppert \& Weaver, 2002). Research has demonstrated advantages to making the timing of various activities congruent with our internal rhythms. This congruency can be beneficial in numerous aspects of our functioning, from planning sport trainings at optimum hours (Peek et al., 2017) to scheduling cognitively demanding activities at times of the day that facilitate top performance, such as later school time starts for adolescents (Kelley, Lockley, Foster, \& Kelley, 2015).

The legal arena represents another domain where optimal cognitive performance may be critical. Eyewitness testimony oftentimes constitutes the sole evidence in criminal cases. Therefore, the memory performance in eyewitnesses can play a crucial role in the administration of the law, with eyewitness errors potentially leading to wrongful convictions and other miscarriages of justice (Clark, Benjamin, Wixted, Mickes, \& Gronlund, 2015). However, little is known about the way prior research on circadian variations in cognitive performance translates into the eyewitness memory domain. For example, do eyewitnesses encode the details of crimes differently at different points of the day? Could the evidential value of identification decision vary as a function of the time of day when the perpetrator's face was encoded? Can we assist eyewitnesses in providing accurate statements by conducting an investigative interview at the peak of their cognitive performance? Are some hours of the day more optimal for administering lineups than others? These questions have received no attention in the (research) literature to date. The current thesis attempts to address this gap by taking the first steps in investigating circadian effects in eyewitness memory performance.

\section{The Circadian Rhythm and Chronotype}

Our heart produces around 108,000 beats per day with a rate that varies at different points of the day. The functioning and structure of cells involved in our stomach, liver, and pancreas vary from hour to hour, allowing our digestive system to function properly across the day. Over 50 different hormones are produced and circulate in our body, ensuring the proper functioning of the immune system and responses to stress, and making us feel sleepy at certain hours and alert at others. It is essential that these processes are coordinated with the environment and each other.

A clock-like mechanism known as the circadian rhythm (from the Latin circa, meaning 'around', and diem, meaning 'day') leaves little room for chaos in this highly complex system (Glass, 2001). A group of neurons located in the suprachiasmatic nucleus of the hypothalamus act as a central pacemaker, coordinating the functioning of multiple physiological, hormonal, and behavioural systems (Refinetti, 2006; Reppert \& Weaver, 2002). The circadian rhythm ensures that the timing of physiological and behavioural events is consonant with the demands the environment poses at each specific moment of the day. The circadian rhythm is entrainable, that is, it can be 'set' by the external cues such as light and food intake cycles and social interactions. However, in the absence of external time cues, for instance, in constant darkness, the circadian clock continues to maintain near-24-hour cycles, proving to be an endogenous, self- 
sustained biological clock that works with impressive precision (Borbély \& Achermann, 1999; Gaggioni, Maquet, Schmidt, Dijk, \& Vandewalle, 2014).

The exact timing of sleep, activity, and optimal performance periods can vary among individuals. These interindividual differences in sleep and activity timings are known in the literature as morningness-eveningness preference (Horne \& Östberg, 1976) or chronotype (Roenneberg, Wirz-Justice, \& Merrow, 2003). The chronotype dimension is best described as a continuum (Natale \& Cicogna, 2002). Evening types, who wake up and go to sleep late and prefer evening hours for their activity, are at one end of the continuum. Morning types, on the other hand, wake up and go to sleep earlier, and prefer to be active in morning hours (Horne \& Östberg, 1976; Roenneberg et al., 2007). Inter-individual differences in the circadian rhythm are not limited to sleep habits: Chronotype affects a plethora of factors of our functioning, such as the timing of peak hormone levels and body temperature, individual preferences in the timing of meals, and even differences in our thinking style (for a review, see Adan et al., 2012).

Chronotype is commonly studied using self-report tools that measure 24-hour changes in subjective alertness and preferences in the timing of sleep, physical, and cognitive activities. The Morningness-Eveningness Questionnaire (MEQ) constructed by Horne and Ostberg (1976) was the first such self-report tool and is still widely used in chronobiological research to measure of time-of-day preferences due to its sound psychometric properties. The MEQ consists of 19 questions about participants' sleeping habits, alertness levels at different points of the day, and preferred times for engaging in physically and cognitively demanding tasks (e.g., "At what time in the evening do you feel tired and in need of sleep?"). The MEQ score has been shown to correlate with daily changes in melatonin and cortisol levels, body temperature (Baehr, Revelle, \& Eastman, 2000; Horne \& Ostberg, 1976), sleep habits, and activity levels (Andrade, Benedito-Silva, \& Menna-Barreto, 1992; Bailey \& Heitkemper, 2001; Duffy, Rimmer, \& Czeisler, 2001).

A reduced version of the questionnaire, the rMEQ (Adan \& Almirall, 1991) includes five items from the full MEQ. The $r M E Q$ offers a convenient alternative to the longer version of the questionnaire without significant loss inter-item correlation and validity (Natale, Esposito, Martoni, \& Fabbri, 2006). The MEQ and its reduced version are the most commonly used tools to measure individual differences in the circadian rhythm (Adan et al., 2012). Some of the other self-report tools used to measure chronotype are the Munich Chronotype Questionnaire (Roenneberg et al., 2003), Diurnal Type Scale (Torsvall \& Åkerstedt, 1980), and Composite Scale of Morningness (Smith, Reilly, \& Midkiff, 1989). For a review of the psychometric properties in various questionnaires, see Di Milia, Adan, Natale, and Randler (2013).

\section{The Circadian Rhythm and Memory Performance}

The circadian rhythm can affect long-term memory performance via two distinct mechanisms. First, the circadian rhythm contributes to regulation of the timing of sleep (Van Dongen \& Dinges, 2003), which is a pre-requisite to any effortful cognitive activity (Antonenko, Diekelmann, Olsen, Born, \& Mölle, 2013; Cousins, Sasmita, \& Chee, 2017). Next, sleep plays a critical role in consolidation of newly encoded memories. Consolidation is essential making memory traces stable and immune to interference (for a review, see Rasch \& Born, 2013). A wealth of studies investigated the effects of sleep on different aspects of memory functioning that are of importance to the legal arena (e.g. Blagrove \& Akehurst, 2000; Morgan, Tamminen, Seale-Carlisle, \& Mickes, 2019; Payne et al., 2009; Stepan, Dehnke, \& Fenn, 2017). These sleeprelated effects in eyewitness memory performance are outside the scope of the current thesis.

Another way the circadian rhythm can affect memory relates to its role in maintaining cycles in alertness and arousal levels. Generally, our cognitive performance tends to be better during periods of high alertness. Thus, by regulating the levels of arousal in our waking life, the circadian rhythm determines hours that are optimal in terms of 4 
memory performance (Reppert \& Weaver, 2002). The effect of time-of-day optimality on eyewitness memory performance has received little attention in the research literature and is the primary focus of the current research programme.

Not everyone reaches their peak at the same hours of the day, and cognitive performance varies as a function of alignment of the task timing with individual's circadian arousal peaks, which are determined by chronotype. This pattern is referred to as the synchrony effect: Individuals with morning preference are at their best in the morning hours, whereas evening types peak in the evening (May, 1999; May \& Hasher, 1998). The synchrony effect patterns have been demonstrated across a large variety of cognitive domains, including attentional capacities and vigilance, inhibition of irrelevant responses, and performance on tasks that rely on short- and long-term memory (for a review, see Schmidt, Collette, Cajochen, \& Peigneux, 2007).

Episodic memory in particular appears to show synchrony effect patterns across a wide range of memory tasks, such as free recall test of memory performance. For example, in one experiment, morning- and evening-type participants encoded prose passages and provided reports of the studied material in their own words at either 9 AM, 2 PM, or 8 PM. Morning-type (but not evening-type) participants produced significantly more idea units from the passages at 9 AM compared to the later sessions, with small size of the observed effect (Petros, Beckwith, \& Anderson, 1990).

Another experiment compared performance on a stem completion task in the morning (8:00 AM and 9:00 AM) or in the early evening (between 5:00 PM and 6 PM) in younger morning-types and older evening-types (May, Hasher, \& Foong, 2005). Participants encoded the target words whilst performing a pleasantness-rating task. Subsequently, their memory for these words was tested in a stem completion task. Performance was better at the optimal time of day in both chronotype groups: Morning-type participants recalled more studied words in the morning, whereas evening types completed more stems correctly in the evening. These results show that cued recall performance also appears to be better at circadian peaks compared to circadian troughs.

Finally, recognition performance can also be susceptible to circadian variations in arousal. In an experiment on time of day effects on verbatim sentence recognition, morning-and evening-type participants encoded short stories either at 8 AM to 9 AM or at 4 PM to 5 PM (May, Hasher, \& Stoltzfus, 1993). After encoding short stories, participants were presented with old and new sentences and had to indicate whether each of them had been present verbatim in one of the original stories. In evening-type participants, hit rates were higher and false alarms were lower at their optimal compared to the non-optimal time of day. In morning-type older adults, the hit rates at the optimal and the non-optimal time of day were similar, whereas false alarms increased in the non-optimal session. The corrected recognition scores, computed as hits minus false alarms, confirmed overall better performance at the optimal time of day in both chronotype groups, with a small to medium size of the observed effect.

Non-optimal testing can also boost unwanted cognitive biases, such as false memories in the Deese/RoedigerMcDermott (DRM) paradigm (Deese, 1959; Roediger \& McDermott, 1995). In this paradigm, participants encode associatively related items (e.g., nurse, hospital, ill, medicine, stethoscope). When their memory is tested, participants oftentimes report studying the associatively related critical lure (e.g., doctor), even though the lure has not been presented to participants (Cann, McRae, \& Katz, 2011; Roediger \& McDermott, 1995; Roediger, Watson, McDermott, \& Gallo, 2001). The production of these memory illusions can follow the synchrony effect pattern in older adults, who show higher false memory rates at non-optimal compared to the optimal time of day (Intons-Peterson, Rocchi, West, McLellan, \& Hackney, 1999). These experiments are a part of a large body of research showing that the synchrony effect in memory 


\section{Chapter 1}

performance appears to be a robust phenomenon observed in various basic memory tasks (for other examples, see Puttaert, Adam, \& Peigneux, 2018; Ryan, Hatfield,, \& Hofstetter, 2002; Yang, Hasher, \& Wilson, 2007; Yoon, 1997). Circadian arousal is an important factor affecting the way we remember autobiographical events.

From a theoretical perspective, the synchrony effect is often construed as a cyclic variation in the amount of available cognitive resources (e.g., Nowack \& Van Der Meer, 2018). We subjectively experience circadian arousal cycles as changes in energy levels throughout the day (Cariou, Galy, \& Mélan, 2008; Thayer, 1987). The energy dimension of arousal is thought to reflect the amount of attentional resources available for any effortful mental activities we wish to engage in (Hirst \& Kalmar, 1987; Necka, 1997).

Attention plays a crucial role in the successful encoding of information, serving as a link between perception and memory (e.g., Chun \& Turk-Browne, 2007). The amount of attention paid to an autobiographical experience determines the extent to which the event can be successfully recalled later (Craik, Govoni, Naveh-Benjamin, \& Anderson, 1996) ${ }^{1}$. Encoding information in long-term memory is not merely a byproduct of paying attention to the stimulus. Rather, it largely depends on the degree of elaboration we engage in when processing the incoming stimuli (Craik \& Lockhart; 1972; Lockhart, 1992; Otten, Henson, \& Rugg, 2001). Shallow processing leads to weak encoding, whereas deep, or more elaborate processing results in better memory for the studied material (Craik, 1999; Craik \& Lockhart, 1972; Craik \& Tulving, 1975). Elaborate processing occurs when we make connections between individual elements of the incoming information (Bousfield \& Cohen, 1953; Tulving, 1962) or link the new information with pre-existing knowledge and experience (Craik; 2002; Hastie \& Kumar, 1979). Optimal attentional capacities at circadian arousal peaks allow us to engage in elaborate processing of incoming stimuli, which presumably results in stronger encoding of an event (Valdez, 2019).

In a similar vein, retrieval from long-term memory is an effortful activity and can be sensitive to factors that negatively affect our cognitive resources (e.g., Fernandes \& Moscovitch, 2000). To complete a difficult memory test, participants need to be able to pay proper attention to instructions and process them. Providing a detailed recollection of an incident can be a lengthy and effortful task that consumes cognitive resources. Even mnemonic techniques that aid recollection require some amount of cognitive capacities. For instance, effort is necessary in order to mentally reinstate the context of a to-be-remembered event. Accurate retrieval may also require suppressing unwanted responses, an ability that largely relies on the available cognitive resources (e.g., Jacoby, Woloshyn, \& Kelley, 1989; Kane \& Engle, 2000).

Our understanding of mechanisms that underlie circadian variations in memory performance may not be complete. Recent neuroscientific studies demonstrated that cortical and hippocampal areas involved in memory functioning can manifest their own circadian oscillations that function as an autonomous peripheral clock complementing the central pacemaker located in superchiasmatic nucleus (Eckel-Mahan \& Storm, 2009). It is likely that these intrinsic circadian rhythms in the hippocampus play an important role in the time-of-day fluctuations in memory functioning. However, the underlying cellular and molecular mechanisms of these peripheral oscillations and their actual effect on memory performance are yet to be explored (Snider, Sullivan, \& Obrietan, 2018).

The theoretical understanding of the mechanisms underpinning the synchrony effect is an interesting avenue for future research. While the primary focus of the current thesis lies in the applied field of eyewitness memory

\footnotetext{
${ }^{1}$ This statement relates primarily to conscious, explicit recollection; the interrelation between and implicit memory is more complex (e.g., Mulligan, 1998). 
performance, exploring manifestations of circadian fluctuations in arousal across various contexts can contribute to overall understanding of the underlying mechanisms behind the synchrony effect.

\section{Circadian Variations in Eyewitness Memory Performance}

Eyewitness testimony can be a double-edged sword in the criminal justice system. Accurate and informative testimony may lead to a breakthrough in investigation (Graham, 2003), whereas memory errors can result in wrongful convictions (Clark et al., 2015). Therefore, the study of human memory and factors that affect it are of high importance to the legal arena.

Decades of scientific research into the functioning of human memory provided insight into how we remember autobiographical events. Traditionally, experimental protocols in memory research relied on variations of the so-called verbal-learning paradigm. In this model, participants study lists of words (or, alternatively, prose fragments, drawings, photographs of objects etc.). Memorizing the word list itself, as well as each specific item on the list, serves as an experimental model of encoding an autobiographical experience (Kihlstrom, 2009). Participants retain the studied material for various intervals. After that, experimenters test participant's memory for the encoded experience. A free recall test is one example of generic memory tasks frequently used to test memory performance. In this test, participants are simply asked to report the experience they remember (e.g., "Write down the words that you studied earlier"). In another type of test known as cued recall, participants are provided with extra cues that aid retrieval (e.g., "Use the words that you studied to complete these word stems"). On a recognition test, a copy of a studied item serves as a cue (e.g., "Do you remember seeing this drawing?"). By manipulating conditions during encoding, retention or retrieval, researchers make inferences about the way various factors affect our memory.

The verbal-learning paradigm has proven to be an effective experimental model simulating human memory processes in laboratories of cognitive psychologists. Decades of research that relied on variations of the verbal-learning paradigm immensely increased our understanding of fundamental principles of memory functioning (Kihlstrom, 1996). The synchrony effect literature is also based on analogous methods of studying memory: By comparing memory for words, sentences, prose passages and objects at the optimal and non-optimal hours of the day, we now know that memory is best during peaks of circadian arousal (Intons-Peterson et al., 1999; May et al., 1993, 2005; Petros et al.,1990; Puttaert et al., 2018; Ryan et al., 2002; Yang et al., 2007; Yoon, 1997).

Is it reasonable to assume that memory for crimes could be subject to similar circadian variations in performance? As a matter of fact, there are numerous examples of classic discoveries about memory functioning that map well onto the eyewitness memory field. Such factors as retention interval, exposure duration or the age of the witness are now widely acknowledged as important contributors to memory performance in eyewitnesses (see, e.g., Loftus, 1975; Wells \& Olson, 2003). However, not all laboratory findings can be applied to the eyewitness memory domain in such a straightforward manner. Conditions that real eyewitnesses face can fundamentally differ from the protocol of a standard memory experiment. Witnessing a crime bears little resemblance to studying word lists or memorising prose passages. Furthermore, the nature of the memory queries in the context of criminal proceedings significantly differs from memory tests in a memory lab, and the costs of memory errors in investigative contexts are incomparable to mistakes in recognizing the wrong word in a standard memory experiment.

A set of methodologies known as the eyewitness memory paradigm was designed to address potential issues of ecological validity when applying findings of traditional episodic memory research to the eyewitnesses (Loftus, Miller, \& Burns, 1978). Instead of memorizing word lists or text fragments, participants in eyewitness memory studies may become 
mock witnesses to a (digitally recorded) staged crime. As a free recall test, they may be asked to provide free narratives of what they remember. Questions about the incident and people involved are an analogue of standard cued recall task (e.g., Brewer, Vagadia, Hope, \& Gabbert, 2018). When asking participants to identify the perpetrator from a photographic lineup, experimenters essentially test their recognition memory. Eyewitness memory research has significantly advanced our understanding of how our general knowledge about memory functioning applies to the eyewitness memory domain. Factors that relate to memory performance in eyewitnesses are traditionally classified into two broad categories: estimator variables, or factors outside of control of the criminal justice and law enforcement systems (e.g., viewing conditions, age of the eyewitness, levels of intoxication), and system or contro/ variables, which can be under control of the criminal justice system (e.g., the interviewing protocol, the lineup composition, eyewitness confidence etc.; Wells, 1978; Wells et al., 2020; Wells, Memon, \& Penrod, 2006).

As the circadian rhythm consistently appears to affect memory performance, time-of-day optimality could be among the potential estimator variables: Memory for crime may be affected by the levels of circadian arousal in the eyewitness at the time of encoding. In a similar way, the circadian arousal could potentially act as a system variable: Perhaps it is beneficial to obtain eyewitness statements and administer lineup identifications at hours of the day when witnesses are at the peak of their cognitive performance. The methodology of previously conducted research allows us to draw only limited conclusions in these directions. The eyewitness memory paradigm, however, offers adequate and valid methods of investigating the role of the circadian rhythm in eyewitness memory contexts.

To the best of my knowledge, only one study used the eyewitness memory paradigm to explore the possibility of circadian variations in eyewitness memory performance. In this experiment, mock eyewitnesses with either morning or evening chronotype were tested either at 10 AM or at 8 PM (Diges, Rubio, \& Rodriguez, 1992). They encoded a stimulus event depicting a traffic accident and provided free narratives and answers to cued questions about the witnessed event. Results were contradictory, with an overall pattern of better performance in the morning compared to evening in both chronotypes. Unfortunately, the paper does not report participants' mean MEQ scores, which would allow us to assess whether this tendency is related to the differences in the strength of time-of-day preference in each of the chronotype subgroups (e.g., it is possible that morning types showed a stronger preference for morning compared to the preference for the late hours in the evening types). Combined with the small sample size (i.e., 10 participants per experimental condition), ambiguous reporting of the recall coding method, unclear exclusion criteria (e.g., caffeine consumption and sleep prior to testing), I cannot draw confident conclusions about these findings.

\section{Overview of the Current Thesis}

The current programme of research presents some of the first attempts to test the synchrony effect patterns in eyewitness memory performance. Across three single- and multi-session experiments, I investigated whether (1) eyewitnesses provide more complete and accurate free narratives and answers to cued questions about the crimerelated event at their optimal compared to the non-optimal time of day (Experiment 1); (2) eyewitnesses make more accurate lineup identification decisions at the optimal compared to the non-optimal time of day (Experiments 1 and 2); (3) face recognition performance is higher at circadian peaks compared to circadian troughs (Experiment 3); and (4) eyewitnesses are better at discriminating faces they encountered at the crime scene from faces that were encoded in unrelated contexts (Experiment 3). I also examined whether false memory rates are higher at the non-optimal compared to the optimal time of day (Experiment 1). To the best of my knowledge, these are the first attempts to investigate these effects in the context of eyewitness memory performance. 


\section{Chapter 2: Circadian Variations in Recall and Identification Performance}

Previous research shows circadian-dependent time-of-day variations in recall and recognition performance. In Experiment 1, I tested whether matching individual time-of-day preferences can be beneficial for accuracy and informativeness of eyewitness reports, accuracy of eyewitness identification decisions, and postdictive value of confidence and decision times. I also investigated whether time-of-day optimality affects the formation of false memories. The hypothesis was that eyewitness reports provided at circadian peaks would be more detailed and more accurate compared to reports provided at circadian troughs. I also expected participants to make more accurate identification decisions in target-present and target-absent lineups during their optimal compared with the non-optimal time of day. In terms of spontaneous false memory production, I expected to replicate previous findings showing no time-of-day variations in false memory rates in younger adults.

\section{Chapter 3: Disentangling Chronotype and Time-of-day Effects on Eyewitness Identification Accuracy}

Experiment 2 aimed to further investigate possible time-of-day effects in eyewitness identification performance and its postdictors. A between-subjects design was used as opposed to within-subjects design in Experiment 1 to exclude the possibility of expectancy effects. The use of Amazon MTurk platform to recruit participants allowed me to achieve a more demographically diverse sample compared to standard undergraduate student population. Finally, I attempted to introduce bias into the identification procedure by exposing participants to mug shots of an incidental target. I expected higher identification accuracy in the optimal compared to the non-optimal sessions. I also hypothesised that non-optimal testing would magnify the potential biasing effects of mug shot exposure.

\section{Chapter 4: Circadian Effects on Face Recognition and Source Memory Performance}

In Chapter 4, I tested the possibility of synchrony effects in face recognition performance and ability to discriminate between the contexts in which faces were encountered. Participants encoded face stimuli embedded into two distinct contexts. One context linked faces to a crime-related scenario, whereas the other context presented faces in a neutral scenario. I presented participants with a standard face recognition test, expecting higher recognition performance at the optimal compared to the non-optimal time of day. Additionally, I administered a source recognition test, in which participants were asked to only select faces from the criminal scenario and exclude irrelevant faces from neutral context. The prediction was that non-optimal testing would impair participants' ability to exclude familiar but irrelevant faces encoded in the neural context.

\section{Chapter 5: General Discussion}

The thesis concludes with a general discussion (Chapter 5), in which I analyzed the findings of the current research programme in the view of the general theory of circadian variations in cognitive performance. I consider methodological differences between my experiments and previous synchrony effect studies that may underpin the discrepancy of my findings with the previously reported data. Importantly, these differences in experimental paradigms root in the specifics of conditions real-life eyewitnesses are exposed to. I discuss the issue of constraints of generality of standard laboratory research to applied settings, such as eyewitness memory contexts. 
Chapter 1 
CHAPTER 2

\section{Circadian Variations in Recall and Identification} Performance

Yaremenko, S., Sauerland, M., Hope, L. (2020) Time-of-day effects on eyewitness reports in morning and evening types. Manuscript submitted for publication.

Yaremenko, S., Sauerland, M., Hope, L. (2020) Circadian variations in eyewitness identifications: Identification performance is not affected by time-of-day optimality. Manuscript submitted for publication, revision invited. 


\begin{abstract}
Our performance varies throughout the day as a function of alignment with our circadian rhythms. In basic memory experiments, memory performance is best at circadian peaks as opposed to circadian troughs. The current experiment tested whether similar performance patterns can be observed in the context of eyewitness memory performance. One-hundred-and-three morning- and evening-type participants were tested twice, both at their optimal and the non-optimal time of day. After watching a stimulus film, participants provided a free report, answered cued questions, and identified individuals involved in the stimulus event in the morning and the evening hours. Additionally, I administered a visual version of the Deese/Roediger-McDermott (DRM) false memory paradigm. Contrary to my predictions, time of testing did not have a statistically significant effect on quantity and accuracy of eyewitness statements. Unexpectedly, identification accuracy in target-present but not target-absent lineups was better in non-optimal compared with optimal sessions. The decision time-accuracy relationship was undermined for choosers, when tested at the non-optimal time of day, whereas the confidence-accuracy relationship was not affected by testing optimality. The production of spontaneous false memories in the DRM paradigm was not affected by time-of-day optimality. My findings lend no support for the idea that performance of morning- and evening-type eyewitnesses can be higher at their optimal as opposed to the non-optimal time of day.
\end{abstract}


Life on earth revolves around a 24-hour daylight cycle, and most organisms on our planet, including humans, function in synchrony with this rhythm (Kyriacou \& Hastings, 2010). This means that the timing of various processes and events in our body is not random; rather, they are synchronized with the cyclic changes occurring in external environment. The underlying clock-like mechanism responsible for these changes is known as the circadian rhythm (from the Latin circa, meaning 'around', and diem, meaning 'day') and is regulated by a group of neurons located in the suprachiasmatic nucleus of the hypothalamus. It coordinates the functioning of multiple physiological, hormonal and behavioural systems, allowing the organism to adapt to daily changes in the environment by ensuring the proper timing of a large number of physiological and behavioural events (Gaggioni, Maquet, Schmidt, Dijk, \& Vandewalle, 2014). Some of the processes regulated by the circadian clock in humans include variations in body temperature, cardiac activity, hormone levels, and metabolism (Borbély \& Achermann, 1999).

The circadian clock also dictates the levels of physiological arousal at each specific moment of the day to maintain the optimum timing of rest and activity periods. To perform this important function, the circadian rhythm interacts with another process known as the homeostatic sleep pressure (Van Dongen \& Dinges, 2003). The sleep pressure phenomenon refers to the fact that arousal levels decrease and propensity to sleep increases as the day progresses. In lay terms, the more time we spend awake, the more tired and sleepy we feel. Circadian process and homeostatic process work together in regulating melatonin and cortisol levels to determine the propensity to physical and mental activities and sleep at each specific moment of the day (Borbély, Daan, Wirz-Justice, \& Deboer, 2016).

Not everyone's body clock runs to the same timings. On the contrary, people widely differ in their preferred time of the day for sleep and activity, as determined by their chronotype, or time-of-day preference (Horne \& Östberg, 1976; Levandovski, Sasso, \& Hidalgo, 2013). Morning types, often referred to as 'larks', prefer waking up at early hours and find it difficult to stay awake in the evening. Evening types, or 'owls', prefer to go to sleep in the late hours and have difficulties getting up early in the morning. Intermediate types, who comprise around $60 \%$ of the population, show no strong morning or evening preference (Adan et al., 2012).

Cognitive performance largely depends on whether or not the actual time of day is aligned with individual time-of-day preference (Schmidt, Collette, Cajochen, \& Peigneux, 2007). Morning types reach their functional peak in the morning, as opposed to evening types, who are at their best in the evening hours. This phenomenon is known as the synchrony effect and it has been shown to affect inhibition of distractors, non-relevant thoughts and unwanted responses (May, 1999; May \& Hasher, 1998), automatic application of stereotypes and other judgmental heuristics (Bodenhausen, 1990), implicit memory (May, Hasher, \& Foong, 2005), and accessibility of information from semantic memory (Anderson, Petros, Beckwith, Mitchell, \& Fritz, 1991).

This synchrony effect is also observed in long-term memory performance. One study investigated whether matching or mismatching time of testing to participants' time-of-day preference affected immediate recall of prose passages (Petros, Beckwith, \& Anderson, 1990). Morning types recalled more idea units at 9 AM (optimal) compared to the afternoon and evening sessions (non- 
optimal), whereas no optimality effect was observed in evening-type participants. In another experiment, participants encoded a series of paragraph-length stories and then performed a verbatim sentence recognition task. The performance of evening participants improved from morning to afternoon, whereas performance of morning types was better in the morning, compared to the afternoon (May, Hasher, \& Stoltzfus, 1993).

Overall, the body of research on circadian variations in cognitive performance suggests that time-of-day optimality is an important factor that can affect cognitively demanding activities. The legal arena represents a domain where optimal cognitive performance may be critical. Eyewitness testimony and identification procedures often feature as important evidence in criminal cases. Therefore, the performance of eyewitnesses can play a crucial role in the administration of the law, with memory errors potentially leading to wrongful convictions and other forms of miscarriages of justice (Clark, Benjamin, Wixted, Mickes, \& Gronlund, 2015). Yet the possible effects of time-of-day optimality on eyewitness memory performance have received no attention in the research literature to date.

Prior evidence in support of time-of day effects in long-term memory suggests that eyewitness memory performance may be subject to typical synchrony effect patterns. However, the nature of the stimuli and tasks employed in the previous studies showing the synchrony effect in memory performance is quite different from tasks and stimuli real-life eyewitnesses encounter. Specifically, participants in previous studies showing synchrony effects in memory performance encoded word lists, sentences, short stories and pictures, as opposed to details of a crime-related event and people involved in it. An eyewitness memory paradigm that addresses these issues of ecological validity is necessary to clarify how the prior findings translate into eyewitness memory field.

To the best of my knowledge, only one published study used an eyewitness memory paradigm to test the possibility of circadian variations in eyewitness memory performance (Diges, Rubio, \& Rodriguez, 1992). Mock eyewitnesses with morning or evening chronotype came to the lab at 10 AM or at 8 PM. They encoded a stimulus film depicting a traffic accident and provided free narratives and answers to cued questions about the witnessed event. Contrary to predictions of the synchrony effect literature, results showed an overall pattern of better performance in the morning compared to evening regardless of chronotype. However, the paper did not report participants' mean scores on the Morningness-Eveningness Questionnaire (MEQ). I therefore cannot assess whether this tendency was related to possible differences in strength of time-of-day preference in each of the chronotype groups. More specifically, morning types might have shown a stronger preference for the morning compared to evening types' preference for the late hours. Furthermore, the sample size was very small with 10 participants per experimental condition and the method of coding and exclusion criteria were unclear.

In light of such a limited literature, the current experiment sought to further investigate the role of time of testing on eyewitness memory performance. I used a mock eyewitness identification paradigm, in which participants watched stimulus films depicting a staged crime and were subsequently tested using recall and recognition tasks relevant to the eyewitness context. First, I asked participants to provide free recall narratives about the event. Then, all participants completed a set of cued questions 
about the event and the people involved. Based on past research showing superior recall during circadian peaks (Petros et al., 1990), I hypothesized that participants would provide more complete and accurate responses when tested at the optimal compared to the non-optimal time of day. Finally, participants were asked to identify the individuals they saw in the film from lineups. Based on prior evidence showing superior recognition performance during circadian peaks (Intons-Peterson, Rocchi, West, McLellan, \& Hackney, 1999; May et al., 1993), I expected participants to make more accurate identification decisions at the optimal compared to the non-optimal time of day.

Additionally, I investigated how chronotype synchrony affects postdictors of identification accuracy, namely post-decision confidence and decision times. Typically, accurate choosers make their decisions with more confidence than inaccurate choosers, whereas such associations between confidence and accuracy do not exist for nonchoosers (Brewer \& Wells, 2006; Dunning \& Stern, 1994; Sauerland \& Sporer, 2007; Sporer, Penrod, Read, \& Cutler, 1995). The postdictive value of confidence can be lowered in situations when encoding, retention and retrieval conditions are suboptimal (Deffenbacher, 1980; Sauer \& Hope, 2016). I tested the idea that chronotype asynchrony may be among such conditions. I predicted that testing participants at their preferred times would strengthen the confidence-accuracy relationship in choosers. More specifically, I expected confident choosers to be more accurate than non-confident choosers and this relationship to be stronger at the optimal compared to the non-optimal time of day. I did not expect to observe this relationship for non-choosers.

Similarly to post-decision confidence, research consistently shows a negative relationship between identification accuracy and response time in choosers but not non-choosers (Brewer \& Wells, 2006; Dunning \& Stern, 1994; Sauerland \& Sporer, 2007). I was interested in whether testing optimality can act as a moderator of this decision-time-accuracy relationship. Only one study investigated the effect of testing optimality on long-term memory access speed (Anderson et al., 1991). In this experiment, participants with morning or evening preference were asked to make judgements about word pairs. The testing sessions were scheduled at 9:00, 14:00 and 20:00. Results showed a typical synchrony effect pattern, that is, reaction times decreased across the day for evening types and increased for morning types. Based on these findings, I hypothesised that inaccurate choosers will take longer to make their decisions than accurate choosers, but even more so at the non-optimal compared to the optimal time of day.

The architecture of our memory processes might sometimes result in spontaneous development of false memories for details that were not present at the crime scene (Otgaar, Howe, Brackmann, \& Smeets, 2016). In laboratory, such automatic memory illusions can be studied with the Deese-Roediger-McDermott (DRM) paradigm (Roediger \& McDermott, 1995). In this paradigm, participants encode items (e.g., bedroom, pillow, rest, dream) that are associatively related critical lure(s) (e.g., sleep). Though the critical lures are not actually presented, a robust finding is that participants claim to remember these associatively related items Cann, McRae, \& Katz, 2011; Roediger, Watson, McDermott, \& Gallo, 2001). Time-of-day optimality has also been shown to affect the formation of spontaneous false memories. Interestingly, false memory rates appear to be higher during the optimal 
compared to the non-optimal hours of the day in older but not younger adults (Intons-Peterson et al., 1999). However, the absence of time-of-day variations in younger adults has not been replicated to date. Additionally, one limitation of this study is that age was fully confounded with chronotype, that is, all morning-type participants were older adults and all evening types were younger adults. I was interested in replicating the findings of Intons-Peterson et al. (1999) regarding false memory production in younger adults across the day and extending their research by testing whether a similar pattern of results would be obtained in a sample consisting of both morning- and evening-type young adults.

\section{Method}

\section{Participants}

I conducted an a priori power analysis for a repeated-measures within-factors ANOVA with G*Power v3.1 (Faul, Erdfelder, Buchner, \& Lang, 2009; Faul, Erdfelder, Lang, \& Buchner, 2007). Based on the data reported in previous studies on circadian effects in memory performance (May et al., 1993; Petros et al., 1990), I expected a small to medium effect size. Hence, I used an effect size $f$ of 0.18 , alpha error probability of .05 and a power of .95 , resulting in a required sample size of 103 . To achieve the planned sample size, I pre-screened 203 individuals who expressed interest in participation in the experiment for their circadian typology using the short form of Morningness-Eveningness Questionnaire (rMEQ; Adan \& Almirall, 1991). One-hundred-and-three pre-screened participants whose rMEQ score was 12 and lower (evening types) and 17 and above (morning types) were invited to participate (15 male, 87 female, 1 unspecified; age 18 to $58, M=22.6, M d n=22$ years). The sample consisted of university students $(n=98)$ and members of the general public $(n=4)$. About half of the sample consisted of evening-type $\left(54.3 \%, n=56, M_{\text {rMEQ }}=9.82, S D_{\text {rMEQ }}=1.88\right)$ and morning-type participants $(45.6 \%, n=47$, $\left.M_{\text {rMEQ }}=18.6, S D_{\text {rMEQ }}=1.50\right)$. Participants were native Dutch $(n=56)$, German $(n=26)$ or English $(n=21)$ speakers. I recruited only Caucasian participants to avoid cross-racial bias in the identification task (Meissner \& Brigham, 2001; Wilson, Berstein, \& Hugenberg, 2016).

\section{Materials}

Morningness-eveningness scales. I used the rMEQ (Adan \& Almirall, 1991) to classify participants into morning- and evening-type categories. The rMEQ consists of five items drawn from the original full 19-item Morningness-Eveningness Questionnaire (MEQ) developed Horne and Östberg (1976). Both MEQ and its reduced version are commonly used to assess individual differences in diurnal preferences (Adan et al., 2012; Di Milia, Adan, Natale, \& Randler, 2013). The use of the shorter scale allowed me to distract participants' attention from the main hypothesis by combining the rMEQ items with filler questions about eating habits (e.g., "When you get up in the middle of the night, how often do you snack?"). The rMEQ score ranges between four and 25, with high scores referring to stronger morningness preference. Considering the debate around the arbitrariness of the cutoffs suggested by the authors of questionnaires measuring diurnal preferences (Caci, Deschaux, Adan, \& Natale, 2009), I adopted cut-offs of $\leq 12$ for evening types and $\geq 17$ for morning types, as opposed to originally suggested $\leq 11$ for evening types and $\geq 18$ for morning types (Adan \& Almirall, 1991). In my decision to adopt more 
lenient cutoffs, I was guided by the view that chronotype is a continuous rather than a dichotomous construct.

Additionally, I administered the full MEQ post-hoc following all the experimental manipulations as an extra validation of our classification of participants into chronotype groups. To establish test-retest reliability, I extracted participants' responses to the five rMEQ items from a full version of the MEQ questionnaire that was administered at the end of the experiment. The results showed excellent testretest reliability, $r(101)=.92, p<.001$.

Stimulus films. Two different stimulus films depicting the theft of a wallet were used. The films differ in the details of the event, the environment, and the actors. Film 1 (adapted from Sauerland, Krix, van Kan, Glunz, \& Sak, 2014) depicts a theft taking place at a bar. Four amateur actors (2 male, 2 female, 22-58 years old) appear in Film 1. In stimulus Film 2 (adapted from Brackmann, Sauerland, \& Otgaar, 2019) the theft occurs in a university communal area. Three amateur actors (2 male, 1 female, 21-26 years old) appear in Film 2.

Photo lineups. Six-person target-absent and target-present simultaneous lineups were constructed for each of seven targets (Film 1; one thief, one victim, two bystanders; Film 2: one thief, one victim, one bystander). Each lineup included six shoulder-up photographs that were arranged in two rows of three pictures and labelled 1 to 6 . The target positions for the perpetrator, victim, bystander 1 , and bystander 2 lineups in Film 1 were 4, 5, 4, and 3, respectively. The target positions for the perpetrator, victim, and bystander in Film 2 were 3, 6, and 4, respectively. In target-absent lineups, the targets were replaced with photographs of another foil. The effective lineup sizes (Tredoux's Es) were established in a pilot study, in which 19 to 38 mock witnesses (total $N=219$ ) were presented with a description of the target and chose a lineup member that best matches the description (Tredoux, 1999). Tredoux's Es ranged from 3.6 to 5.6.

DRM Task. To induce spontaneous false memories, I used four black-and-white pictures depicting scenes of a classroom, a beach, a funeral and a wiretap operation adopted from Moritz, Woodward, and Rodriguez-Raecke (2006). The pictures were designed in such a way that each of the scenes contained 12 items that are typical for the context of the respective scene. For instance, a scene of a classroom depicted a teacher, students, a blackboard etc. Further, eight thematically related items were excluded from each scene (e.g., a pointer or a sponge were excluded from a scene of a classroom). These eight items served as critical lures in the recognition task. These materials have been successfully used to induce spontaneous false memories (e.g., Peters et al., 2012; Otgaar, Howe, Peters, Smeets, \& Moritz, 2014).

The scenes differed in emotional valence (neutral, positive, negative and delusional paranoid). However, I did not have reasons to predict that time-of-day optimality would affect the formation of spontaneous false memories differently depending on the emotional valence of the scene, nor was there a significant interaction with testing optimality when this factor was entered as a predictor into a statistical model. Therefore, this factor will not be discussed any further. 


\section{Design}

The experiment used a within-participants design, with time-of-day optimality serving as predictor. Each participant was tested both at their optimal and the non-optimal time of day. The order of optimality conditions (optimal - non-optimal versus non-optimal - optimal) was counterbalanced to control for potential learning effects.

In each of the sessions, participants encoded one of the two stimulus events. The order of presentation of stimulus films (Film 1 - Film 2 versus Film 2 - Film 1) was counterbalanced across optimality conditions. In terms of recall performance, the number of correct details provided by participants and accuracy rates served as dependent variables.

Participants also made seven identification decisions across two testing sessions. Overall, 54.8 $\%$ of lineups presented to participants were target-present and $45.2 \%$ were target-absent. The distribution of target-presence across the optimality conditions was such that $52.8 \%$ of lineups presented in the optimal testing condition and $56.7 \%$ of lineups in the non-optimal condition were target-present. Identification accuracy (accurate versus inaccurate), post-decision confidence (on an 11point scale ranging from 0-100\%, with intervals marked in 10\% steps), and decision times (measured in ms) served as outcome variables.

Finally, in each session, participants were presented with the DRM task designed to induce spontaneous false memories. In each session, participants were presented with two of the four DRM stimuli. The six possible combinations of pairs of stimuli were counterbalanced across the optimality conditions. The proportions of 'yes' responses to presented items (hit rates), false alarms to nonpresented critical lures and non-presented non-related controls served as the outcome variables.

\section{Procedure}

Pre-screening. Participants were recruited using advertisements on a university notice board and by actively handing out flyers. I told participants that the study concerned the effects of eating and caffeine-consumption habits on long-term memory in individuals with morning and evening time-of-day preference. Prospective participants who believed they had a morning or an evening preference contacted the research team, and were asked to fill out the pre-screening questionnaire to determine their eligibility to participate. The questionnaire consisted of five rMEQ items intermixed with filler questions concerning participants' eating habits included to provide additional support for the cover story. Participants with $\mathrm{rMEQ}$ scores $\leq 12$ (evening types) or $\geq 17$ (morning types) were invited to participate in the main experiment. They were instructed to exclude alcohol or caffeine-containing products and sleep a minimum of six hours prior to testing.

Main experiment. Participants were tested individually on two separate occasions, once in the morning (between 8:00 and 10:00) and once in the evening (between 19:00 and 21:00). The two testing sessions took place with an interval of a minimum of 36 hours to avoid possible fatigue following a nonoptimal session. The protocol for the two sessions was analogous, except where specifically indicated.

First, participants watched one of the two stimulus films. I instructed participants to pay close attention to every detail, as they would later be asked to act as an eyewitness. Immediately after the 
film, participants provided a free narrative of what they remembered about the event. Specifically, I asked participants to report all the details they remembered about the incident, including the sequence of actions and events. I also asked them to describe the appearance of the people involved. I instructed participants to make their report as complete and accurate as possible and discouraged them from guessing. Participants were given unlimited time to provide the free narratives.

After providing free narratives, participants went on to answer blocks of cued questions about the event and the people involved in the incident. First, they were presented with nine cued recall questions about the event (e.g., "Describe any interactions the thief/thieves has/have with the other people in the film"). Next, I presented participants with a schematic of the crime scene with people involved in the incident represented as silhouettes and informed them that they would be asked answer questions about each person they saw in the film. Participants were presented with three (Film 1) or four (Film 2) blocks of cued questions about the appearance of each of the persons involved in the incident, including their age, height, build, clothing etc. Blocks of questions about each of the persons involved in the event were presented separately in the following order: thief, victim, bystander 1, and (in case of Film 1) bystander 2. For each of the blocks, I cued participants with the schematic of the crime scene, where the silhouette of the respective person was highlighted (i.e., the block of questions about the bystander was preceded with a schematic of the crime scene with highlighted silhouette of the bystander).

All the recall instructions and questions were presented in participants' native language. For the complete list of cued questions, see Appendix A. Participants were given a choice to provide responses in their native language.

Free reports and answers to cued questions were followed by a 30-minute interval to avoid potential verbal overshadowing effect in identification tasks (Meissner \& Brigham, 2001). During this interval, participants filled in either the Pittsburgh Sleep Quality Index (Session 1; Buysse, Reynolds, Monk, Berman, \& Kupfer, 1989) or demographic questionnaires (Session 2), followed by a visual version of the DRM paradigm task (both sessions).

In the DRM task, I informed participants that they would see two pictures and asked them to attend closely to each of them as they would be asked questions about them later. Each of the pictures was then presented for 40 secs. The order of the pictures was randomized. After encoding the pictures, participants engaged in a filler task (tetris) for 5 minutes. At test, participants were presented with two lists of items, presented verbally, corresponding to each presented picture. Participants were asked to indicate whether they saw each of the items in the respective picture. Each of the lists contained 24 items, 12 of which were present in the original picture, eight were non-presented thematically related lures ${ }^{2}$, and four were non-presented unrelated controls. The items were presented in a random order.

\footnotetext{
${ }^{2}$ Retrospectively, I realized that for one of the pictures, participants were presented with seven instead of eight critical lures due to a program error. To control for this, the proportion of correct responses rather than their number was entered as the outcome variable in the analyses.
} 
Finally, participants were asked to identify each of the persons they saw in the film from a sixperson simultaneous lineup. The perpetrator lineup was always presented first. The remaining two or three lineups, respectively, appeared in a random order. Participants were informed that the targets may or may not be present in the lineups and were encouraged to select the "Not present" option if they were not sure or didn't know. Decision times for each identification decision were recorded automatically. After each decision, participants indicated their confidence.

At the end of the second session, participants filled out the full version of the MEQ as an extra control of the chronotype classification. Participants received either gift vouchers worth $€ 27.50$ or participation credit in return for participating and were debriefed via email upon completion of data collection.

\section{Recall coding}

Following Wright and Holliday (2007), I developed a scoring template for both stimulus events. Each information unit from the events was categorized as an Action (A), Person (P), or Object/Setting $(\mathrm{O} / \mathrm{S})$ detail. For example, a stimulus film sequence of a perpetrator taking a wallet from the table was coded as "The guy (1-P) took (1-A) the wallet (1-O/S) from the table (1-O/S)". The scoring templates contained 314 details for Film 1 and 298 details for Film 2.

Details reported in free narratives and answers to cued questions were coded against the template for accuracy. A detail was coded as correct if it was present in the stimulus event and described correctly. Details that were present in the stimulus event but described incorrectly were coded as incorrect. Details described by participants that were not present in the stimulus event were coded as fabricated. Subjective responses (e.g., "The girl looked sad") were excluded from analyses.

To establish inter-coder reliability, for each of the three languages, seven randomly selected statements from both stimulus films were coded by two independent scorers. Inter-rater reliability coefficients (kappas) ranged from $\mathrm{k}=.70$ to $\mathrm{K}=.87, \mathrm{ps}<.001$, indicating substantial to almost perfect strength of agreement (Sim \& Wright, 2005). When computing total accuracy rates, each detail provided by participants was counted once across free and cued recall.

\section{Results}

Data analyses. One participant showed an inconsistent classification according to rMEQ (the score of 12, i.e., an evening type) and the full version of the questionnaire, where he scored 57 (almost reaching the morningness cut-off of 59). Data for this participant were excluded from analyses. Two participants failed to return to laboratory for the second session. I excluded their data from the analyses of performance in recall and DRM tasks but included their lineup identification responses from the session they attended in the GEE analyses.

Effect of time-of-day optimality on quantity of details and accuracy rates in recall tasks. I conducted paired-samples t-tests to determine whether there was a statistically significant mean difference in quantity of details provided by participants in recall tasks. Additionally, I computed JZS Bayes factors to assess a degree to which the data favors the null compared with the alternative hypothesis (Jeffreys, 1961). Table 1 shows that participants provided a large number of details both in 
the free recall and the cued questioning phase. Accuracy was generally high, but significantly higher in free than cued recall, $t(99)=21.62, p<.001, d=2.16$. Contrary to our hypothesis, there was no statistically significant effect of time-of-day optimality on the number of correct details reported during free recall, $t(99)=1.13, p=.262, d=0.11, B F_{01}=2.82$, or in answers to cued questions, $t(99)=0.62, p=$ $.538, d=0.06, B F_{01}=5.15$. The Bayes factors suggest weak to moderate evidence in support of the null hypothesis. Testing optimality also did not have an effect on accuracy in free recall, $t(99)=-0.50, p=$ $.618, d=-0.05, B F_{01}=12.84$ or in answers to cued questions, $t(99)=0.46, p=.647, d=0.05, B F_{01}=6.05$. The Bayes factors indicate substantial to strong evidence in support of the null hypothesis. Indeed, accuracy in optimal and non-optimal sessions were strikingly similar.

Table 1

Mean number of correct and incorrect details reported by participants and overall quantity and accuracy rates in free recall and cued recall tasks as a function of time of testing

\begin{tabular}{|c|c|c|c|c|c|}
\hline & & \multicolumn{4}{|c|}{ Time of testing } \\
\hline & & \multicolumn{2}{|c|}{ Optimal } & \multicolumn{2}{|c|}{ Non-optimal } \\
\hline & & $M$ & $(S D)$ & M & (SD) \\
\hline \multirow[t]{4}{*}{ Free recall } & Correct & 72.04 & 18.83 & 70.28 & 19.04 \\
\hline & Incorrect & 3.08 & 2.88 & 2.79 & 2.48 \\
\hline & Total quantity & 75.98 & 19.91 & 73.75 & 19.86 \\
\hline & Accuracy rate & .95 & .04 & .95 & .04 \\
\hline \multirow[t]{4}{*}{ Cued recall } & Correct & 56.45 & 11.03 & 55.60 & 10.65 \\
\hline & Incorrect & 8.35 & 3.28 & 8.54 & 3.81 \\
\hline & Total quantity & 64.98 & 11.54 & 64.37 & 11.64 \\
\hline & Accuracy rate & .87 & .05 & .86 & .05 \\
\hline \multirow[t]{4}{*}{ Total recall } & Correct & 101.43 & 17.87 & 99.51 & 16.44 \\
\hline & Incorrect & 10.27 & 4.40 & 10.20 & 4.49 \\
\hline & Total quantity & 112.46 & 19.12 & 110.39 & 17.11 \\
\hline & Accuracy rate & .90 & .04 & .90 & .05 \\
\hline
\end{tabular}

Effect of time-of-day optimality on identification accuracy, choosing and other lineup outcomes. Lineup identification outcomes were analysed with the help of the generalized estimating equations (GEE) models. GEE models are able to handle correlated data structures and thus can be employed to analyse binary outcomes collected from repeated measurements of the same individual (Zeger, Liang, \& Albert, 1988). The GEE model allowed us to include all seven measurements from each participant in the same model. An alpha level of .05 was used for all inferential analyses. 
Table 2 displays frequencies and proportions of different lineup outcomes for each of the lineups. I conducted GEE analyses to establish the effect of time-of-day optimality (optimal versus nonoptimal) and target presence (target-present versus target-absent) on identification accuracy (accurate versus inaccurate). I entered optimality, target presence and their two-way interaction as predictors in the model and deleted non-significant terms stepwise. The final model included a significant Optimality $x$ Target-Presence interaction, Wald $\chi^{2}(1)=3.93, p=.048$. Simple slopes analyses revealed that participants made more accurate decisions at the non-optimal compared to the optimal time of day in target-present, Wald $\chi^{2}(1)=5.92, \operatorname{Exp}(B)=1.60, p=.015$, but not target-absent lineups, Wald $\chi^{2}(1)=$ $0.17, \operatorname{Exp}(B)=0.92, p=.680$. That means, in target-present lineups, participants were 1.6 times more likely to make an accurate decision at non-optimal compared to the optimal time of day (corresponding to a small effect size, Chen, Cohen, \& Chen, 2010). This finding was contrary to my expectations.

Following up on my unexpected findings, I tested whether lineup selection rates were affected by testing optimality. Optimality (optimal versus non-optimal), target-presence (target-present versus target-absent) and their two-way interaction were entered as predictors in the model; choosing (selection versus rejection) served as the outcome. The analysis revealed a significant Optimality x Target Presence interaction, Wald $\chi^{2}(1)=4.78, p=.029$. Simple slopes analyses showed that participants were less likely to make a positive identification decision at the optimal compared to the non-optimal time of day in target-present, Wald $\chi^{2}(1)=14.21, \operatorname{Exp}(B)=0.52, p<.001$, but not target-absent lineups, Wald $\chi^{2}(1)=0.06, \operatorname{Exp}(B)=1.05, p=.802$. The odds ratio of 0.52 corresponds to a small effect size (Chen et al., 2010).

Finally, I tested whether optimality affected choosing rates for targets as opposed to foils differentially. For this purpose, I conducted two GEE analyses with target and foil selections (selection versus no selection) as the outcome variables. There was a significant effect of optimality on target selection: Participants were more likely to select the target at the non-optimal compared to the optimal time of day, Wald $\chi^{2}(1)=5.75, \operatorname{Exp}(B)=1.58, p=.016$, whereas the effect of optimality on foil selections was not statistically significant, $p=.488$.

Table 2

Frequencies and proportions of different lineup outcomes as a function of time of testing

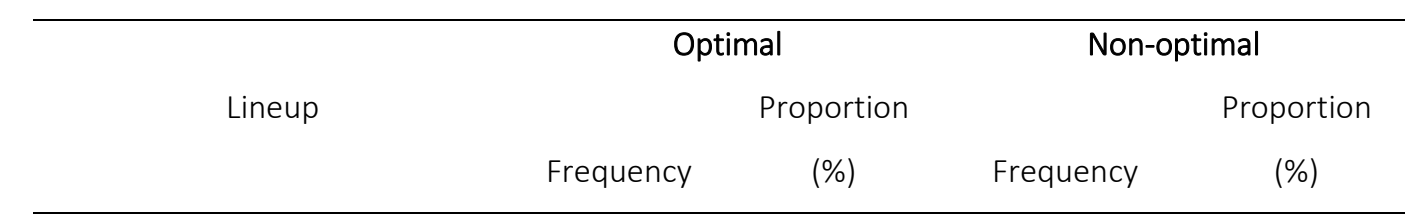

\section{Film 1 Thief}

Target-present

$\begin{array}{lcccc}\text { Targets choices } & 23 & 76.7 & 30 & 81.1 \\ \text { Foil choices } & 4 & 13.3 & 4 & 10.9 \\ \text { False rejections } & 3 & 10 & 3 & 8 \\ \text { otal } & 30 & 100 & 37 & 100\end{array}$


Target-absent

$\begin{array}{lcccc}\text { Correct rejections } & 10 & 55.6 & 8 & 53.4 \\ \text { Foil choices } & 8 & 44.4 & 7 & 46.6 \\ \text { otal } & 18 & 100 & 15 & 100\end{array}$

\section{Film 1 Victim}

Target-present

Target choices

10

29.4

21.2

Foil choices

8

23.6

42.4

False rejections

16

47

36.4

Total

34

100

100

Target-absent

$\begin{array}{lcccc}\text { Correct rejections } & 9 & 64.3 & 11 & 57.9 \\ \text { Foil choices } & 5 & 35.7 & 8 & 42.1 \\ \text { tal } & 14 & 100 & 19 & 100\end{array}$

\section{Film 1 Bystander A}

Target-present

$\begin{array}{lcccc}\text { Target choices } & 8 & 47.1 & 22 & 64.7 \\ \text { Foil choices } & 0 & 0 & 5 & 14.7 \\ \text { False rejections } & 9 & 52.9 & 7 & 20.6 \\ \text { al } & 17 & 100 & 34 & 100\end{array}$

Target-absent

$\begin{array}{lcccc}\text { Correct rejections } & 17 & 54.8 & 9 & 50 \\ \text { Foil choices } & 14 & 45.2 & 9 & 50 \\ \text { Total } & 31 & 100 & 18 & 100\end{array}$

\section{Film 1 Bystander B}

Target-present

$\begin{array}{lcccc}\text { Target choices } & 2 & 6.5 & 2 & 11 \\ \text { Foil choices } & 7 & 22.5 & 8 & 44.5 \\ \text { False rejections } & 22 & 71 & 8 & 44.5 \\ \text { Total } & 31 & 100 & 18 & 100\end{array}$

Target-absent 
Chapter 2

$\begin{array}{lrrrr}\text { Foil choices } & 6 & 35.3 & 10 & 29.5 \\ \text { Total } & 17 & 100 & 34 & 100\end{array}$

\section{Film 2 Thief}

Target-present

$\begin{array}{lcccc}\text { Target choices } & 7 & 35 & 14 & 45.1 \\ \text { Foil choices } & 2 & 10 & 2 & 6.5 \\ \text { False rejections } & 11 & 55 & 15 & 48.4 \\ & 20 & 100 & 31 & 100\end{array}$

Target-absent

$\begin{array}{lcccc}\text { Correct rejections } & 21 & 67.7 & 15 & 79.9 \\ \text { Foil choices } & 10 & 32.3 & 4 & 21.1 \\ \text { Total } & 31 & 100 & 19 & 100\end{array}$

\section{Film 2 Victim}

Target-present

$\begin{array}{lcccc}\text { Target choices } & 10 & 66.7 & 24 & 70.6 \\ \text { Foil choices } & 1 & 6.6 & 3 & 8.8 \\ \text { False rejections } & 4 & 26.7 & 7 & 20.6 \\ \text { Tal } & 15 & 100 & 34 & 100\end{array}$

\section{Target-absent}

$\begin{array}{lcccc}\text { Correct rejections } & 26 & 76.5 & 13 & 72.2 \\ \text { Foil choices } & 8 & 23.5 & 5 & 27.8 \\ \text { tal } & 34 & 100 & 18 & 100\end{array}$

\section{Film 2 Bystander}

Target-present

$\begin{array}{lcccc}\text { Target choices } & 18 & 53 & 11 & 61.2 \\ \text { Foil choices } & 6 & 17.6 & 5 & 27.7 \\ \text { False rejections } & 10 & 29.4 & 2 & 11.1 \\ \text { otal } & 34 & 100 & 18 & 100\end{array}$

Target-absent

$\begin{array}{lcccc}\text { Correct rejections } & 6 & 35.2 & 13 & 40.6 \\ \text { Foil choices } & 11 & 66.8 & 19 & 59.4 \\ \text { tal } & 17 & 100 & 32 & 100\end{array}$


Sensitivity and response bias. In light of the unusual way testing optimality affected choosing rates in target-present lineups, I drew from the signal detection theory approach (Green \& Sweets, 1966) to further clarify the observed effects. Signal detection theory is widely used in the recognition literature to understand the mechanisms behind distinguishing novel items from those that were encountered before. For this purpose, signal detection analyses isolate hit and false alarm rates to compute two independent factors that affect recognition performance. Discrimination accuracy $\left(d^{\prime}\right)$ is a measure that can be used to distinguish signals (in identification task signal refers to responses to targets) from noise (responses to foils). A value of zero indicates zero ability to distinguish targets from non-targets. Response bias $(c)$, on the other hand, refers to the threshold for deciding that participants have seen the target before. In the context of eyewitness identification, a negative $c$ value indicates a bias towards making a selection from a lineup, whilst a positive $c$ value shows a bias towards rejecting the lineup.

I tested the two $d^{\prime}$ and $c$ values for optimal versus non-optimal conditions collapsed across all lineups. Discriminability was slightly, though non-significantly higher in non-optimal, $d^{\prime}{ }_{\text {Non-Opt }}=1.72$, compared to optimal condition, $d^{\prime}{ }^{\prime} \mathrm{pt}=1.34, G=0.11, p=.915$. Response bias was $C_{\text {Non-Opt }}=1.09$ in the non-optimal sessions, compared to copt $=0.94$ in the optimal session. I am unaware of a significance test for the $c$ values, but on a descriptive level, the values do not to show a substantial difference between response bias levels in the two experimental conditions.

Figure 1 displays the ROC curves for optimal versus non-optimal testing conditions, with the diagonal line representing chance performance. The $x$ axis represents false alarm rates computed as the proportion of foil identifications in target-absent lineups divided by six (i.e., the lineup size; Brewer \& Wells, 2006), whereas the $y$ axis represents the proportions of target selections in target-present lineups. Due to few observations in levels 0 to $40 \%$, I collapsed these categories. The comparison of the curves confirms results of the GEE analyses: The curves shows higher hit rates in non-optimal compared to optimal sessions at all the cut-off levels, whereas the false alarm rates were similar for the two conditions. 


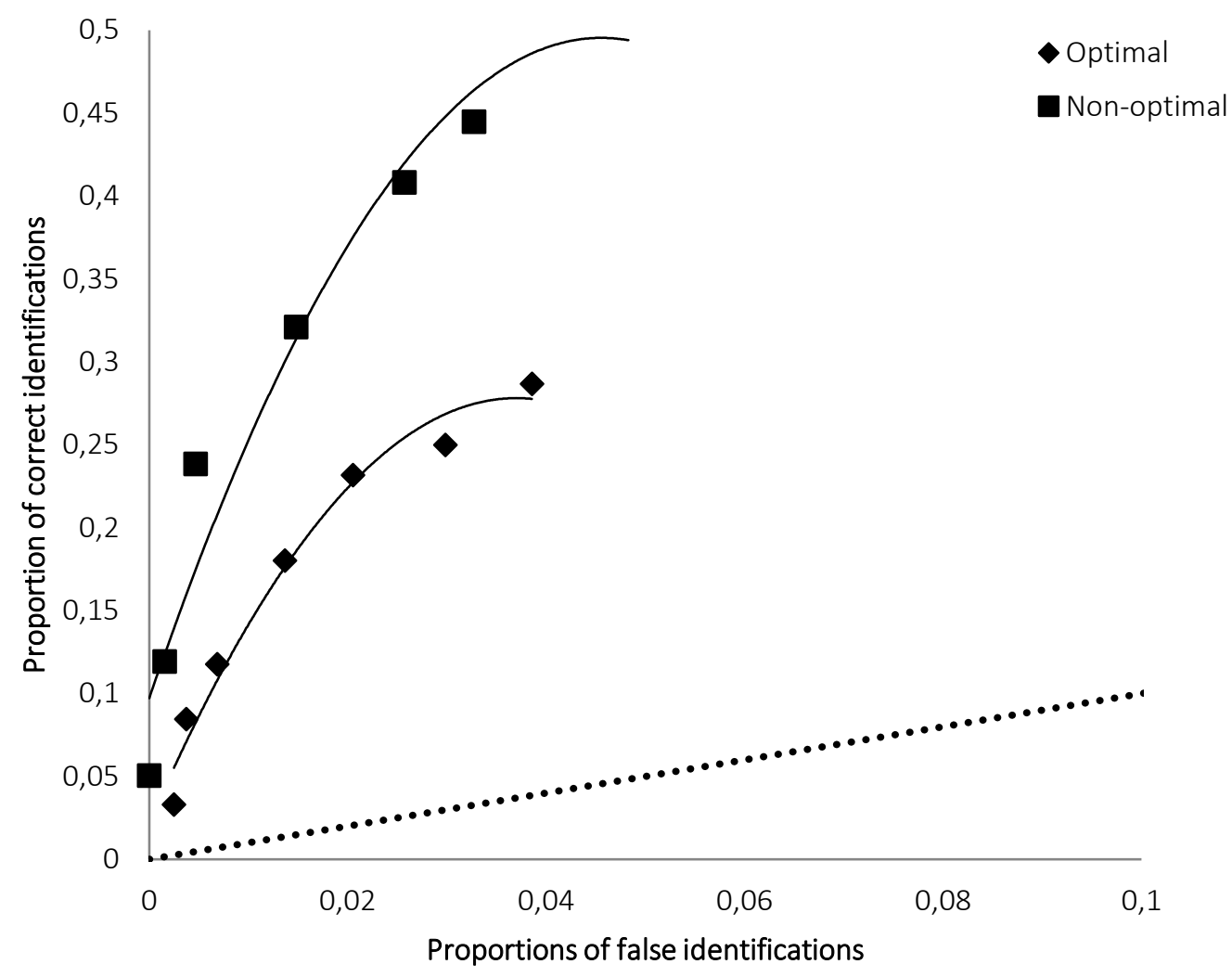

Figure 1. Receiver operating characteristics plots for the optimal and the non-optimal testing sessions across all lineups in Experiment 1.

Effect of time-of-day optimality on the confidence-accuracy relationship. To establish the effect of time-of-day optimality on the confidence-accuracy relationship, I entered identification accuracy (accurate versus inaccurate), choosing (selection versus rejection), and time-of-day optimality (optimal versus non-optimal), as well as their two- and three-way interactions as predictors in the GEE model. Non-significant terms were deleted stepwise. The final model contained only the significant Identification Accuracy $x$ Choosing interaction, Wald $\chi^{2}(1)=13.80, p<.001$. Simple slopes analysis showed that accurate choosers were more confident than inaccurate choosers, $\operatorname{Exp}(B)=3.09$, Wald $\chi^{2}(1)$ $=50.40, p<.001$, whereas this was not the case for nonchoosers, $\operatorname{Exp}(B)=.82$, Wald $\chi^{2}(1)=1.09, p=$ .297. These results replicate the common finding that accurate selections are made with more confidence than inaccurate selections, whereas no such relationship is observed in rejections (Sporer et al., 1995; Weber \& Brewer, 2003, 2004). However, contrary to my expectations, this interaction effect was not moderated by testing optimality.

Calibration of confidence measures. Calibration analyses can be an informative way to explore the association between the objective accuracy probabilities and subjective post-decision confidence measurements. There are several ways to assess how well eyewitnesses are calibrated in their confidence judgements. First, the proportion of accurate decisions for each confidence level can be plotted against the mean confidence for the respective level to create a calibration curve. Visual 
inspection of the curve allows to assess how well-calibrated participants were in each of the levels of confidence. Second, the calibration statistic (C) provides a quantitative reflection of the level of deviation from perfect calibration. It ranges from 0 (perfect calibration) to 1 (poorest calibration). Third, the over/underconfidence $(\mathrm{O} / \mathrm{U})$ statistic is a further indicator of how well-calibrated participants are. It varies from -1 to +1 , with negative scores reflecting underconfidence and positive scores showing overconfidence. Finally, the normalized resolution index (NRI) allows to evaluate how good participant's confidence judgments are at discriminating accurate from inaccurate decisions. It ranges from 0 (lowest resolution possible) to 1 (perfect discrimination; Weber \& Brewer, 2004).

As expected and confirming the GEE analyses, the confidence-accuracy correlation was significant for choosers, $r(380)=.36, p<.001$, but not for nonchoosers, $r(319)=.04, p=.478$. Note the (nearly) large effect size for choosers that corresponds well with the effect reported in the meta-analysis (Sporer et al., 1995). Following Flowe et al. (2017), I collapsed the confidence categories into three categories (i.e., 0-40\%; 50-70\%; 80-100\%) to provide more stable estimates for each confidence category. The proportion of accurate decisions for each of the collapsed categories was plotted against the weighted mean confidence for that category to create the confidence-accuracy calibration curve. 

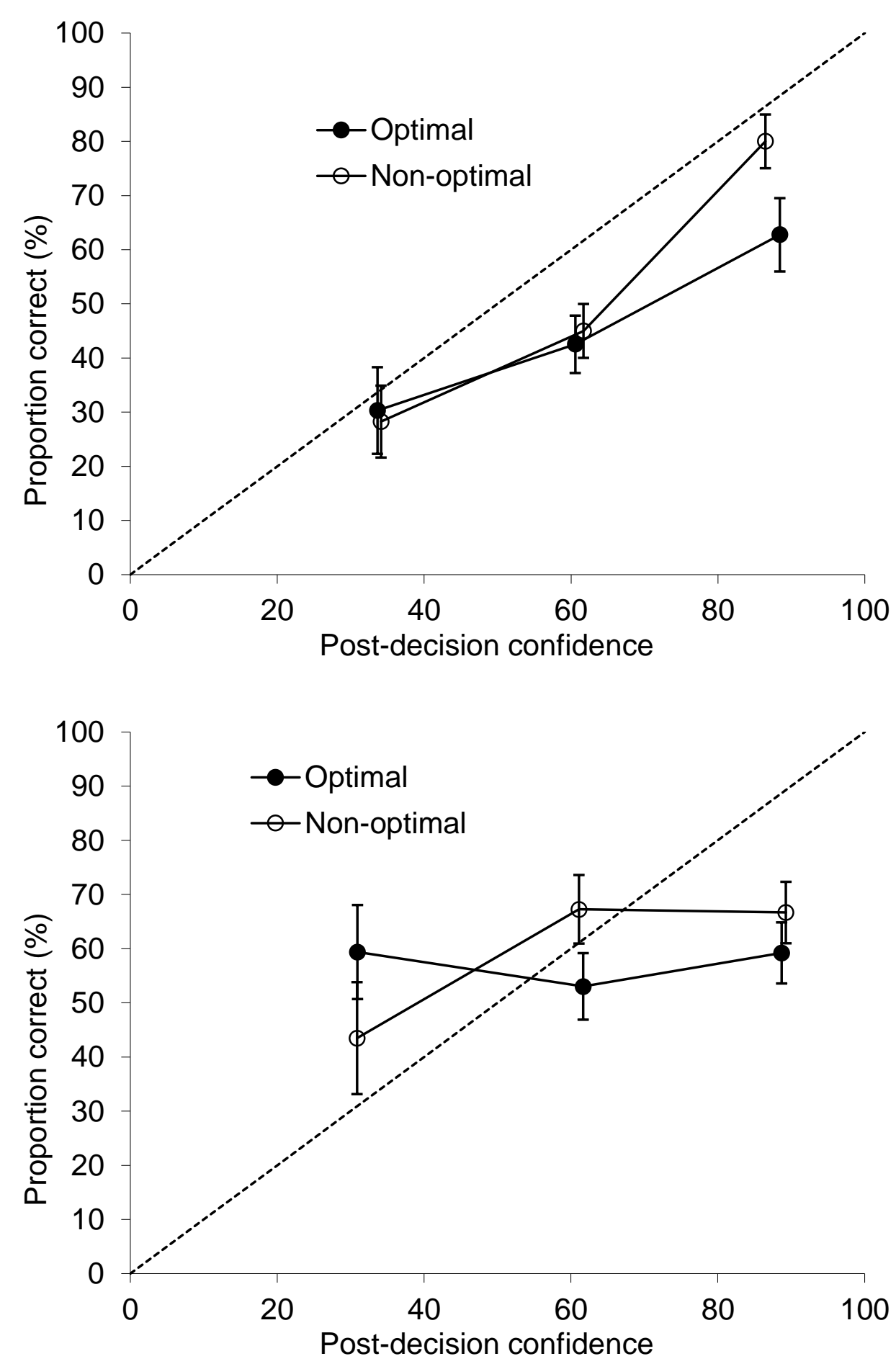

Figure 2. Post-decision confidence-identification accuracy calibration curves (and $95 \% \mathrm{Cl}$ bars) for the optimal and the non-optimal time-of-day in choosers (top panel) and nonchoosers (lower panel) in Experiment 1. 
The top panel of Figure 2 displays the confidence-accuracy calibration curves for choosers at the optimal (number of observations $n=171$ ) and the non-optimal ( $n=211$ ) time of day. It shows that the curve generally follows the identity line. The comparison of confidence intervals reveals that calibration in the last category (80-100\%) was significantly better at the non-optimal compared to the optimal time of day. The lower panel of Figure 2 shows the confidence-accuracy calibration curves for nonchoosers at the optimal $(N=174)$ and the non-optimal $(N=147)$ time of day. The calibration curves for non-choosers almost parallel the X-axis, though calibration in the second category (40-70\%) significantly differed at non-optimal as compared to the optimal time of day. Confidence intervals for the other confidence categories in choosers and nonchoosers overlap. Calibration statistics presented in Table 3 also show no significant differences between the optimality conditions.

Table 3

Calibration measures choosers and nonchoosers as a function of time of testing

\begin{tabular}{|c|c|c|c|c|}
\hline & \multicolumn{2}{|c|}{ Optimal } & \multicolumn{2}{|c|}{ Non-Optimal } \\
\hline & Measure & $95 \% \mathrm{Cl}$ & Measure & $95 \% \mathrm{Cl}$ \\
\hline \multicolumn{5}{|l|}{ Choosers } \\
\hline C & .013 & $.000 ; .026$ & .023 & $.004 ; .041$ \\
\hline$O / U$ & .059 & $.003 ; .116$ & .116 & $.055 ; .176$ \\
\hline$N R I$ & .066 & $.003 ; .129$ & .030 & $.022 ; .181$ \\
\hline \multicolumn{5}{|l|}{ Nonchoosers } \\
\hline C & .031 & $.008 ; .054$ & .031 & $.005 ; .057$ \\
\hline$O / U$ & -.058 & $-.124 ; .007$ & -.008 & $-.086 ; .069$ \\
\hline$N R I$ & .019 & $-.017 ; .055$ & .004 & $-.014 ; .022$ \\
\hline
\end{tabular}

Confirming GEE analysis, these findings show no support for my hypothesis that postdictive value of confidence would be stronger at the optimal time of day. In fact, the calibration analyses show some evidence in the opposite direction: Participants were better calibrated in some of the confidence categories in the non-optimal compared to the optimal sessions.

Effect of time-of-day optimality on the decision time-accuracy relationship. The decision time distributions showed significant positive skewness and kurtosis. Therefore, I conducted the analyses on log-transformed data (log base 10). To establish the effect of time-of-day optimality on the decisiontime-accuracy relationship, I entered identification accuracy (accurate versus inaccurate), choosing (selection versus rejection), time-of-day optimality (optimal versus non-optimal), their two-way and three-way interactions as predictors in the GEE model. Log-transformed decision times served as the dependent variable. The model revealed a significant three-way interaction, Wald $\chi^{2}(1)=4.03, p=.045$. Simple effects analyses of the Accuracy $x$ Choosing interaction revealed a significant interaction effect at the optimal, Wald $\chi^{2}(1)=6.19, p=.013$, but not at non-optimal time-of-day, Wald $\chi^{2}(1)<0.01, p=.954$. 
Further analyses were performed on choosers and nonchoosers that were tested at the optimal timeof-day. The simple simple effect of accuracy was not significant for choosers, $b=0.07$, Wald $\chi^{2}(1)=3.31$, $p=.069$, or nonchoosers, $b=-0.07$, Wald $\chi^{2}(1)=3.60, p=.058$. These results confirm my hypothesis, showing that non-optimal testing eliminated the postdictive value of decision times. However, the simple simple effect analyses failed to show a common finding that accurate chooser decisions were faster than inaccurate chooser decisions (Brewer \& Wells, 2006; Dunning \& Stern, 1994; Sauerland \& Sporer, 2007, 2009).

Effect of time-of-day optimality on memory performance in the DRM paradigm. I conducted oneway repeated measures ANOVAs to determine whether testing optimality led to significant differences in proportions of yes responses to presented items, critical lures and non-presented non-related items. Recognition of presented items did not significantly differ between the optimal $(M=.77, S D=.11)$ and non-optimal $(M=.75, S D=.11)$ conditions, $F(1,99)=1.50, p=.223, \eta_{p}{ }^{2}=.02$. Similarly, proportions of Yes responses to critical lures were not affected by testing optimality, $F(1,99)=0.05, p=.822, \eta_{p}{ }^{2}=.01$, with $M_{o p t}=.32, S D_{o p t}=.16$ and $M_{\text {Non-opt }}=.32, S D_{\text {Non-opt }}=.17$. Finally, response rates to non-presented unrelated items did not significantly differ between the optimal $(M=.08, S D=.08)$ and the non-optimal $(M=.08, S D=.08)$ time of day, $F(1,99)=0.03, p=.8, \eta_{p}{ }^{2}<.01$. Net accuracy (calculated as the ratio of hit rates to hit rates plus false alarms to unrelated foils) did not significantly differ between the optimality conditions, $F(1,99)<0.01, p=.979, \eta_{p}^{2}<.01$.

Due to potential 'yea-saying' bias, it is common to transform hit and false alarm rates using the two-high-threshold correction, in which the proportion of yes responses to non-presented unrelated items is subtracted from hit rates and false alarms to critical lures (Otgaar et al., 2014; Snodgrass \& Corwin, 1988). After I applied the correction, the pattern of findings was similar to the one obtained with untransformed scores. That is, testing optimality affected neither recognition of presented items, $F(1$, $99)=0.91, p=.342, \eta_{p}{ }^{2}=.01\left(M_{o p t}=.69, S D_{o p t}=.14\right.$ and $\left.M_{\text {Non-opt }}=.67, S D_{\text {Non-opt }}=.14\right)$, nor responses to critical lures, $F(1,99)=0.11, p=.746, \eta_{p}^{2}=.02\left(M_{o p t}=.24, S D_{\text {opt }}=.15\right.$ and $\left.M_{\text {Non-opt }}=.24, S D_{\text {Non-opt }}=.17\right)$. I also obtained similar results when examining whether optimality affected performance for each scene separately ( $p s>$.05). Table 4 presents the mean hit rates, false alarms to critical lures and unrelated foils, and net accuracy across the four pictures. 
Table 4

Proportions of Yes responses to presented items (hit rates), critical lures (false recognition) and non-presented non-related controls at the optimal and the non-optimal time of day

\begin{tabular}{|c|c|c|c|c|c|c|c|c|c|c|}
\hline \multirow[b]{3}{*}{ Scene } & \multicolumn{10}{|c|}{ Time of testing } \\
\hline & \multicolumn{5}{|c|}{ Non-optimal } & \multicolumn{5}{|c|}{ Optimal } \\
\hline & Positive & Neutral & Negative & $\begin{array}{l}\text { Delusional } \\
\text { paranoid }\end{array}$ & Total & Positive & Neutral & Negative & $\begin{array}{l}\text { Delusional } \\
\text { paranoid }\end{array}$ & Total \\
\hline Hit rate & .72 & .69 & .82 & .77 & .72 & .73 & .71 & .84 & .81 & .73 \\
\hline Hit rate* & .47 & .67 & .80 & .73 & .47 & .49 & .68 & .82 & .77 & .49 \\
\hline False recognition & .39 & .33 & .33 & .26 & .39 & .37 & .32 & .32 & .29 & .37 \\
\hline False recognition * & .14 & .31 & .30 & .23 & .14 & .13 & .29 & .30 & .26 & .13 \\
\hline Non-related & .25 & .02 & .03 & .04 & .25 & .24 & .02 & .02 & .03 & .24 \\
\hline Net Accuracy & .67 & .70 & .74 & .76 & .67 & .68 & .71 & .75 & .75 & .68 \\
\hline
\end{tabular}

Note. ${ }^{*}$ corrected for response bias using two-high-threshold correction 


\section{Discussion}

The current experiment investigated the effect of chronotype synchrony on eyewitness performance. To the best of my knowledge, this is the first attempt to investigate this effect in this context. Based on the previous findings that show quite consistent circadian variations in cognitive performance (Schmidt et al., 2007), I expected optimal testing to facilitate memory performance across an array of memory measures relevant to eyewitness domain, including recall, lineup identification and production of spontaneous false memories. Overall, my results showed that previously reported findings on superior memory performance during circadian peaks do not translate into the eyewitness memory domain in the straightforward manner I anticipated.

For recall performance, I expected participants to provide a larger number and proportion of accurate details at the optimal compared to the non-optimal time of day. Contrary to my expectations, accuracy rates did not significantly differ across the experimental conditions. In fact, accuracy rates in cued recall tasks were high at both optimal and the non-optimal time of day. In this respect, it is worthwhile noting that apart from the experimental manipulation, all other encoding and retrieval conditions were optimal. Participants were asked to pay close attention to the stimuli and were warned that they would have to act as eyewitnesses. Recall performance was measured immediately after participants encoded the stimulus event. I used recall instructions that discouraged guessing. In this experiment, I decided in favour of examining the mere effect of circadian arousal in the absence of other impairing factors to exclude the possibility of unpredicted interactions with the main manipulation. Future research might investigate whether time-of-day optimality effects in recall of eyewitnesses emerge with the use of more difficult cued questions or in the presence of additional suboptimal optimal factors, e.g., an increased retention interval.

With regard to identification accuracy, I expected participants to make more accurate identification decisions at the optimal compared to the non-optimal time of day. Contrary to my expectations, the results demonstrated the opposite effect: Participants were more likely to make an accurate identification decision at their non-optimal compared to the optimal time of day. The effect was specific to target-present lineups; performance in target-absent lineups did not vary as a function of time of day. These findings run counter to the typical circadian patterns described in prior research: Typically, performance is better at the optimal compared to the non-optimal time of day (e.g., IntonsPeterson et al., 1999; May et al., 1993; May \& Hasher, 1998; Ryan, Hatfield, \& Hofstetter, 2002).

Additional analyses explored whether the participants were less accurate at the optimal time of day because they selected fillers more often (i.e., due to the increase in false alarms), or because they incorrectly rejected the lineups even though the target was there (i.e., due to reduction in hit rates). I found that foil identifications were not affected by testing optimality. Instead, the drop in accuracy at the optimal time of day was the result of increased rejections of target-present lineups. The observed effect size was small and it is unclear why the change in target selections was not accompanied by an effect in false alarms, as it is commonly observed in recognition memory performance studies (Wixted 
\& Mickes, 2014). I am also not aware of any other research that showed a decrease in hit rates during the circadian peak.

These findings suggest the possibility of a change in response criteria across the optimality conditions, which may be associated with the use of the repeated-measures design. Even though I used a cover story to distract participant's attention from the main interest of my experiment, it was not possible to 'blind' participants to the hour of the day when the testing occurred. Due to the fact that testing sessions took place twice at unusual hours of the day, participants may have become aware of the optimality manipulation, which may have potentially resulted in strategies aimed at compensating for the anticipated cognitive impairments in the non-optimal session, such as using a more conservative response criterion.

To explore this possibility, I used signal detection analyses to calculate response bias across the optimality conditions. Results showed that the difference between $c$ values across testing times was negligible. Notably, the existing adaptations of the SDT approach to identification data treat target selections as hits and selections from target-absent lineps as false alarms. As my analyses showed that the unusual 'reverse synchrony' effect was observed in target-present lineups only and affected target selections as opposed to foils selections differentially, the criterion shift may not have been fully reflected in the signal detection calculations. I am unaware of an existing adaptation of the STD analyses to identification data that would enable to detect criterion shift in target-present and target-absent trials differentially, leaving open the possibility of the shift in response bias. Future research may preclude such a criterion shift by using a between-subjects design, which would reduce participants' awareness of the possible circadian-related impairments in performance in non-optimal sessions. Overall, my 'reserve synchrony' finding is unexpected and the observed effect size is small, which points out the need for replication.

I am also interested in whether testing optimality affected participants' post-decision confidence. My hypothesis was that optimal testing would strengthen the predictive value of confidence often found in choosers but not non-choosers. I replicated the common finding that accurate selections are made with more confidence than inaccurate selections, whereas no such pattern is observed in rejections (Sporer et al., 1995; Weber \& Brewer, 2003, 2004). However, contrary to my expectations, this interaction effect was not moderated by testing optimality. Calibration analyses confirmed that calibration statistic, over-under-confidence and resolution did not significantly differ between optimality conditions. Visual inspection of the calibration curves showed that in some of the confidence categories, calibration was significantly better at the non-optimal compared to the optimal time of day; specifically, for highly confident choosers. These findings are at odds with the optimality hypothesis (Deffenbacher, 1980), which predicts a higher postdictive value of confidence under optimal information-processing conditions. Rather, my data better fit an alternative theory-driven confidence judgments hypothesis, which suggests that non-optimal conditions may not necessarily undermine confidence-accuracy calibration in situations when participants are aware of the presence of a factor that negatively affects their memory (Palmer, Brewer, Weber, \& Nagesh, 2013). In the context of my experiment, this could 
mean that due to their explicit morning or evening preference, the participants may have been aware of the possible cognitive impairments during the circadian troughs and taken this information into account in their metamemory judgements. This would explain the fairly good calibration in both optimality conditions with some evidence for somewhat superior calibration at non-optimal rather than the optimal time of day.

I also investigated whether testing optimality affected decision-time-accuracy relationship. I expected non-optimal testing to attenuate the predictive value of response latencies in lineup identifications in such a way that the decision-time-accuracy relationship in choosers would be weaker at the non-optimal compared to the optimal time of day. My results revealed that accurate choosers made faster decisions than inaccurate choosers, replicating a common pattern of results (Brewer \& Wells, 2006; Dunning \& Stern, 1994; Sauerland \& Sporer, 2007, 2009). The analyses also supported my prediction that optimality would act as a moderator of this effect: Non-optimal testing eliminated the postdictive value of decision times. These findings suggest that the previously shown decrease of the speed of accessing information from long-term memory at the non-optimal time of day (Anderson et al., 1991) makes the difference in decision times between accurate and inaccurate choosers undetectable. If replicated, these findings would suggest that time of day when identification procedures are performed could undermine the decision time-accuracy relationship in eyewitness identifications. These findings may also be of interest to memory researchers who rely on decision times as their outcome measure: It may be important to take into account that time of the day when the test is administered may confound the decision-time-based outcomes.

I was also interested in the effect of time-of-day optimality on the production of spontaneous false memories induced by the DRM paradigm. Results showed that testing optimality did not significantly affect hit rates or false alarms to critical lures. These findings are in line with earlier reports of higher false memory rates during circadian troughs in older but not younger adults (Intons-Peterson et al., 1999). However, in this experiment, age and chronotype were confounded (i.e., all younger participants were evening types). Therefore, my experiment extends previous knowledge on circadian variations in the production of spontaneous false memories by replicating this effect in sample consisting of both morning- and evening-type young adults. Combined with the data reported by Intons-Peterson et al. (1999), my results suggest that non-optimal testing does not produce significant differences in the production of spontaneous false memories in healthy young adults. Future research can focus on possible time-of-day variations in false memory rates under suboptimal conditions, such as divided attention or prolonged retention interval.

One limitation of my study concerns that encoding and retrieval took place in the same experimental session. This design did not allow us to assess the effects of non-optimal testing on encoding and retrieval differentially. Future studies may address this issue by separating the two memory stages into different testing sessions and manipulating testing optimality for each of them separately, for example by employing a testing optimality (optimal versus non-optimal) x memory stage (encoding versus retrieval) design. Additionally, the synchrony effect model I used does not allow to 
disentangle the effects of circadian rhythm from sleep-related factors that could potentially mask circadian fluctuations in alertness and performance. Future research can employ the so-called forced desynchrony protocol to obtain a clearer picture of the interrelations of the circadian and sleep-related contributions to memory performance in eyewitnesses. The forced desynchrony protocol allows to study the circadian rhythm by isolating participants from external time cues and imposing a 'day' with a duration other than 24 hours (e.g., 28 hours), allowing extra control over factors that can potentially confound the effects of circadian variations in alertness (Wright, Hull, Hughes, Ronda, \& Czeisler, 2006).

To conclude, creating optimal retrieval conditions for eyewitnesses is important for reducing identification errors and the associated potential of miscarriages of justice. This fact necessitates a closer examination of factors that contribute to memory performance in eyewitnesses. The current experiment provides an initial understanding of the role of one of such factors, namely testing optimality, in eyewitness identification performance. My results suggest that the effect of testing optimality on decision-making processes of eyewitnesses manifests itself in a more complex way than previously shown with non-eyewitness-identification paradigms. This raises the question of generalizability of findings obtained basic experiments conducted in laboratory to applied settings such as the eyewitness memory domain. Future research can further elucidate this issue by combining basic recall and recognition tasks and eyewitness memory paradigm, which would allow to compare the differences in performance across the two types of memory tasks. 
Chapter 2 
CHAPTER 3

\section{Disentangling Chronotype and Time-of-day Effects on \\ Eyewitness Identification Accuracy}

Yaremenko, S., Sauerland, M., Hope, L. (2020) Circadian variations in eyewitness identifications:

Identification performance is not affected by time-of-day optimality. Manuscript submitted for publication, revisions invited. 


\begin{abstract}
Our body clock generates 24-hour cycles in alertness and affects our memory performance throughout the day. Individuals can differ in the hours of the day when their memory functions best: Morning types show highest recall and recognition during the morning hours, whereas evening types are at their best in the evening. In this experiment, I investigated the possibility that our body clock affects eyewitness identification outcomes. A total of 324 morning- and evening-type participants were recruited using the Amazon Mechanical Turk platform. Participants were randomly assigned to be tested in the morning (7:30 AM - 9:00 AM) or in the evening (8:30 PM - 10:00 PM). That is, each participant was tested either at their optimal or the non-optimal time of day. Surprisingly, results showed that identification accuracy depended on chronotype alone, irrespective of time of testing. Across all three lineups, evening types were 1.52 times more likely to make accurate identifications compared to morning types. Contrary to my expectations, confidence-accuracy relationship was stronger at the nonoptimal compared to the optimal time of day. I did not observe a decision-time-accuracy relationship, possibly due to reduced control over the experimental setting in an online environment. I discuss the peculiarities of encoding and retrieval conditions in eyewitnesses that could account for the discrepancy of my findings with basic experiments conducted in the psychological laboratory.
\end{abstract}




\section{Introduction}

Daily variations in our physiology, behaviour and cognition are inherent in our daily lives. These changes do not happen chaotically. Instead, a central 'pacemaker' of our body, known as the circadian clock (Halberg et al., 2003) ensures that the numerous systems in our body function in synchrony with each other and with the external environment. Located in the suprachiasmatic nucleus of hypothalamus, the circadian clock maintains 24-hour cycles in heart rate and body temperature, hormone levels and metabolism, sleep propensity and alertness levels (Czeisler \& Gooley, 2007; Fisk et al., 2018).

To a large extent, it is by virtue of the circadian clock that we experience peaks and dips in alertness and performance, as some hours of the day are more optimal for engaging in cognitively demanding activities than others (Krishnan \& Lyons, 2015; Kyriacou \& Hastings, 2010). In a phenomenon known as the synchrony effect, performance is better whenever the timing of the task is congruent with the circadian phase, and the other way around, the hours of the day that are incongruent with our internal body clock are associated with lowest performance (Schmidt et al., 2007).

Importantly, the circadian clock in some individuals is 'set' in a slightly different way than in others. The so-called morning types, also known as larks, who wake up and go to sleep earlier than others and prefer to be more active in the morning hours. Evening types, or owls, on the contrary, prefer to wake up and go to sleep later, and are at their best later in the evening than most people (Horne \& Östberg, 1976). This time-of-day preference is also referred to in the literature as the circadian typology, or chronotype (Roenneberg, Wirz-Justice, \& Merrow, 2003). A relatively large proportion of the adult population (about 40\%) belong to either a morning- or an evening-chronotype group (Adan et al., 2012).

Inter-individual differences in the functioning of the body clock can be an important factor determining the time of day that is most optimal in terms of cognitive performance. Across a wide range of cognitive domains, such as attentional capacities, working and long-term memory, inhibition of irrelevant responses, and avoidance of stereotype-based responses, morning types perform better in the morning, and, the other way around, evening types are better in the evening (e.g., Goldstein, Hahn, Hasher, Wiprzycka, \& Zelazo, 2007; May 1999; Nowack \& Van Der Meer, 2018). Likewise, episodic memory is subject to the synchrony effect: Experimental research to date suggests that recall and recognition performance is better at circadian peaks as opposed to circadian troughs (May, Hasher, \& Stoltzfus, 1993; Ryan, Hatfield, \& Hofstetter, 2002; Petros, Beckwith, \& Anderson, 1990; Yoon, 1997).

Prior research showing the synchrony effect in the functioning of our memory begs the question of whether time of day may also contribute to memory performance in applied settings, such as the eyewitness memory domain. If time of day determines information processing efficiency, could eyewitnesses be better at identifying the culprit from the crime they witnessed at their optimal as opposed to the non-optimal time of day? Or perhaps the hour of the day when the lineup is administered could affect identification outcomes? To the best of my knowledge, no published studies investigated these possibilities. In the light of such limited literature, in Chapter 2, I reported the first study to test possible time-of-day fluctuations in eyewitness identification performance. The results were surprising: In target-present lineups, participants were more accurate in the non-optimal compared to the optimal 
sessions. The size of this effect was small and its direction contrasted previous literature. Additionally, the increase of hit rates was not associated with an increase in false alarms, as it is usually observed in recognition literature (Wixted \& Mickes, 2014). Taken together, these issues highlighted the need for replication.

Thus, the current experiment was conducted to further investigate the effect of time-of-day optimality on eyewitness identification performance. I made several changes to the experimental procedure used in Chapter 2. I recruited participants via Amazon Mechanical Turk (MTurk), which allowed us to collect a more demographically diverse sample. The increased statistical power resulting from a larger sample (cf. Chapter 2) made it possible to explore the possibility that time-of-day optimality manifests itself differently in individuals with morning as opposed to evening chronotype. Furthermore, I gave preference to a between-subjects design in the current experiment as opposed to within-subjects optimality manipulation in Chapter 2. I expected that this design would allow us to mask the main hypothesis of the study more successfully, eliminate the possibility of learning effects, and exclude random variations in performance associated with the use of two stimulus events.

Time-of-day optimality can also decrease the ratio of conscious, controlled memory processes (e.g., recollection) in relation to unconscious, automatic memory processes (e.g., priming; May, Hasher, \& Foong, 2005). Specifically, controlled memory processes are subject to variability across the day in line with fluctuations in circadian arousal, whereas automatic memory processes appear to be unaffected by the circadian rhythm (Puttaert, Adam, \& Peigneux, 2018; Yang, Hasher, \& Wilson, 2007). As a result, the moments of the day with lowest circadian arousal may be characterized by increased reliance on automatic as opposed to controlled processes, making us prone to memory biases. I was interested in testing the hypothesis that, if present in the lineup administration procedure, factors that are known to bias eyewitness's memory would be magnified by non-optimal testing. As means of introducing bias, I used the so-called mug shot exposure effect. Prior research showed that exposing eyewitnesses to mug shots before performing lineup identification may bias eyewitnesses' decisions. Under such conditions, eyewitnesses may base their decision on familiarity gained due to the mug shot presentation rather than on their memory from the actual event, thus increasing the likelihood of innocent suspect misidentifications (for a review, see Deffenbacher, Bornstein, \& Penrod, 2006). I hypothesised that such erroneous identifications due to mug shot exposure would be more likely to occur during circadian troughs compared to circadian peaks.

\section{Experiment 2}

\section{Method}

The experiment was pre-registered on the Open Science Framework. The registration form can be accessed at https://osf.io/kafe7/?view only=bc5dfe1248f749678adb0cd8388c8b36.

Sample size. Power analysis for a two-tailed binomial logistic regression with G*Power v3.1 returned a required sample size of 310 (Faul, Erdfelder, Buchner, \& Lang, 2009; Faul, Erdfelder, Lang, \& Buchner, 2007). To achieve equal distribution of participants across the four experimental conditions, I tested two extra participants, which resulted in the planned sample size of 312. I used the following 
parameters: $\mathrm{OR}=2.058 ; \operatorname{Pr}(\mathrm{Y}=1 \mid \mathrm{X}=1) ; \mathrm{HO}=0.383 ; \mathrm{R}^{2}$ other $\mathrm{X}=0.2, \mathrm{X}$ distribution = Binomial, $\mathrm{X}$ parm $\pi=$ 0.5. I based the odds ratio on the corrected recognition scores reported in a study by May et al. (1993). I used an alpha error probability of .05 and a power of .80 .

Participant pool. Participants were recruited with the help of Amazon Mechanical Turk (MTurk) platform. MTurk is an online crowd sourcing system that was developed with an aim to connect potential workers (MTurkers) with the so-called requesters, who offer jobs or tasks that can be completed online. Researchers have been increasingly using the MTurk platform for the purposes of data collection due to the multiple advantages it offers for academic research (Mason \& Suri, 2011; Paolacci \& Chandler, 2014). MTurkers do not appear to be statistically significant outliers in terms general demographics, and in some aspects the platform offers access to samples that are more representative compared to student samples (Buhrmester, Kwang, \& Gosling, 2011; Paolacci, Chandler, \& Ipeirotis, 2010). The psychometric properties of responses collected on MTurk have been validated, and multiple laboratories managed to replicate some of the classical findings using the platform (e.g., Crump, McDonnell \& Gureckis, 2013; Rand, 2012). Amazon MTurk offers opportunities for efficient and less costly data collection with reliable results. Combined with the fact that MTurkers are known to complete tasks around the clock, this has guided us in my decision to collect the data for this experiment using the platform.

Data validity checks and inclusion criteria. Online testing can increase the rates of careless or partially random responses (Meade \& Craig, 2012), an issue which may be of extra concern in studies that rely on Amazon MTurk platform in participant recruitment (e.g., Fleischer, Mead, \& Huang, 2015). Therefore, I took a series of additional measures to exclude random or careless responses and other problems related to the lack of experimental control in the MTurk environment.

Pre-screening. I included data quality check items in the pre-screening questionnaire, which allowed me to identify attempts of careless or automatic responses. One of the items paraphrased an MEQ question ("How difficult do you find it to get up in the morning (when you are not awakened unexpectedly)?" in the original version as opposed to "How easy do you find it to get up..." in the modified item). The other item duplicated an MEQ question ("At approximately what time of day do you usually feel your best?") in a form of a text entry question, i.e., participants were required to manually type in the time-of-day in AM or PM format. Each of the questions of the pre-screening survey was presented on a separate page, and participants could not go back to check their previous responses. Second, I relied on the duration of the response to the pre-screening questionnaire as an indicator for random or careless responses. I considered response duration below 2:30 min to be an indicator or careless or random responding for a survey containing 37-items. MTurkers who produced incorrect responses to the validity items or short response duration were not invited for participation.

Main experiment. Further data quality check items were included in the main part of the study. First, one of the MEQ items from the pre-screening questionnaire and three demographics questions were duplicated in the main experiment, allowing me to check for consistency of participant's response to these items across the two parts of the study. Second, I included the Instructional Manipulation Check (IMC; Oppenheimer, Meyvis, \& Davidenko, 2009) in the concluding phase of the main part of the study. 
The IMC includes a question in a form of a block of text with lure responses, overall mimicking a typical multiple choice question. However, the long text block contains the instruction to submit a counterintuitive response. The accurate response to IMC could serve as one of the indicators that participants have been following the study instructions carefully. Finally, at the end of the experiment, participants had to answer two simple questions related to the content of the stimulus film. Specifically, participants were asked about the item that was stolen in the incident (multiple choice) and were instructed to indicate what the thief did with the stolen item (free entry field). Thus, one of the control questions referred to the middle of the stimulus film, while the other one concerned the detail at the end of the film. This allowed me to detect careless or inattentive MTurkers and was also helpful in identifying cases when participants did not encode the stimulus fully due to some technical problems. Participant's response was counted as reliable only if they passed the IMC, showed consistent responses to the control items and responded to the questions related to the stimulus film accurately.

Possible character misrepresentation. Another potential problem is the possibility of character misrepresentation in Amazon MTurk samples. More specifically, experiments that target specific populations may encourage some MTurkers to falsify their identities (i.e., claim to belong to certain categories of population) or make multiple attempts to pass the screener questions to qualify for the main study and receive higher reimbursement. To address this concern, I adopted a two-step prescreening procedure to prevent MTurkers from falsifying the answers to the pre-screening questionnaire to qualify for the main experiment (Wessling, Huber, \& Netzer, 2017). Specifically, I aimed to create an impression that the pre-screening questionnaire was actually an independent survey focusing on the way sleep habits affect eating and caffeine consumption behaviour. There was no indication of a link between the pre-screening and the main study. The main study was visible only to MTurkers who were eligible and passed the initial data quality checks.

Participants. I pre-screened a total of 4,270 MTurkers. Among the pre-screened participants, 1,478 were morning-types and 568 were evening-types. A total of 365 proceeded to participate in the main study, of which 39 were excluded because they did not meet some of the data quality checks.

Upon completion of data collection, I discovered that due to an unanticipated technical error, the question asking participants to indicate their lineup decision appeared on a separate rather than the same page as the lineup itself for 12 of the tested participants. In the event that this formatting discrepancy produced any distortion in responses provided by these participants, I collected data for a further 12 participants ${ }^{3}$.

Hence, the final sample consisted of 324 participants (160 male, 163 female, 1 unspecified; age 19 to $66, M=35.6, M d n=34$ years $)$. A total of 118 of them showed evening preference $\left(36.4 \%, M_{M E Q}=\right.$

${ }^{3}$ Subsequently, I ran all analyses twice, once including the additionally collected 12 responses and once excluding them. The pattern of results was analogous. Therefore, in the results section I report statistical output for analyses performed including data collected from all participants. 
35.8, SDMEQ $=3.99)$, and 206 participants were morning types $\left(63.6 \%, M_{\mathrm{rMEQ}}=64.03, S D_{\mathrm{rMEQ}}=4.59\right)$. Participants received a $\$ 3$ honorarium on completion of the experiment.

Design. The study used a two-factorial between-participants design with time-of-day optimality (optimal versus non-optimal) and target presence (present versus absent) as independent variables. Participants were randomly assigned to be tested either at their optimal or the non-optimal time of day. I counterbalanced target presence of each lineup across the optimality conditions. Additionally, I partially counterbalanced the combinations of target presence in the three lineups: Participants received either one target-present and two target-absent lineups, or one target-absent and two target-present lineups.

In the thief lineups, I additionally manipulated mug shot exposure bias (bias versus no bias). In the biased condition, one individual appeared among both the mugshots and the lineup foils. However, only one participant in the biased condition selected the innocent suspect from the lineup, suggesting that the biasing manipulation was not successful.

Participants made three identification decisions. For each of the three lineups, target-presence was counterbalanced across the two optimality conditions. Identification accuracy (accurate versus inaccurate), post-decision confidence (on an 11-point scale ranging from 0-100\%) and decision times (in ms) served as dependent variables.

\section{Materials.}

Pre-screening questionnaire. I used the full version of the MEQ (Horne \& Östberg, 1976) to identify participants' time-of-day preference. The 19 original MEQ items were intermixed with filler questions related to participants' sleep, food and caffeine consumption habits to mask the aim of the pre-screening survey and provide additional support for the cover story. Example items are "How much control do you have over your eating between supper and bedtime?", "At what time do you prefer to drink your first caffeine-containing beverage?", "When you get up in the middle of the night, how often do you snack?" Additionally, the questionnaire included the two data quality check items.

Stimulus film. I used Film 2 from Experiment 1 as the stimulus event.

Mug books. I constructed two mug books, containing 16 head-and-shoulder photographs of white males that met the description of the thief from the stimulus film. The biased mug book contained a photograph of the innocent suspect; this person would also appear in the thief lineup as the incidental target. The unbiased mug book included a photograph of a control foil.

Photo lineups. I created six-person target-present and target-absent simultaneous lineups for each of the three targets appearing in the stimulus film, namely, the thief, the victim and the bystander. Target-absent lineups were constructed containing photographs of fillers that matched the description of the target in a similar way as in Experiment 1. I used a more thorough scheme of counterbalancing target positions and foil choices in the lineups compared to Experiment 1 to eliminate the possibility of obtaining a result that is attributable to some specifics of the retrieval stimuli. For target-present lineups, I created six versions of lineups, with all possible combinations of the target with five of the foils from the target-present lineup. The target positions in the thief, victim, and bystander lineup were 3, 6, and 3, respectively. The incidental target (i.e., the innocent suspect who appeared among the biased mug 
shots) took position 5 in the thief target-present (see Figure 3, left panel) and target-absent lineups. Additionally, I created six extra target-present thief lineups in which positions of the thief and the innocent suspect were interchanged, that is, the thief took position 5 and the innocent suspect position 3 (Figure 3, right panel). Positions of the foils were randomized for each of the lineup versions. This resulted in a total of 12 versions of thief-present lineups, six versions of victim and six versions of bystander lineups.

I established the effective lineup sizes in a pilot study. For each of the targets, I tested the target-absent lineup and one randomly selected version of the target-present lineup. Tredoux's Es ranged from 3.5 to 4.6 (19 to 20 mock witnesses, total $N=119$ ).
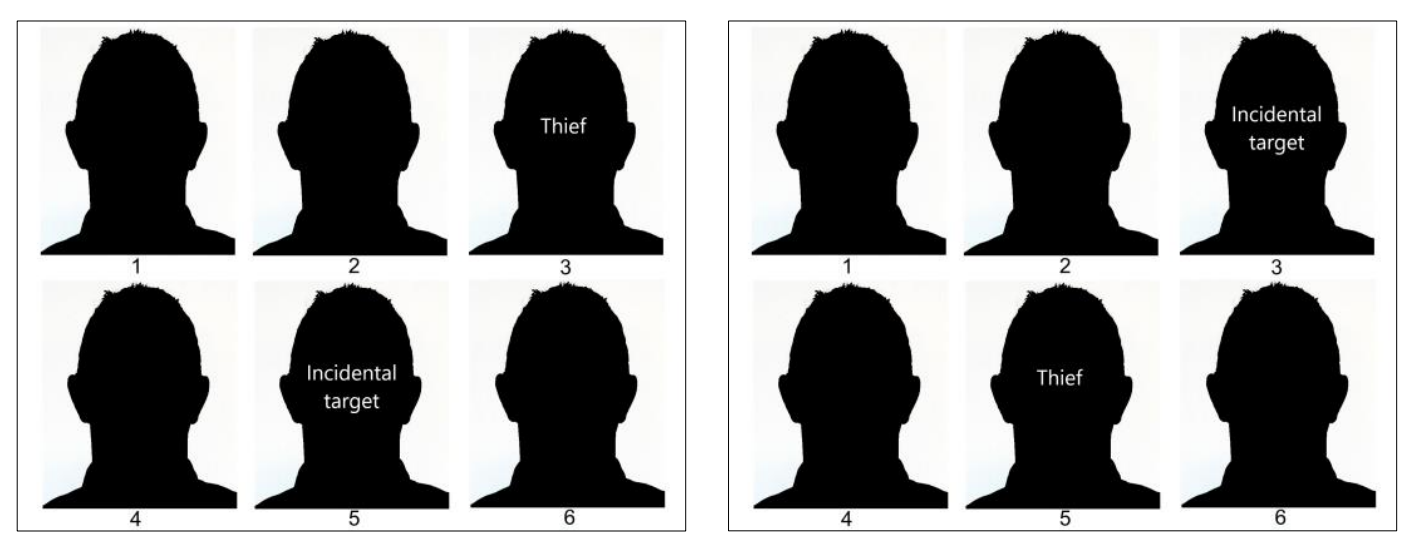

Figure 3. Positions of thief and incidental target in target-present biased perpetrator lineups.

Procedure. Participants who fulfilled the pre-screening criteria received access to the main study and a personal message inviting them for participation. As a cover story, I informed them that the experiment focused on long-term effects of eating, caffeine-consumption habits and circadian rhythms on memory performance. I instructed participants not to consume alcohol for 8 hours prior to testing, more than two cups of coffee on the day of testing and sleep a minimum of six hours in the night prior to testing to exclude the possibility of confounding effects on alertness and memory performance.

Testing sessions took place either between 7:30 AM and 9:00 AM or between 8:30 PM and 10:00 PM. I used more extreme early morning and late evening hours compared to Experiment 1 to maximize the possible synchrony effect. Participants watched a stimulus film and were instructed to watch the film closely and pay attention to every detail and that they will be asked to act as eyewitnesses. Then, they provided answers to seven multiple choice questions concerning their food consumption habits (e.g., "How hungry are you usually in the morning?"); these were included for additional support of the cover story. Next, participants viewed the mug book. When all 16 mug shots had been presented, participants could make a selection or press the "Not present" option. As filler tasks, participants engaged in stem completion tasks adopted from Jacoby (1998) and an object search filler task for about 20 minutes. The results concerning the effect of testing optimality on performance in the stemcompletion tasks are reported elsewhere. 
Following the filler tasks, participants viewed the three lineups in succession and attempted to identify the thief, the victim and the bystander who were involved in the stimulus event. The thief lineup always appeared first. The remaining two lineups were presented in a random order. Participants were informed that the targets may or may not be present in the lineups and were encouraged to select the "Not present" option if they were not sure or didn't know. After each of the decisions, participants indicated their confidence. Decision times for each identification decision were recorded automatically.

At the end of the experiment, participants were presented with a block data quality check items. The debriefing occurred upon completion of data collection.

\section{Results}

Data analyses. The pre-registered analysis plan was based on the assumption that presenting a mug shot of an innocent suspect in the biased conditions would affect the further identification decision in lineups. Because this manipulation was unsuccessful, my analyses deviated from the pre-registered analysis plan in two respects. First, I could not analyse the effects of my predictors on innocent suspect misidentifications. Second, I did not include the factor bias in my analyses of identification accuracy in the perpetrator lineups (i.e., I treated all identification decisions as unbiased) and hence conducted one analysis across all lineup decisions.

I was unable to verify whether 10 participants adhered to the requirements of participating within the specified timeframes. I re-ran all the analyses excluding these participants and obtained analogous results. Nonetheless, I excluded data from these participants from analyses due to importance of time of day in my experimental design.

Identification performance. Table 5 presents frequencies and proportions of different lineup outcomes across the experimental conditions. To ascertain the effects of time-of-day optimality and target-presence on the likelihood that participants made an accurate identification decision, I entered time-of-day optimality, target-presence, and their two-way interaction in a GEE model. I deleted nonsignificant terms stepwise. The final model only included the main effect of target presence: Participants had higher odds of making an accurate decision from target-absent compared to target-present lineups, Wald $\chi 2(1)=9.08, \operatorname{Exp}(B)=1.51, p=.002$. The effect of testing optimality did not materialize implying that I did not replicate the unusual findings obtained in Experiment 1, where performance in targetpresent lineups was better at the non-optimal compared to the optimal time of day. I also did not observe the synchrony effect previously reported in recognition performance of non-facial stimuli (IntonsPeterson, Rocchi, West, McLellan, \& Hackney, 1999; May et al., 1993). A Bayesian log-linear regression with time-of-day optimality, target-presence and accuracy in thief lineups, their two-way and three-way interactions as model factors showed strong evidence in support of null hypothesis, $\left(B F_{10}\right)=144.100$. 
Chapter 3

Table 5

Frequencies and proportions of different lineup outcomes at the optimal and the non-optimal time of day in Experiment 2

\begin{tabular}{lcccc}
\hline \multirow{2}{*}{ Lineup } & \multicolumn{2}{c}{ Optimal } & \multicolumn{2}{c}{ Non-optimal } \\
& Frequency & Proportion & Frequency & Proportion \\
\hline Thief & & & & \\
Target-present & & & 11 & $15.3 \%$ \\
Targets choices & 19 & $21.3 \%$ & 15 & $20.8 \%$ \\
Foil choices & 20 & $22.5 \%$ & 46 & $63.9 \%$ \\
False rejections & 50 & $56.2 \%$ & 72 & $100 \%$ \\
Total & 89 & $100 \%$ & & \\
Target-absent & & & & $64.7 \%$ \\
Correct rejections & 58 & $67.4 \%$ & 44 & $35.3 \%$ \\
Foil choices & 28 & $32.6 \%$ & 24 & $100 \%$ \\
Total & 86 & $100 \%$ & 68 &
\end{tabular}

Victim

Target-present

$\begin{array}{lllll}\text { Target choices } & 55 & 59.2 \% & 41 & 61.2 \% \\ \text { Foil choices } & 19 & 20.4 \% & 14 & 20.9 \% \\ \text { False rejections } & 19 & 20.4 \% & 12 & 17.9 \% \\ \text { otal } & 93 & 100 \% & 67 & 100 \%\end{array}$

Target-absent

$\begin{array}{ccccc}\text { Correct rejections } & 51 & 62.2 \% & 32 & 43.8 \% \\ \text { Foil choices } & 31 & 37.8 \% & 41 & 56.2 \% \\ \text { otal } & 82 & 100 \% & 73 & 100 \%\end{array}$

\section{Bystander}

Target-present

$\begin{array}{lcccc}\text { Target choices } & 45 & 54.8 \% & 41 & 56 \% \\ \text { Foil choices } & 19 & 23.2 \% & 16 & 22 \% \\ \text { False rejections } & 18 & 22 \% & 16 & 22 \% \\ \text { tal } & 82 & 100 \% & 73 & 100 \%\end{array}$


Target-absent

Correct rejections

Foil choices

Total
39

54

93
$41.9 \%$

$58.1 \%$

$100 \%$
27

40

67
$40.3 \%$

$59.7 \%$

$100 \%$

Differential effect of testing optimality in owls and larks. I ran an exploratory analysis to test the possibility that the effect of testing optimality on identification accuracy manifested itself differently in participants with morning and evening chronotype. I entered chronotype (morningness versus eveningness), test time (morning versus evening), target-presence (target-present versus target-absent), their two-way and three-way interactions as predictors into the model. Non-significant terms were excluded stepwise. The final model included the main effects of target presence, Wald $\chi^{2}(1)=9.62$, $\operatorname{Exp}(B)=1.51, p=.002$, and chronotype, Wald $\chi^{2}(1)=8.51, \operatorname{Exp}(B)=1.465, p=.004$. This means that participants had higher odds of making an accurate decision from target-absent compared to targetpresent lineups and that participants with evening preference were more likely to make an accurate identification decision than those with morning preference. The expected chronotype $\mathrm{x}$ test time interaction was not significant, indicating that the effect of chronotype occurred irrespective of the time of the day when testing occurred.

Sensitivity and response bias. Paralleling the signal detection analyses performed in Chapter 2, discriminability did not significantly differ between optimality conditions, with $d^{\prime}$ Non-opt $=1.18$ versus $d^{\prime} \mathrm{Opt}=1.41, G=0.66, p=.0 .94$. The response bias measures were again comparable for the two conditions, $c_{\text {Non-Opt }}=0.76 ;$ Copt $=0.79$.

Figure 4 displays the ROC curves for optimal versus non-optimal testing conditions, with the diagonal line representing chance performance. The two curves nearly overlap, confirming that there was no benefit from matching participant's time-of-day preference at any of the confidence cut-off levels. 


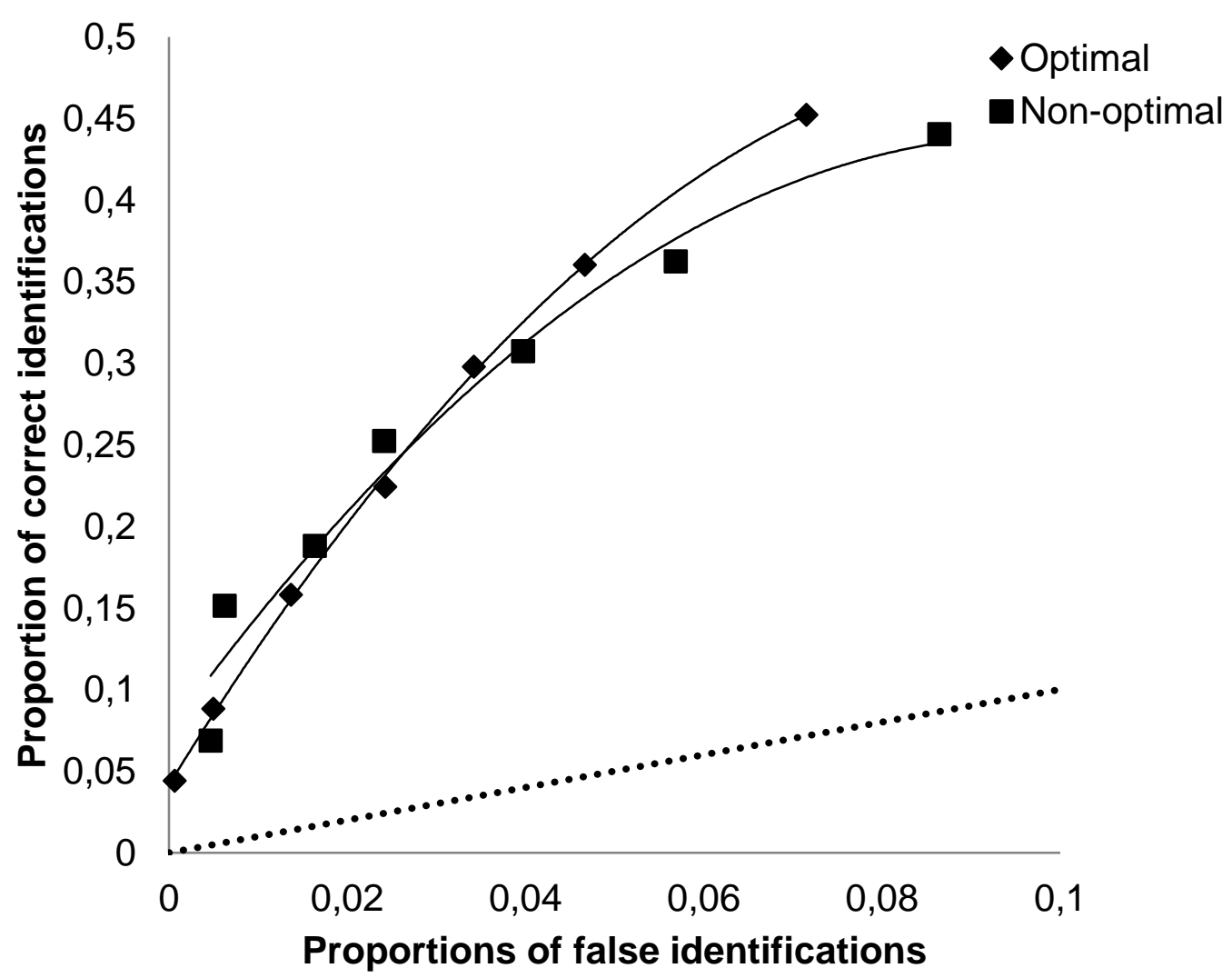

Figure 4. Receiver operating characteristics plots for the optimal and the non-optimal testing sessions across all lineups in Experiment 2.

Effect of time-of-day optimality on the confidence-accuracy relationship. To test the effect of time-of-day optimality on the confidence-accuracy relationship, I entered identification accuracy (accurate versus inaccurate), choosing (selection versus rejection), and time-of-day optimality (optimal versus non-optimal), as well as their two- and three-way interactions as predictors in the initial GEE model. The final model contained a significant Accuracy x Choosing x Optimality interaction, Wald $\chi^{2}(1)$ $=4.99, p=.026$. Separate analyses split by time-of-day optimality revealed a significant simple Accuracy $x$ Choosing interaction at non-optimal, Wald $\chi^{2}(1)=14.92, p<.001$, but not optimal time of day, Wald $\chi^{2}(1)=0.65, p=.420$. Further analyses performed on choosers and nonchoosers that were tested at nonoptimal time of day showed that accurate choosers were more confident than inaccurate choosers, $b=$ 1.36, Wald $\chi^{2}(1)=29.45, p<.001$. This effect was not statistically significant for nonchoosers, $b=0.16$, Wald $\chi^{2}(1)=0.69, p=.408$. Contrary to the findings reported in Chapter 2 , these results show that timeof-day optimality did affect the postdictive value of confidence. However, the direction of the effect was opposite than I hypothesized: The confidence-accuracy relationship in choosers was stronger at the nonoptimal compared to the optimal time of day.

Calibration of confidence measures. The confidence-accuracy correlation was significant for choosers, $r(527)=.27, p<.001$, and for nonchoosers, $r(414)=.11, p=.031$. Figure 2 (panel C) displays the confidence-accuracy calibration curves for choosers at optimal (number of observations $n=300$ ) and 

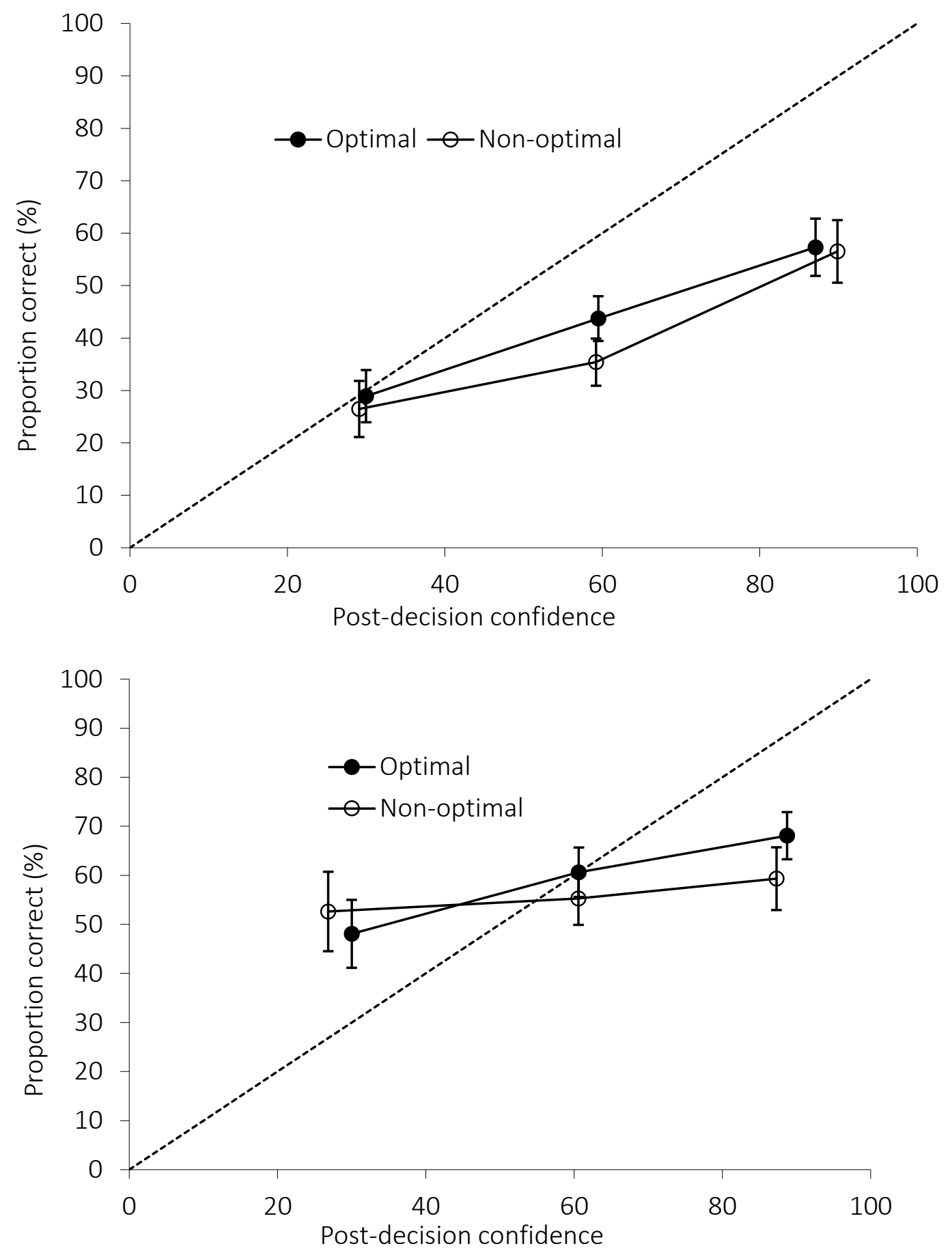

Figure 5. Post-decision confidence-identification accuracy calibration curves (and $95 \% \mathrm{Cl}$ bars) for the optimal and the non-optimal time-of-day in choosers (top panel) and nonchoosers (lower panel) in Experiment 2.

non-optimal ( $n=250$ ) time of day. Visual inspection of the curves reveals no significant differences between optimal and non-optimal testing (all the confidence intervals overlap). Similarly, nonchoosers did not show any significant differences between the two conditions: The calibration curves for both optimal $(n=240)$ and non-optimal $(n=182)$ sessions almost parallel the X-axis (see lower panel of Figure 
5). In line with findings obtained in Experiment 1, the confidence intervals for all calibration statistics overlap (see Table 6).

\begin{tabular}{|c|c|c|c|c|}
\hline \multicolumn{5}{|l|}{ Table 6} \\
\hline \multicolumn{5}{|c|}{ Calibration measures choosers and nonchoosers as a function of time of testing } \\
\hline & \multicolumn{2}{|c|}{ Optimal } & \multicolumn{2}{|c|}{ Non-Optimal } \\
\hline & Measure & $95 \% \mathrm{Cl}$ & Measure & $95 \% \mathrm{Cl}$ \\
\hline \multicolumn{5}{|l|}{ Choosers } \\
\hline C & .013 & $.000 ; .026$ & .023 & $.004 ; .041$ \\
\hline$O / U$ & .059 & $.003 ; .116$ & .116 & $.055 ; .176$ \\
\hline$N R I$ & .066 & $.003 ; .129$ & .030 & $.022 ; .181$ \\
\hline \multicolumn{5}{|l|}{ Nonchoosers } \\
\hline C & .031 & $.008 ; .054$ & .031 & $.005 ; .057$ \\
\hline$O / U$ & -.058 & $-.124 ; .007$ & -.008 & $-.086 ; .069$ \\
\hline$N R I$ & .019 & $-.017 ; .055$ & .004 & $-.014 ; .022$ \\
\hline
\end{tabular}

Effect of time-of-day optimality on the decision time-accuracy relationship. To establish the effect of time-of-day optimality on the decision-time-accuracy relationship, I entered identification accuracy (accurate versus inaccurate), choosing (selection versus rejection), time-of-day optimality (optimal versus non-optimal), their two- and three-way interactions in a GEE model. As in Experiment 1, I used log-transformed decision times (log base 10) as the dependent variable due to significant positive skewness and kurtosis. The final model contained only the main effect of Choosing, $b=.05$, Wald $\chi^{2}(1)=$ $10.99, p=.001$. Participants who made a selection were faster than those who rejected the lineup.

\section{Discussion}

Over the past decades, research has collected sufficient evidence showing that time of day can affect cognitive performance across an array of cognitive domains, which also include episodic memory. This guided my interest in investigating whether time of day is a factor of relevance for the eyewitness memory field, in particular with regards to eyewitness identification performance. I expected that testing participants at the optimal as opposed to the non-optimal time of day would have a beneficial effect on identification accuracy. However, in the current study optimal testing had no beneficial effect on identification accuracy. These findings do not parallel previously reported patterns in recognition memory, which is normally superior during circadian peaks as opposed to circadian troughs (IntonsPeterson et al., 1999; May et al., 1993; Petros et al., 1990). I also did not replicate the unusual 'reverse synchrony' effect in eyewitness identification performance reported in Chapter 2. Rather, I found that evening-type participants performed better compared to morning types, irrespective of time of testing. Evening time-of-day preference has been previously linked to superior performance in holistic information processing. Evening types also appear to be better at processing non-verbal and emotional 
stimuli (Fabbri, Antonietti, Giorgetti, Tonetti, \& Natale, 2007). Both factors are known to be relevant for face processing (e.g., Haxby, Hoffman, \& Gobbini, 2002; Tanaka \& Farah, 1993). It is possible that superior performance of evening compared to morning types resulted from the differences in information processing styles between the two chronotypes.

Several explanations may account for the discrepancy of my findings with previously reported synchrony effect patterns in recognition of non-facial stimuli. First, my results imply that face recognition performance might be immune to 'classical' circadian patterns found in other types of recognition memory. This would certainly not be the first instance of such dissociation: Processing of faces is traditionally considered to be a highly specialized function that is relatively independent from other types of information processing, and examples of selective impairments in one of the memory domains with others remaining intact are well-known (Robotham \& Starrfelt, 2017). This dissociation may also result in different circadian patterns in face recognition performance as opposed to verbal and pictorial recognition. This possibility could be tested in future research using a face recognition paradigm.

Alternatively, the effect size of time-of-day optimality on face recognition performance could in reality be smaller than I anticipated based on prior research that used non-facial stimuli. Hence, the fact that I did not observe the synchrony effect should not necessarily be interpreted as evidence of absence of circadian variations in face recognition performance per se. A face recognition paradigm with multiple recognition trials could offer more statistical power to detect such an effect, if present, compared to the eyewitness memory paradigm with only few identification decisions.

My additional interest was in examining whether testing at the non-optimal time of day magnified unwanted influences of factors that are known to have a biasing effect on eyewitness identifications. To explore this possibility, I exposed some of the participants to a mug shot of an innocent suspect prior to administering the lineup. This manipulation has previously been used to generate biased outcomes in subsequent identification in a scenario when a lineup includes the photograph of the aforementioned innocent suspect (Deffenbacher, Bornstein, \& Penrod, 2006). My interest was in testing whether this biasing effect would be more pronounced in the non-optimal sessions. However, the mug shot manipulation did not appear to be successful. The failure to replicate mug shot exposure effect in this experiment can be linked to a short retention interval between exposure to mug shots and a subsequent lineup identification, which was about 20 minutes in my experiment as opposed to a minimum of 48 hours and up to a week in prior research (e.g., Brown, Deffenbacher, \& Sturgill, 1977; Goodsell, Neuschatz, \& Gronlund, 2009; Memon, Hope, Bartlett, \& Bull, 2002; Perfect \& Harris, 2003). Anticipating the difficulties of conducting multi-session experiments in the MTurk environment, I refrained from using an experimental design with a long retention interval between mug shot exposure and identification decisions. Future research employing a successful biasing manipulation can investigate whether non-optimal testing can magnify the detrimental effect of biasing factors such as mug shot exposure on identification accuracy.

I was also interested in investigating whether time-of-day optimality would affect the postdictive value of confidence and decision times. Based on the optimality hypothesis (Deffenbacher, 
1980), I expected a stronger confidence-accuracy relationship in choosers at the optimal compared to the non-optimal time of day. Results, however, showed an opposite effect: the postdictive value of confidence was stronger in participants that were tested at circadian troughs compared to circadian peaks. These data provide further support for a theory-driven confidence judgments hypothesis as a potential explanation of the way time-of-day optimality affects confidence-accuracy relationship (Palmer, Brewer, Weber, \& Nagesh, 2013). This hypothesis states that, if aware of the factor that negatively affects memory, participants may take this information into account and adjust their metamemory judgements appropriately. This might have been the case in the current experiment; that is, my participants may have been aware of the possible cognitive impairments due to the circadian troughs and taken this information into account when indicating their post-decision confidence.

In Chapter 2, I found that decision times were predictive of accuracy only in optimal sessions. In the current experiment, however, decision times had no postdictive value: I did not observe a commonly found effect where accurate choosers make faster identification decisions than inaccurate choosers (Brewer \& Wells, 2006; Dunning \& Stern, 1994; Sauerland \& Sporer, 2007, 2009). This may indicate that assessing the predictive value of decision times in MTurk studies can be problematic due reduced control over the experimental environment.

It is important to note that my findings are limited to situations when other encoding and retrieval instructions are optimal. Real-life eyewitnesses may often encode events under less favorable conditions, such as insufficient lighting (Wagenaar \& Van Der Schrier, 2008), suboptimal distance (Lindsay, Semmler, Weber, Brewer, \& Lindsay, 2008; Loftus \& Harley, 2005), or short exposure duration (Memon, Hope, \& Bull, 2003). Some of these factors may be more pronounced at circadian arousal troughs, pointing out numerous perspectives for future research. Finally, elderly eyewitnesses are generally poor eyewitnesses (see Fitzgerald \& Price, 2015, for a recent meta-analysis) and are known to have strong time-of-day preferences (Adan et al., 2012; Monk \& Buysse, 2014; Monk et al., 1991). Future studies can test the possibility that obtaining testimony during circadian peaks may partially compensate for this age-related decline in performance in older eyewitnesses.

It is important to identify factors that may result in lower quality of testimony and, whenever possible, ensure most optimal retrieval conditions for eyewitnesses. The severe consequences of memory errors in eyewitnesses make it critical to closely examine factors that can potentially contribute to their memory performance. I tested whether time-of-day optimality could be one of the factors of high relevance to the eyewitness memory field. Based on the combined results of Chapters 2 and 3, I found no evidence supporting the idea that it is possible to increase identification accuracy of eyewitnesses by administering lineups at the peak hour of the day. In other words, with respect to timeof-day optimality, neither the time at which the event was witnessed nor the timing of the lineup administration appears to affect identification outcome in morning- and evening-type eyewitnesses. 
CHAPTER 4

\section{Time-of-day Effects on Face Recognition and Source Monitoring Performance}




\begin{abstract}
The circadian rhythm regulates arousal and activity levels throughout the day and determines hours of optimal cognitive performance. Thus far, circadian fluctuations in face recognition performance received little attention in the psychological literature. The current experiment investigated the effects of time-of-day optimality on the ability to recognize faces and discriminate between the contexts in which where they were encountered. Morning- and evening-type participants $(N=91)$ encoded faces presented in a crime-related and a neutral context either at their optimal or the non-optimal time of day. We expected that testing during circadian peaks as opposed to circadian troughs would result in better recognition performance and ability to exclude familiar but irrelevant faces from the neutral context. Contrary to our hypotheses, results showed no benefit from testing at circadian peaks. I discuss peculiarities of face processing that could account for the discrepancy of my findings with word recall and recognition literature.
\end{abstract}


Have you ever recognized a person but were unable to recall where you knew them from? This common experience reveals that familiarity is not always accompanied by the correct recollection of the memory source (Johnson, Hashtroudi, \& Lindsay, 1993; Yonelinas, 2002). In everyday life the consequences of such source memory errors are mostly harmless. However, in legal contexts the costs can be severe. For example, an innocent suspect may feel familiar to an eyewitness from mug shot viewings, repeated identification procedures, or because they were a bystander at the crime scene (Deffenbacher, Bornstein, \& Penrod, 2006). Attributing familiarity to the incorrect source can lead to eyewitness misidentifications and even wrongful convictions (Lindsay, 2007), calling attention to empirically assess factors that might increase the risk of such memory errors. Time of day could be one such factor: Due to the functioning of our body clock, certain hours of the day are associated with a decline in cognitive performance, making us susceptible to memory errors (Schmidt, Collette, Cajochen, \& Peigneux, 2007). This observation motivated us to investigate whether testing at non-optimal time of day could impact general face recognition performance, as well as the susceptibility to produce errors in source memory for faces.

Our body clock, also known as the circadian rhythm, coordinates numerous aspects of our physiology, cognition, and behaviour (Halberg et al., 2003; Refinetti, 1999). Among other things, the circadian rhythm determines optimal times for engaging in various activities and modulates cognitive performance levels across the day. Generally, we perform better at circadian peaks and worse at circadian troughs (Schmidt et al., 2007). The exact timing of circadian phases can vary across individuals, resulting in inter-individual differences in periods of the day that are optimal (Adan et al., 2012; Horne \& Östberg, 1976). The so-called morning types perform best early in the day, whereas evening types peak in the evening hours (Goldstein et al. 2007; Levandovski, Sasso, \& Hidalgo, 2013). This pattern is known as the synchrony effect: Performance varies as a function of synchrony, or alignment of the time when certain task is performed with the ongoing circadian phase (e.g., May, 1999; May \& Hasher, 1998; Nowack \& Van Der Meer, 2018).

Performance on long-term memory tasks also follows the standard synchrony effect pattern, with better performance at circadian peaks as opposed to circadian troughs. Recall of words and prose passages (Petros, Beckwith, \& Anderson; 1990; Ryan, Hatfield, \& Hofstetter, 2002), sentence and word recognition (May, Hasher, \& Stoltzfus, 1993; Ryan et al., 2002), and cued recall performance in a stem completion tasks (May, Hasher, \& Foong, 2005; Puttaert, Adam, \& Peigneux, 2018) have been shown to be better at the hours of the day that are aligned with participants' chronotype. Such performance fluctuations can be explained by circadian cycles in arousal that are closely linked with amount of attentional resources available to us across the day (Hirst \& Kalmar, 1987; Necka, 1997). Conditions for encoding and retrieval of new memories are most optimal at circadian peaks, when arousal is highest. Circadian arousal troughs, by contrast, may not provide sufficient cognitive capacities necessary to engage in efficient cognitive processing, potentially leading to inferior memory performance (Nowack \& Van Der Meer, 2018). 
Not all manifestations of memory rely on our attentional resources to the same extent. Socalled implicit memory processes that operate outside of our awareness appear to be affected by the circadian rhythm in a different manner than standard recall and recognition tasks. For instance, priming seems to be affected by circadian rhythm differently than cued recall. When explicitly instructed to use previously studied words on a stem completion task, participants usually show a classic synchrony effect pattern. Implicit memory tests, by contrast, generally demonstrate that automatic influences of memory are unaffected by circadian variations in arousal (Puttaert, Adam, \& Peigneux, 2018; Yang, Hasher, \& Wilson, 2007). In fact, one study reported a reversed synchrony effect, with stronger priming at the nonoptimal compared to the optimal time of day (May, Hasher, \& Foong, 2005).

To recap, the circadian rhythm affects our conscious, intentional use of memory, which is often superior at circadian peaks as opposed to circadian troughs. Unconscious influences of memory, however, seem to be unaffected by testing optimality. A common explanation for this dissociation relates to the fact that automatic processes require little to no cognitive resources for their execution, and, therefore, can remain unaffected by many factors that generally impair cognition (Hasher \& Zacks, 1979, 1984). By contrast, controlled, conscious memory processes heavily rely on available cognitive resources for their execution. Performance on tasks that rely on these processes is impaired when cognitive resources are depleted or limited (Smith \& DeCoster, 2000). This also appears to be the case with timeof-day optimality: Reduced cognitive capacity at circadian troughs impairs performance on tasks with a stronger controlled component (e.g., May et al., 1993; Petros et al., 1990; Ryan et al., 2002), whereas automatic memory processes remain intact (May et al., 2005; Yang, Hasher, \& Wilson, 2007).

Providing eyewitness testimonies relies on a mixture of automatic and controlled memory processes. If presented with the culprit during an identification procedure, the eyewitness may be able to engage in an effortful, controlled process known as recollection in order to retrieve some qualitative information about the event (e.g., "I remember this man: He was holding the gun"). If recollection fails, the culprit may still be likely to be selected merely based on familiarity. This is a classic example of an automatic, effortless memory process ("I recognize this person - he must be the man from the crime scene"). In fact, automatic and controlled processes oftentimes work together in these scenarios to produce a correct recognition decision: The feeling of familiarity is complemented by recollection, resulting in an accurate identification. In other words, the 'teamwork' of automatic and controlled processes is beneficial when two memory systems point in the same direction.

For comparison, let us consider a scenario in which an innocent suspect becomes part of a lineup and feels familiar to the eyewitness. The suspect may have been present at the crime scene as a bystander, or their face may have become familiar from previously administered identification procedures or mug shot viewings (Deffenbacher et al., 2006). In these scenarios, a response based solely on familiarity could result in an eyewitness misidentification. An accurate identification decision requires correct recollection of the context in which the suspect was encountered before ("They already showed me a mug shot of this man two weeks ago"). 
Memory for the context in which information was encoded is often referred to as source monitoring (Johnson et al., 1993). Generally, source monitoring tasks are more difficult than standard recognition tasks, and impairing factors (i.e., factors that negatively affect our attentional capacities) can have a disproportionate impact on source monitoring compared to recognition performance (for a review, see Mitchell \& Johnson, 2009). This happens because the two tasks differ in the proportion of automatic and controlled influences of memory on task performance. Source monitoring relies to a large extent on controlled, effortful processes, whereas recognition decisions can be made based solely on a familiarity response that occurs automatically. As impairing factors affect recollection but leave familiarity intact, they produce larger impairments in source monitoring compared to recognition memory (Yonelinas, 1999).

In the current experiment, we investigated whether time-of-day optimality impairs face recognition and source monitoring of faces. We tested morning- and evening-type participants either at their optimal or the non-optimal time of day. In order to model a situation in which a person encountered in a context other than the crime could be misidentified based on the feeling of familiarity, the face stimuli were presented to participants in two distinct contexts: a crime-related context and a neutral context. At recognition, participants indicated whether they had encountered the face earlier in the experiment. Based on prior research showing decline in performance on recognition tasks at circadian troughs as opposed to circadian peaks (e.g. May et al., 1993), we expected diminished recognition performance when participants were tested at their non-optimal versus optimal time of day. Specifically, we expected higher hit (Hypothesis 1a) and lower false alarm rates (Hypothesis 1b) at the optimal compared to the non-optimal time of day. In the source monitoring task, we asked participants to exclusively identify faces from the crime-related context. We hypothesised that participants tested at circadian peaks would outperform those tested at circadian troughs, that is, they would recognize more faces from the crime-related context and would be more successful in excluding faces from the irrelevant neutral context (Hypothesis 2).

\section{Method}

\section{Participants}

To determine the required sample size, we conducted an a-priori power analysis for a one-tailed $t$ test with G*Power v3.1 (Faul, Erdfelder, Buchner, \& Lang, 2009; Faul, Erdfelder, Lang, \& Buchner, 2007). Based on earlier results (May et al., 2005), we expected an effect size of $d=0.52$. We used an alpha error probability of .05 and a power of .80, which resulted in a required sample size of 94.

We pre-screened 346 individuals for their chronotype using the Morningness-Eveningness Questionnaire (MEQ; Horne \& Östberg, 1976). We invited morning- and evening-type individuals for participation in the experiment. Ninety-one participants were tested ( 25 male, 66 female; age 18 to 54, $M=22.13, M d n=21$ years), resulting in a power of .79 to detect an effect of the expected size. The sample included university students $(n=87)$ and members of the general public $(n=4)$. Among them, $57.1 \%$ were evening types $\left(n=52, \mathrm{MMEQ}_{\mathrm{ME}}=34.37, S D_{\mathrm{MEQ}}=5.05\right)$ and $42.9 \%$ were morning types $(n=39$, 
$M_{\text {MEQ }}=63.77, S D_{\text {MEQ }}=4.46$ ). We only included Caucasian participants to avoid cross-racial bias (Wilson, Bernstein, \& Hugenberg, 2016).

\section{Materials}

Pre-screening questionnaire. We used the full version of MEQ (Horne \& Östberg, 1976) to determine participants' chronotype. The MEQ consists of 19 questions about participants' sleeping habits, alertness levels at different points of the day, and preferred times for engaging in physically and cognitively demanding tasks (e.g., "At what time in the evening do you feel tired and in need of sleep?"). The MEQ score correlates with daily changes in melatonin and cortisol levels, body temperature (Baehr, Revelle, \& Eastman, 2000; Horne \& Ostberg, 1976), sleep habits and activity levels (Andrade, BeneditoSilva, \& Menna-Barreto, 1992; Bailey \& Heitkemper, 2001; Duffy, Rimmer, \& Czeisler, 2001). The MEQ items in the pre-screening were intermixed with filler questions about food and caffeine consumption habits and sleep to provide additional support for the cover story.

Face stimuli. I used face stimuli from the Karolinska Directed Emotional Faces database (Lundqvist, Flykt, \& Öhman, 1998) for the current experiment. The database contains photographs of 70 male and female amateur actors displaying different emotional expressions (in the current experiment, we only used neutral-expression versions). All the actors were between 20 and 30 years old and were wearing the same grey T-shirt. When the stimuli were prepared, the actors were not permitted to wear earrings or eyeglasses, visible makeup, beards or moustaches. Importantly, for each of the actors, the database includes two versions of each facial expression taken at two separate occasions. Having two different photographs of the same person available allowed me to use different photographs at encoding and at test, which is a preferable way of testing face recognition performance (Burton, 2013).

For my experiment, I selected photographs of 64 faces with a neutral emotional expression. A total of 32 faces (16 male, 16 female) were used as targets. They were further divided into two sets 16 faces each ( 8 male, 8 female) that were rotated through the context (crime-related vs neutral). Faces were presented against a background image depicting the respective context (see Figure 1). For each context, I created four versions of each face set with the order of faces randomized to control for order effects in memory performance. In each encoding context, participants were presented with one of the four versions that was selected randomly.

For the memory tests, each subset was further divided into two subsets consisting of eight faces ( 4 male, 4 female) that were rotated through the test conditions (recognition task vs source memory task). I also selected 32 faces that were used as filler faces in the memory tests. They were divided into two sets 16 faces each ( 8 male, 8 female) and were rotated through the two tasks (recognition task vs source memory task). The full counterbalancing table can be found in Appendix B.

\section{Design}

Optimality (optimal vs non-optimal) served as a between-participants factor and encoding context (crime-related vs neutral) served as a within-participants factor. We manipulated encoding context by embedding face stimuli into crime-related and neutral scenarios. In the recognition task, 
participants indicated whether they had seen each face earlier in the experiment, irrespective of the context. In the source monitoring task, participants had to exclude faces from the neutral context and indicate recognition for faces from the crime-related context. Proportions of yes responses to studied (hits) and unstudied faces (false alarms) from each context served as the outcome variables.

We used hit and false alarm rates to calculate discrimination accuracy and response bias. Discrimination accuracy $\left(d^{\prime}\right)$ indicates participants' ability to distinguish signals (studied faces) from noise (fillers). A value of zero indicates zero ability to distinguish studied faces from fillers, whereas a value of 4.65 is considered an effective ceiling (Macmillan \& Creelman, 2009). Response bias (c), on the other hand, reflects participants' threshold for deciding that they have seen the face before. A positive $c$ value shows a bias towards saying no, whereas a negative $c$ value reflects participants' tendency to indicate that they have seen the face before (Green, 1966).

\section{Procedure}

Participants were recruited via advertisements at university campus. The cover story held that the experiment concerned differences in food and caffeine consumption habits between morning- and evening-type individuals and their effect on memory performance. Morning sessions took place between 7:40 AM and 9:00 AM, and evening sessions were scheduled between 8:30 PM and 9:30 PM.

We instructed participants not to consume alcohol 18 hours prior to testing, avoid caffeine and chocolate on the day of testing and schedule participation only if they slept a minimum of six hours in the night prior to testing. Participants confirmed compliance to these criteria and signed the consent

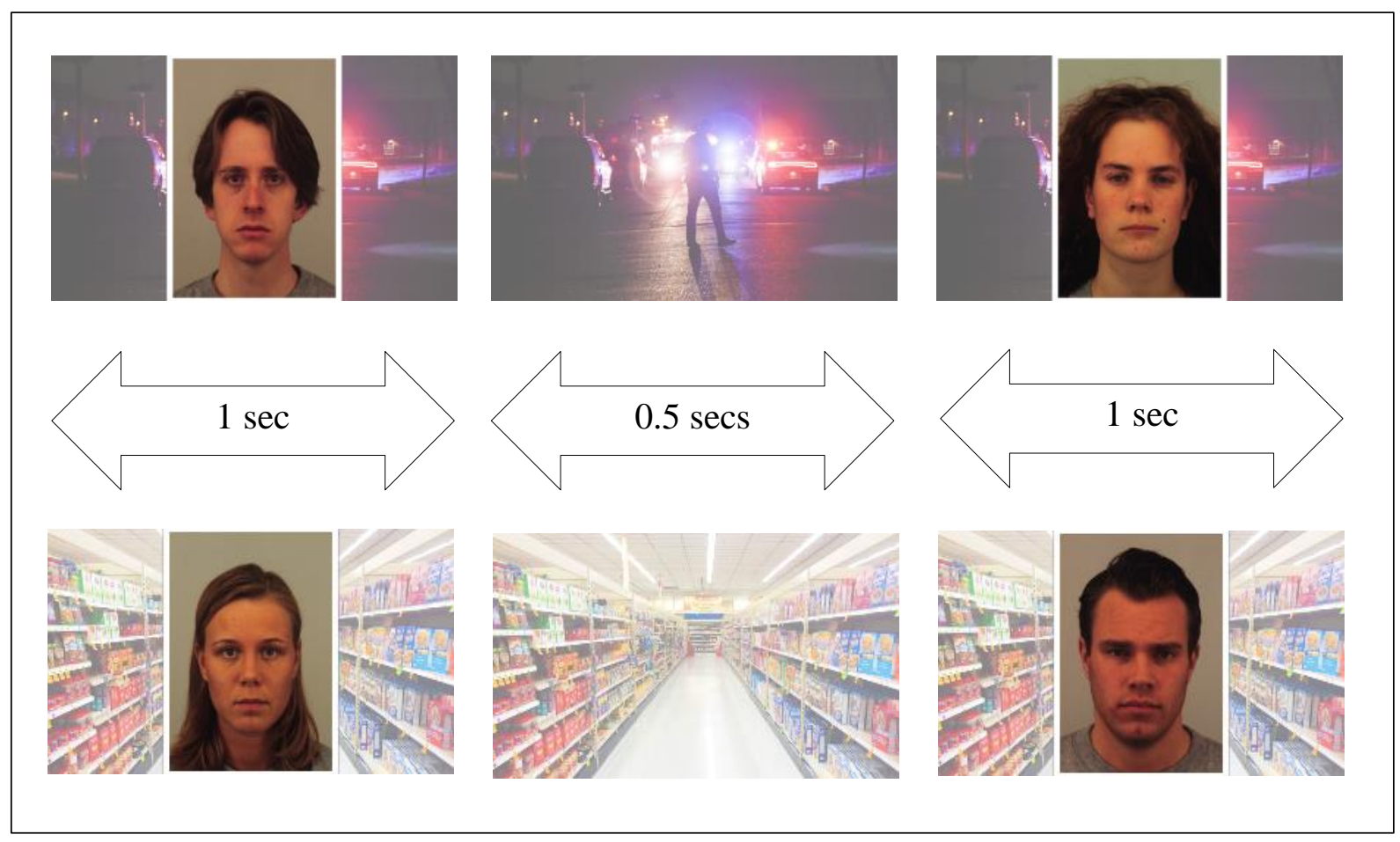

Figure 1. Example of encoding stimuli presented in crime-related context (top row) and neutral context (bottom row). 
form at the beginning of the testing session. Next, we presented participants with the crime-related scenario:

Imagine waiting for a bus late at night. There has been an important football derby in your town and you notice a large group of football hooligans that are behaving violently. They approach a single fan of the other team and start insulting him. Very quickly the encounter becomes violent: the hooligans start pushing and beating him. You decide to call the police. You will now be presented with the faces of the hooligans. Pay attention - you may be asked to identify them later.

These instructions were accompanied by a context-cue: a photograph of a dark street with police cars and a policeman. Figure 1 depicts an example. After that, I informed participants that they would be presented with the faces of the hooligans and instructed them to pay attention as they may be asked to identify them later. Participants saw 16 faces presented against the background that accompanied the imagery instructions, one at a time for $1 \mathrm{~s}$ followed by a $0.5 \mathrm{~s}$ interval.

Following a 5-min filler task (object search), we introduced the neutral context:

Imagine heading out to the supermarket on a rainy day. You drop off your beloved umbrella that you received as a gift from your grandmother at the entrance. After you finished shopping, you want to pick up your umbrella - but you realize that it's gone! However, you notice another umbrella that is very similar to yours. Someone must have mistaken their umbrella with yours and taken it by accident. You will now see the people you've encountered in the supermarket while shopping. Pay attention - one of them might have your umbrella!

These instructions were accompanied by an image of a supermarket aisle. We told participants that they would now see the people they encountered in the supermarket and again told instructed them to pay attention to the faces as one of them might have their umbrella. The faces were presented against the background of the supermarket aisle.

Following another 5-min filler task (object search) and questionnaire about their sleep, food and caffeine consumption habits, participants received the recognition and the source monitoring tasks. In the recognition task, I reminded participants of the pictures they encoded earlier and asked them to indicate whether they had seen each of the faces in either context. Participants indicated if they had or had not seen each face earlier in the experiment. In the source monitoring task, I told participants that, unlike in the previous task, they should only select the faces previously seen in the crime-related context (i.e., the hooligans). Participants had unlimited time for their decisions. Participants received 5-euro gift vouchers or participation credit for their participation. We debriefed participants upon the completion of the data collection.

\section{Results}

\section{Recognition Memory}

I conducted independent-samples Welch t tests to determine if there were differences in overall hit rates (hypothesis 1a) and false alarm rates (hypothesis $1 \mathrm{~b}$ ) between optimal and non-optimal time of day on the recognition task. Additionally, I computed JZS Bayes factors as a measure of a degree 
to which our data favors the null over the alternative hypothesis (Jeffreys, 1961). I applied default Cauchy's prior with scaling factor of 0.707 . There was no statistically significant difference in hit rates at optimal $(M=.47, S D=0.20)$ versus non-optimal $(M=.51, S D=0.20)$ time of day, $t(86.01)=-0.924, p=$ .821, $d=-0.19, B F_{01}=8.03$. Likewise, false alarm rates did not significantly differ between optimal $(M=$ $.22, S D=0.16)$ and non-optimal sessions $(M=.25, S D=0.15), t(87.88)=-1.173, p=.878, d=-0.25, B F_{01}=$ 9.04. The Bayes factors suggest moderate evidence in support of the null hypothesis (Lee \& Wagenmakers, 2013). Signal Detection Theory analyses showed that sensitivity (i.e., participants' ability to differentiate targets and non-targets) was $d^{\prime}=0.81$ at the optimal time of day and $d^{\prime}=0.76$ at the non-optimal time of day. The referring response bias (i.e., the thresholds for deciding that the target was encountered before) was $c=0.95$ and $c=0.74$, respectively. These findings lend no support to hypotheses $1 \mathrm{a}$ and $1 \mathrm{~b}$.

\section{Source Monitoring}

Table 7 presents mean proportion of yes responses to faces from each context across the experimental conditions. We ran a two-way mixed ANOVA with optimality (optimal vs non-optimal) and context (crime-related vs neutral) as factors to test whether optimality affected participants' ability to exclude irrelevant faces on the face recognition task (hypothesis 2). Proportions of 'yes' responses to studied faces served as the dependent variable. The two-way context $x$ optimality interaction was not significant, $F(4,89)=0.430, p=.514$, partial $\eta^{2}=.005$. The main effect of context was significant, $F(2,89)$ $=14.353, p<.001$, partial $\eta^{2}=.139$ : Participants provided more yes responses to faces from crimerelated than neutral context $\left(M_{\text {crime }}=.48\right.$ and $\left.M_{\text {neutral }}=.41\right)$. The main effect of optimality was not significant, $F(2,89)=0.857, p=.357$, partial $\eta^{2}=.010$. These data do not support Hypothesis 2 . Figure 2 shows participants' performance on face recognition and source monitoring task at the optimal and the non-optimal time of day.

Table 7

Mean proportion of Yes responses [and 95\% CI] to studied faces in crime-related and neutral contexts and to non-studied faces in the face recognition and source monitoring task as a function of time of testing.

\begin{tabular}{llcc}
\hline & Context & \multicolumn{2}{c}{ Time of testing } \\
& Crime-related & Optimal & Non-optimal \\
\hline \multirow{2}{*}{ Recognition task } & Neutral & $.52[.46 ; .58]$ & $.55[.48 ; .61]$ \\
& Non-studied & $.42[.35 ; .49]$ & $.47[.40 ; .55]$ \\
& Crime-related & $.22[.17 ; .26]$ & $.25[.21 ; .30]$ \\
Source monitoring task & Neutral & $.44[.37 ; .51]$ & $.42[.34 ; .49]$ \\
& Non-studied & $.34[.28 ; .40]$ & $.40[.33 ; .46]$ \\
& & $.17[.13 ; .21]$ & $.19[.14 ; .25]$ \\
\hline
\end{tabular}




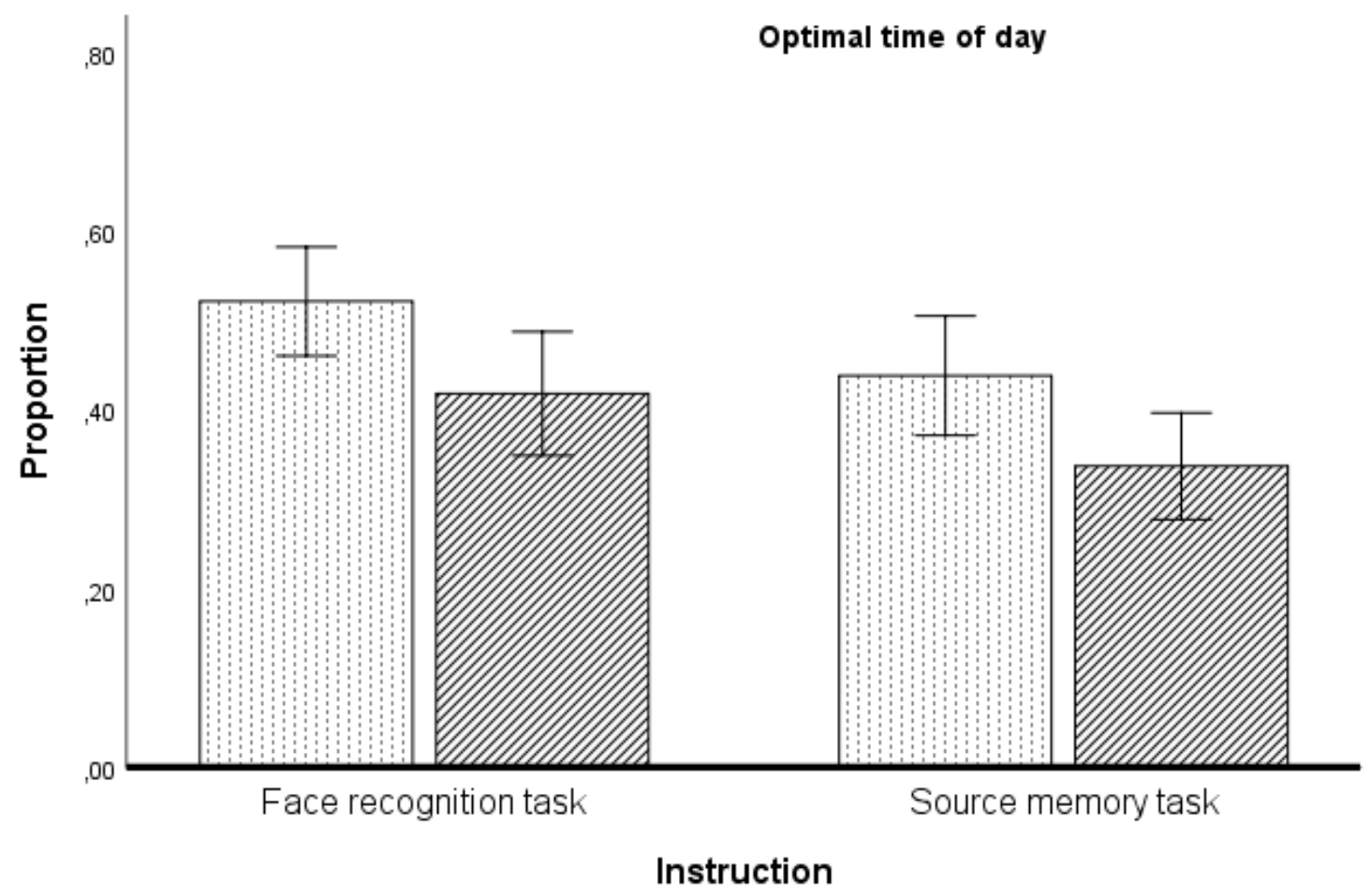

\section{Context}

Griminal

Ⓝeutral

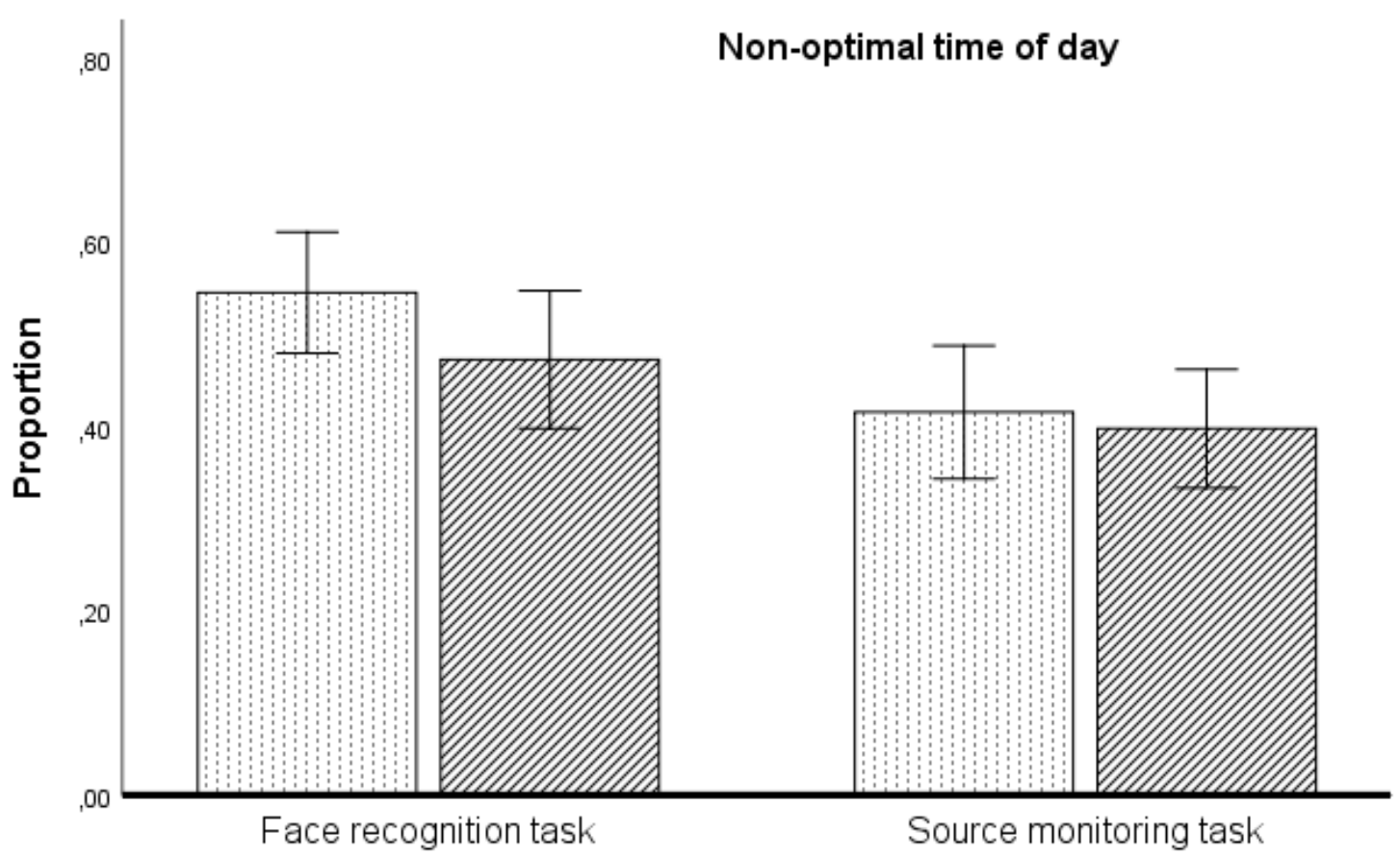

Figure 2. Proportion of Yes responses to faces encoded in each of the two contexts in the recognition and source memory task (and 95\% $\mathrm{Cl}$ error bars) at optimal and non-optimal time of day. 


\section{Discussion}

I tested whether face recognition and source monitoring performance varied as a function of time of day. Previous studies showed superior recall and recognition performance at those hours of the day that were aligned with individual's peak arousal hours, as determined by the circadian clock (e.g., Anderson et al., 1991; May et al., 1993; Petros et al., 1990). The current experiment extended earlier work by investigating the synchrony effect specifically in face recognition and source memory performance for faces. I hypothesised that face recognition performance would be superior at circadian peaks as opposed to circadian troughs (Hypothesis 1). I also expected to find the synchrony effect in source monitoring performance (Hypothesis 2). Surprisingly, our results showed no evidence of such synchrony effect in either test. Participants performed quite similarly across optimal and non-optimal testing sessions. These observations contrast with findings reported in previous studies that used nonfacial stimuli to examine the synchrony effect in episodic memory performance (Schmidt et al., 2007).

What can be the explanation for the observed findings? From a cognitive perspective, processing of faces differs from processing of other types of information (Bruce \& Young, 2012; Robotham \& Starrfelt, 2017; Schwartz, 2014). Faces are processed holistically (e.g., Richler \& Gauthier, 2014) and are distinct in terms of allocation of attention during encoding. For instance, when presented with two faces simultaneously, encoding of one of the faces requires us to suppress processing of features of the other face, whereas this is not the case when we are simultaneously presented with two objects (e.g., Bindemann, Jenkins, \& Burton 2007; Boutet \& Chaudhuri, 2001; Palermo \& Rhodes, 2002). Considering that circadian variations in cognitive performance are generally construed in terms of availability of attentional capacities (Valdez, 2019) and abilities of efficient allocation of cognitive resources (e.g., Nowack \& Van Der Meer, 2018), the specifics of distribution of attention in encoding of faces may account for the fact that the circadian performance cycles are expressed differently in face recognition performance.

From a neuroscientific perspective, face recognition relies on specialized areas of the brain different from areas involved in other types of recognition memory (Schwartz, 2014). Areas of our brain responsible for memory functioning show oscillations that are autonomous from those generated in the suprachiasmatic nuclei, also known as the central pacemaker of our body (Snider, Sullivan, \& Obrietan, 2018). However, little is known about autonomous circadian cycles in the specialized areas responsible for face processing. The divergent pattern of daily fluctuations in face recognition compared to recognition of other types of stimuli could be linked to the fact that peripheral oscillations in the brain areas responsible for face processing function in a different manner. The combination of brain imaging techniques with the forced desynchrony protocol, which involves isolation from external time cues and imposition of a 'day' with a duration other than 24 hours (e.g., 28 hours; Wright, Hull, Hughes, Ronda, \& Czeisler, 2006), may offer promising discoveries about these dissociations.

One limitation of this study concerns overall low performance in the memory tests. Hit rates were low, though performance in target-absent trials was better, resulting in overall low sensitivity in both optimality conditions with small negative bias. It seems that participants found the task overall 
difficult, which may have masked possible effects of time-of-day optimality. Indeed, numerically, participants provided lower rates of yes responses for faces from neutral compared to crime-related context at the optimal but not at the non-optimal time of day; however, the difference was not statistically significant. Future studies might address this issue by increasing exposure duration, which should result in stronger encoding and better overall performance. Another limitation concerns the fact that faces from the crime-related context produced better performance than faces from the neutral context. Crime-related faces were presented first, possibly causing this difference in performance across the two contexts. Alternatively, participants may have perceived crime-related faces as more important. Even though the differences in memory strength for faces from the two contexts do not compromise our results, counterbalancing the order of contexts in future studies would allow to avoid differences in performance rates resulting from order effects.

To conclude, this work provides a first investigation of the possible circadian effects on face recognition performance. The current findings cautiously suggest that face recognition performance may not follow the standard synchrony effect patterns observed in memory for non-facial stimuli. It remains unclear whether this immunity to daily fluctuations in performance is a result of cognitive and neural mechanisms underlying face processing, which outlines important directions for future research. Aditional research is also necessary to test the possibility of the synchrony effect in older populations, which are known to have age-related decline in memory performance (Fitzgerald \& Price, 2015) and are more prone to source monitoring errors (e.g., Benjamin \& Craik, 2001; Schacter, Kaszniak, Kihlstrom, \& Valdiserri, 1991; Trott, Friedman, Ritter, Fabiani, \& Snodgrass, 1999). 
CHAPTER 5

General Discussion 
Chapter 5 
Accurate and informative evidence provided by eyewitnesses can play a pivotal role in investigations in many criminal cases (Peterson, Sommers, Baskin, \& Johnson, 2010; Ridley, Gabbert, \& La Rooy, 2013; St-Yves, 2015). However, eyewitness testimony can be unreliable, resulting in counterproductive investigative actions, miscarriages of justice, and dramatic failures to meet the global objectives of the law (Clark, Benjamin, Wixted, Mickes, \& Gronlund, 2015; Forst, 2013; Wagenaar, 2009). The incidence of prosecution of innocent individuals resulting from erroneous eyewitness identification is alarmingly high. For instance, mistaken eyewitness identifications alone are involved in over two thirds of known cases of wrongful convictions in the United States (Innocence Project, 2020). These facts necessitate a close examination of factors that can potentially impact memory performance in eyewitnesses.

Basic laboratory research often marks directions for the investigation of such factors by identifying general principles of cognition. The applied methodologies used in the eyewitness memory literature, on the other hand, make it possible to study how the general principles of memory functioning manifest themselves in (almost) real-life eyewitnesses. As an example, consider the reconstruction principle of memory (Bartlett, 1932). The discovery of this principle offered theoretical impetus for uncovering the dangers of exposing eyewitnesses to post-event misinformation (Loftus, 2005; Loftus, Miller, \& Burns, 1978) and contributed to the development of evidence-based recommendations for investigative interviewing protocols (e.g., Gabbert, Hope, \& Confrey, 2018). The eyewitness memory field is rich in examples of basic laboratory discoveries being used to strengthen our understanding of the eyewitness memory phenomena (Wells, 1978).

Despite the significant strides that have been made in the fundamental and applied memory literature (e.g., Wells, Memon, \& Penrod, 2006; Wells \& Olson, 2003), the relevance of other potential contributors to eyewitness memory performance remains unknown. The so-called synchrony effect in cognitive performance is one of such unexplored potential contributors. It is now a general agreement in the psychological literature that the amount of cognitive capacities available to us is not constant across the day (Schmidt, Collette, Cajochen, \& Peigneux, 2007). Driven by our body clock, our performance at certain hours of the day is better compared to others (Valdez, 2019). Yet, criminal activity takes place around the clock (e.g., Felson \& Poulsen, 2003), and one may become a witness to a crime at any moment of the day. It is therefore inevitable that some eyewitnesses rely on their memory for events they saw during hours when their capacity to encode new information was perhaps poor. Furthermore, obtaining testimony during suboptimal performance periods could lead to further impairments in accuracy and informativeness of eyewitness statements and identifications.

In basic laboratory experiments, recall and recognition performance is consistently better at the optimal compared to the non-optimal hours (e.g., May, Hasher, \& Foong, 2005; Petros, Beckwith, \& Anderson; 1990; Puttaert, Adam, \& Peigneux, 2018; Yang, Hasher, \& Wilson, 2007). Table 8 presents an overview of the sizes of the time-of-day optimality effects observed in the previous literature. The effect sizes range from small to medium, with an average effect size $d=1.02$. The average size of time-of-day optimality effect in the reviewed studies is comparable, for instance, to the sizes of effects of high 
relevance to the eyewitness memory field, such as biased identification instructions or weapon focus effect (Steblay, 1992, 1997). Therefore, previous literature on the synchrony effect in memory performance suggests potential relevance of time-of-day optimality to the eyewitness memory field. Nonetheless, this hypothesis had not previously been tested in a systematic matter using the eyewitness memory paradigm. The current research programme presents the first comprehensive attempt to empirically test possible time-of-day optimality effects across various aspects of eyewitness memory performance.

Table 8

Effect sizes observed in published experiments that examined the synchrony effect in memory performance

\begin{tabular}{lllcl}
\hline \multicolumn{1}{c}{ Authors } & $\begin{array}{l}\text { Sample } \\
\text { size }\end{array}$ & Task / stimulus type & p-value & $\begin{array}{l}\text { Effect size } \\
\text { (Cohen's } d \text { ) }\end{array}$ \\
\hline $\begin{array}{l}\text { May, Hasher, \& Foong } \\
\text { (2005) }\end{array}$ & 82 & Stem completion & 0.18 & 0.52 \\
$\begin{array}{l}\text { May, Hasher, \& } \\
\text { Stoltzfus (1993) }\end{array}$ & 34 & Sentence recognition & $<.005$ & 1.23 \\
$\begin{array}{l}\text { Puttaert, Adam, \& } \\
\text { Peigneux (2018) }\end{array}$ & 40 & Estimates of controlled & $<.001$ & 0.95 \\
& & processes in stem & \\
Ryan, Hatfield, \& & 40 & completion & & \\
Hofstetter (2002) & & Free recall (words) & & \\
\hline
\end{tabular}

\section{Summary of the Findings}

In Experiment 1, I compared performance in three different domains of eyewitness memory at the optimal and the non-optimal times of day. After encoding the stimulus events, participants provided free narratives and answered cued questions about the incident and the people involved. Results showed no effect of testing optimality on accuracy of free reports or answers to cued questions. I also asked participants to identify people involved in the incidents. To my surprise, and contrary to previous synchrony effect findings in recognition memory (e.g., May et al., 1993), identification accuracy in targetpresent lineups was unexpectedly higher at the non-optimal time of day. Performance in target-absent lineups was not affected by testing optimality. Decision times were also predictive of accuracy at the non-optimal compared to the optimal time of day, in line with previous data on the effect of circadian arousal on long-term memory access speed (Anderson, Petros, Beckwith, Mitchell, \& Fritz, 1991). The postdictive value of confidence, however, showed some evidence of 'reversed' synchrony: Highly confident choosers were significantly better calibrated at the non-optimal compared to the optimal times of day. Finally, I compared false memory rates in the visual version of the DRM paradigm at the optimal and the non-optimal times of day. Results showed comparable false memory rates across the 
optimality conditions. This finding was in line with previously reported results on the production of false memories in young adults (Intons-Peterson, Rocchi, West, McLellan, \& Hackney, 1999).

The superior identification performance at the non-optimal time of day in Experiment 1 was surprising: Prior research predicted the opposite effect, that is, better performance at circadian peaks. The increase in hit rates was not accompanied by an increase in false-alarm rates, which is a typical pattern in recognition studies (Wixted \& Mickes, 2014). Additionally, the effect was specific to targetpresent lineups and the size of the observed effect was small. These factors guided my interest in testing whether the obtained effect would replicate.

For this purpose, I conducted Experiment 2. Enhancements in the experimental design allowed me to eliminate possible expectancy effects, potential effects of lineup composition, and increase generalizability of my findings to the broader population. In addition, in Experiment $2 \mathrm{I}$ aimed to test the idea that non-optimal testing could magnify proneness to memory biases. For this purpose, I introduced bias into participants' identification decisions by exposing them to a mug shot of an innocent suspect prior to lineup identification. The photograph of the innocent suspect also appeared in the lineups, and I expected participants to erroneously identify him based on familiarity gained during the mug shot exposure (Deffenbacher, Bornstein, \& Penrod, 2006). I expected this negative tendency to be magnified by non-optimal testing. However, mug shot exposure did not produce a biasing effect, making it impossible to assess whether testing optimality had a moderating potential for this negative, and potentially dangerous, effect commonly reported in the literature ${ }^{4}$. Regarding identification performance, I found no significant differences between optimal and non-optimal sessions. Inferential analyses showed that confidence was predictive of accuracy at the non-optimal but not the optimal time of day, paralleling evidence from calibration analysis in Experiment 1.

Strikingly, previously reported patterns of superior performance at the optimal compared to the non-optimal time of day were not replicated in either of the two experiments. What are the possible reasons for these discrepant results considering the seemingly 'classic' synchrony effect patterns reported in the literature? One possibility is that the circadian arousal affects face recognition performance in a different manner than recognition of other types of stimuli. Differential impairments in face processing as opposed to processing of other types of stimuli are well-acknowledged (Bruce \& Young, 2012; Robotham \& Starrfelt, 2017), and my results might suggest that the circadian rhythm is yet another factor that affects face processing in an atypical manner.

In order to further explore this possibility, I used a modification of the face recognition paradigm in Experiment 3. Participants encoded sets of faces and were presented with a recognition test at either optimal or the non-optimal time of day. I expected recognition performance to be better at circadian peaks as opposed to circadian troughs. Multiple recognition trials allowed us to collect a significantly larger number of data points per participant compared to Experiments 1 and 2, offering statistical power

\footnotetext{
${ }^{4}$ I addressed this issue by including a more effective biasing manipulation in Experiment 3 , as discussed below.
} 
to detect even small-size effects. Contrary to my hypothesis, results again showed no evidence of superior recognition performance at the optimal compared to the non-optimal time of day.

The second aim of Experiment 3 was to test whether memory for contexts in which faces were encountered is better at circadian arousal peaks compared to arousal troughs. The use of the face recognition paradigm allowed us to design a more potent biasing manipulation compared to mug shot exposure as used in Experiment 2. Specifically, I embedded face stimuli into two distinct contextual scenarios. Some faces were presented within a context of a criminal scenario, whereas other faces were presented within a neutral context. I then administered a memory test that required participants to discriminate between the two different contexts in which the faces were encoded. Specifically, I asked participants to recognize faces they had seen in the crime-related context, ignoring the faces from the neutral context. I expected that familiarity gained during encoding of neutral-context faces would misguide participants to incorrectly recognize some of neutral-context faces as 'criminals'. My main interest was in testing whether these errors would be more likely to occur in the non-optimal compared to the optimal sessions. Contrary to my hypothesis (but consistent with overall pattern of findings my line of research), participants showed no evidence of significant benefit in source monitoring task from optimal testing.

To summarize, across a battery of memory tests included in the three experiments within the current research programme, I consistently found no evidence of superior performance at circadian peaks as opposed to circadian troughs. Table 9 presents the overview of sizes of time-of-day optimality effects observed across the experiments. All of the effect sizes can be considered small and, if anything, the results point in the opposite direction than predicted. This pattern of findings is contrary to my expectations regarding the effect of time-of-day optimality on memory performance in eyewitnesses.

Table 9

Overview of effect sizes of time-of-day optimality effects across Experiments 1-3

\begin{tabular}{lllrc}
\hline Experiment & $\begin{array}{l}\text { Sample } \\
\text { size }\end{array}$ & Task type & $\begin{array}{c}\text { p- } \\
\text { value }\end{array}$ & $\begin{array}{c}\text { Effect size } \\
\text { (Cohen's d) }\end{array}$ \\
\hline Experiment 1 & 103 & Identification from lineups & .015 & -0.26 \\
Experiment 2 & 315 & Identification from lineups & .202 & 0.09 \\
Experiment 3 & 92 & Face recognition: hit rates & .821 & -0.19 \\
Experiment 3 & 92 & Face recognition: false alarm & .878 & -0.25 \\
& & rates & \\
\hline
\end{tabular}

\section{Interpreting Results}

Task Difficulty. Factors that affect cognitive performance can produce differential levels of impairment across different levels of task difficulty. Tasks that require high cognitive resources for their execution are generally affected by impairing factors to a large extent, whereas tasks that require less effort may be affected less or not affected at all (e.g., Yonelinas \& Jacoby, 2012). The administered 
memory tests in my experiments were not homogeneous in terms of their difficulty, and I expected that non-optimal testing would result in stronger impairments in more difficult tasks compared to easier tests.

The face recognition task in Experiment 3, as well as identification tasks in Experiments 1 and 2 belong to the category of recognition tests. These are generally believed to have lower levels of difficulty compared to other types of memory tests such as recall tests. Recognition tasks provide strong retrieval cues in the form of a representation of the to-be-recognized item. Hence, an accurate response in a recognition test can be based solely upon the feeling that the item seems familiar. Familiarity is an automatic process that requires little to no cognitive resources, which can make it immune to many factors that negatively affect our cognition (Jacoby, 1991). Troughs in circadian arousal are no exception: They appear to have no impairing effect on automatic components of memory functioning (May et al., 2005; Yang et al., 2007). Therefore, the absence of the synchrony effect in recognition tests in my experiments, though contrasting previous findings by May et al. (1993), is less surprising and has parallels in other known memory-impairing effects in cognitive psychology 5 .

The source memory task employed in Experiment 3 had a higher level of difficulty compared to a standard recognition test (Mitchell \& Johnson, 2009). In this task, participants had to discriminate between faces encountered in two different contexts. In order to complete the task, it was necessary to go beyond familiarity-based responses and retrieve qualitative information about the context in which each face was encountered. This process is known as recollection and it differs from familiarity in many important aspects (e.g., Jacoby, 1991). Unlike familiarity, recollection is reliant on available cognitive resources and therefore can be strongly affected by factors that reduce our attentional capacities (Yonelinas, 1999). Recent data shows that the dips in cognitive performance caused by the circadian rhythm act in a similar manner: They appear to affect recollection while leaving automatic memory processes intact (Puttaert et al., 2018).

These findings offer interesting predictions regarding performance on tasks in which familiarity and recollection oppose each other. When a familiarity-based response is incorrect and needs to be ignored based on information retrieved in recollection, suboptimal processing conditions increase the chance of memory errors (for a review, see Yonelinas, 2001). The source memory task in Experiment 3 was specifically designed to create such an opposition between recollection and familiarity. All the studied faces could appear familiar to participants, but some of them belonged to irrelevant context and thus needed to be rejected. I expected that participants would be better at ignoring familiar but irrelevant faces at the optimal time of day, when their cognitive capacities are better. To my surprise, participants in this task showed only minor benefit from optimal testing that was not statistically significant. This finding is not in line with predictions of the synchrony effect model.

\footnotetext{
${ }^{5}$ Consider, for instance, such a robust and reliable factor as age-related decline in memory (Fitzgerald \& Price, 2015). Whereas it is strongly pronounced in recall tasks, it can show no impairments on recognition (e.g., Schonfield \& Robertson, 1966).
} 
Finally, free and cued recall are the most difficult memory tasks I used in my experiments. They provide the lowest amount of retrieval cues and therefore primarily rely upon recollection. Time-of-day optimality has been consistently shown to affect performance on these tasks (e.g., Intons-Peterson et al., 1999; Petros et al., 1990; Puttaert et al., 2018). These patterns are in line with the cognitive capacity explanation of the synchrony effects in cognitive performance: Recollection is an effortful process largely reliant upon cognitive capacities for successful execution (e.g., Schoenfield \& Stones, 1979) and therefore should show most robust impairments during circadian troughs. Nonetheless, free narratives and answers to cued questions in Experiment 1 were not subject to this standard synchrony effect pattern. This may be the most unexpected of my findings: I expected that the synchrony effect in eyewitness memory, if present, should be easily detectable in tests that heavily rely on availability of cognitive capacities.

To summarize, the fact that I did not observe impairing effects of non-optimal testing across a wide battery of memory tests of varying difficulty levels was unexpected from the perspective of the dual process theory of memory performance (Yonelinas, 1999) and contrasts previously reported synchrony effect patterns in laboratory tasks of analogous levels of task difficulty (Schmidt et al., 2007). To interpret these discrepancies, I turn focus to methodological differences between prior research and my experimental protocols.

Encoding Instructions. Similar to the protocol used in previous synchrony effect research (e.g., May et al., 1993, 2005; Petros et al., 1990), participants in my experiments were instructed to play close attention to the presented stimuli and warned about the forthcoming memory test. Nonetheless, certain aspects of the eyewitness memory paradigm may have put the participants in a more beneficial position compared with participants in previously conducted synchrony effect studies.

In Experiments 1 and 2, I informed participants before presenting the stimulus event that they would be asked to serve as eyewitnesses. Compared with a generic warning that participants' memory for the presented stimuli will be tested, this information gives participants more detailed understanding of the nature of the subsequent memory test. Based on common knowledge about eyewitness testimony, the mock witnesses could anticipate that they would be expected to report specific details from the to-be-presented stimuli, such as the appearance of the perpetrator, modus operandi, the sequence of events, etc. Therefore, it is possible that prior knowledge on the nature of the memory test may have affected participants' encoding strategies, encouraging efficient distribution of attentional resources to enhance task-specific retrieval accuracy.

An additional benefit of informing participants about the fact that they would act as eyewitnesses concerns the activation of participants' schemata (i.e., general knowledge, beliefs expectations) of crime. Processing the to-be-encoded stimuli in relation to pre-existing schemata generally has beneficial effects on encoding, including higher accuracy rates (e.g., Hastie \& Kumar, 1979; for a review, see van Kesteren et al., 2012). Previous experiments showing the synchrony effect in memory performance did not involve activation of such shematas that could strengthen memory encoding (e.g., May et al., 1993; Petros et al., 1990; Puttaert et al., 2018). Combined, these factors may 
have resulted in stronger encoding of the presented stimuli compared to previously conducted research showing synchrony effects in memory performance.

Retrieval Instructions. The retrieval instructions in the eyewitness memory paradigm also differ from instructions normally used in previous synchrony effect studies. The idea that one should aim to perform well on a memory test is certainly inherent in any explicit memory task. However, I specifically emphasized the importance of providing as complete and accurate answers as possible. I also explicitly discouraged participants from guessing details they could not remember. Consistent with standard methodologies in eyewitness memory research, identification instructions clearly specified that the perpetrator may or may not be present in the lineup, and I encouraged participants to select the "Not present" option if they were not sure or did not know. Such instructions are known to induce a more conservative response strategy and encourage a neutral position towards the presence of the target in the lineup (Steblay, 1997). No equivalent of such instructions was present in the protocols of previous studies that showed synchrony effects in memory performance (Schmidt et al., 2007).

Stimuli Type. Previous research showing synchrony effect patterns in performance tested memory for encoded prose passages, sentences, word lists, word stems and pictorial stimuli (e.g., IntonsPeterson et al., 1999; May et al., 1993; Puttaert et al., 2018). By contrast, participants in Experiments 1 and 2 encoded stimulus events depicting staged crimes and subsequently identified individuals involved in the incidents. In Experiment 3, I used the face recognition paradigm. The fact that I did not observe a standard synchrony effect pattern in tasks involving recognition of faces may suggest that the circadian rhythm affects processing of faces differently than memory for other types of stimuli.

Face processing is a highly specialized function that differs from other types of recognition (Haxby et al., 2001; Kanwisher, McDermott, \& Chun, 1997). Impairments in the face recognition domain are known to be dissociable from impairments in recognition of non-facial stimuli (Bruce \& Young, 2012; Robotham \& Starrfelt, 2017). My findings may indicate that circadian-related impariments could be another example of such dissociations.

To summarize, the analysis of methodological differences between previously conducted synchrony effect research and my studies reveals important differences in task type, stimulus type, and encoding strategies that might account for the discrepancy between my findings and previously reported data. Notably, all of the discussed factors are specific to the eyewitness memory paradigms and reflect the differences between conditions to which eyewitnesses are exposed in real-life situations as opposed to participants in a standard laboratory experiment. My results highlight theoretical gaps in understanding the mechanisms behind time-of-day fluctuations in memory performance and raise the issue of constraints of generality of standard laboratory research to applied settings, such as eyewitness memory contexts.

\section{Theoretical Contributions}

The synchrony effect and compensatory resource allocation mechanisms. Troughs in cognitive performance are often explained in terms of decreased availability of cognitive resources at certain points of the day (Hirst \& Kalmar, 1987; Necka, 1997). Recent research investigated allocation of 
cognitive resources at the optimal and the non-optimal hours of the day by measuring participants' pupil dilation as they were performing a semantic analogy task (Nowack \& Van Der Meer, 2018). Participants in this study were able to use different resource allocation strategies to partially compensate for circadian-related impairments. These findings suggest that the dropdown in performance at the nonoptimal time of day can be mediated by the type of resource allocation strategies: Participants who are able to avoid wasteful allocation of limited attentional capacities during circadian troughs show lower levels of performance impairments.

The absence of drops in free and cued recall performance in Experiment 1 could be linked to the fact that participants were able to efficiently allocate limited attentional resources. Compared with prior research that relied upon generic memory tests, my experimental protocols provided participants the opportunity to prioritize the limited attentional capacities in the non-optimal sessions efficiently, allowing them to offset the potential impairing effects of circadian troughs. The possibility that the impairing effect of suboptimal factors can be partially compensated by retrieval instructions is an interesting direction for future research. Future experiments can investigate whether the impairing effect of non-optimal testing on performance of mock witnesses materializes when participants are not warned about the subsequent memory test. It is also important to understand whether the negative effects of suboptimal retrieval and identification instructions (Steblay, 1997) can be more strongly pronounced at non-optimal hours.

Identifying the strategies that can be used to counteract the limited availability of cognitive resources offers another interesting avenue for future research. In particular, future studies can use the eyewitness memory paradigm in combination with pupil dilation techniques to measure allocation of cognitive resources in eyewitness memory tasks. It would be interesting to identify the differences in compensatory mechanisms mock witnesses use to offset effects of non-optimal testing. Similar methodology could be used to identify whether encoding and retrieval instructions can encourage the use of efficient compensatory resource allocation strategies when our cognitive capacities are at their low point.

The circadian rhythms and metamemory. My results also outline an interesting direction of future research into time-of-day optimality effects on postdictors of identification accuracy. A traditional approach to studying the way suboptimal factors affect our metamemory judgments is rooted in the optimality hypothesis proposed by Deffenbacher (1980). The general idea behind this theory is that suboptimal factors weaken our metamemory judgments in a similar manner as they impair our memory performance. It follows from this that the predictive value of confidence should be lower in the presence of suboptimal factors at encoding and retrieval. However, an increasing body of evidence suggests that this may not always be the case. The theory-driven confidence judgments hypothesis (Palmer, Brewer, Weber, \& Nagesh, 2013) suggest that people can take into account the presence of impairing factors and adjust their metamemory judgments appropriately. Findings of Experiments 1 and 2 show support for this idea. In Experiment 1, highly confident choosers were significantly better calibrated in their confidence judgments at the non-optimal compared to the optimal time of day. Experiment 2 showed 
that overall confidence-accuracy relationship was stronger (i.e., confidence in choosers was more predictive of accuracy) in the non-optimal compared to the optimal sessions.

The way eyewitnesses perceive troughs in their performance and other factors potentially damaging to their memory in relation to the actual impairment caused by these factors deserves more attention in the eyewitness memory literature. It would be interesting to combine these research questions within a single research line exploring interrelations between the extent to which we are aware of the presence of potentially impairing factors, the compensatory mechanisms we employ to counteract these impairments, and the way this is reflected in our metamemory judgments.

Face recognition and fluctuations in arousal. The current research programme is novel in showing that face recognition performance may not follow the standard synchrony effect patterns. This appears to be a consistent finding in my studies, as evident from identification decisions (Experiments 1 and 2) as well as face recognition data (Experiment 3). To the best of my knowledge, time-of-day effects in memory performance have not been previously tested using facial stimuli.

From a cognitive perspective, processing of faces differs from processing of other types of information (Schwartz, 2014). Faces are processed holistically (e.g., Richler \& Gauthier, 2014) and are distinct in terms of allocation of attention during encoding. For instance, when presented with two faces simultaneously, encoding of one of the faces requires us to suppress processing of features of the other face, whereas this is not the case when we are simultaneously presented with two objects (e.g., Bindemann, Jenkins, \& Burton 2007; Boutet \& Chaudhuri, 2001; Palermo \& Rhodes, 2002). Considering that circadian variations in cognitive performance are generally construed in terms of availability of attentional capacities (Valdez, 2019) and abilities of efficient allocation of cognitive resources (e.g., Nowack \& Van Der Meer, 2018), these peculiarities of face processing may account for the fact that the circadian performance cycles are expressed differently in face recognition performance.

The possibility that attentional mechanisms underlying face processing may offer extra gain in compensating for circadian troughs in cognitive resources outlines an interesting avenue for future research. It is unclear whether face recognition is fully immune to circadian fluctuations in arousal or less sensitive to them. Future studies can investigate this possibility by using an experimental design that includes multiple measurements throughout the day comparing recognition of objects as opposed to faces.

From a neuroscientific perspective, circadian rhythms are not simply a product of oscillations generated in suprachiasmatic nuclei. Areas of our brain responsible for memory functioning appear to show their own autonomous oscillations that can contribute to daily cycles in memory functioning (Snider, Sullivan, \& Obrietan, 2018). In this regard, it may be important to consider that face recognition relies upon specialized areas of the brain different from areas involved in other types of recognition memory. It remains unknown whether the peripheral oscillators in these specialized areas function in a different manner. If this turns out to be the case, face recognition may show a divergent pattern of daily fluctuations in performance compared to other types of stimuli. The combination of brain imaging techniques combined with the strengths of the forced desynchrony protocol may offer promising 
discoveries about dissociations in circadian fluctuations in face recognition performance and recognition of objects and verbal stimuli.

\section{Limitations}

The current study shares its limitations with other studies that rely on the classical synchrony effect paradigm. For instance, the design of my experiments does not allow me to isolate the effect of the circadian rhythm from that of the homeostatic sleep pressure, that is, the decrease in arousal levels associated with the increased amount of time spent awake (Van Dongen \& Dinges, 2003). A demanding paradigm known as the forced desynchrony protocol can allow researchers to overcome this limitation. In this protocol, participants are placed in an environment that isolates them from the external time givers, such as light and social timing cues. This allows to manipulate the sleep and wakefulness cycle in such a way that the duration of the 'day' is other than 24 hours (e.g., 19 hours or 28 hours). As a result, the sleep-wake cycle and the circadian rhythm become desynchronized, allowing researchers to disentangle their complex interactions and measure the effects of each of them separately (Wright, Hull, Hughes, Ronda, \& Czeisler, 2006).

However, these protocols are logistically complicated, considered extreme, and test participants under highly artificial conditions. This undermines the applicability of the findings to the real situations to which eyewitnesses are exposed. Keeping in mind limited resources and the applied focus of the research question, I was not in a position to consider using the forced desynchrony protocol in the current research programme. Nonetheless, this protocol can serve as an excellent methodological platform for disentangling complex interactions of circadian and sleep-related effects on face recognition performance in future studies.

My studies only tested morning- and evening-type individuals, which raises the issue of constraints of generality. Further research into the specifics of time-of-day variations in identification performance in intermediate types is necessary. Comparing memory performance at the optimal and the non-optimal hours in intermediate-type eyewitnesses is important for understanding the variability in performance patterns in the general population. Another limitation of my experiments concerns reliance on self-report tools in determining participants' periods of optimal performance. Future studies may enhance the precision of classifying participants into different chronotype groups by collecting additional physiological measurements, such as cortisol levels and body temperature (Blatter \& Cajochen, 2007).

Finally, encoding and retrieval in all my experiments took place in the same experimental session. This design does not allow us to assess the effects of non-optimal testing on encoding and retrieval differentially. Future studies may address this issue by separating the two memory stages into different testing sessions and manipulating testing optimality for each of them separately, that is, by employing a testing optimality (optimal versus non-optimal) x memory stage (encoding versus retrieval) design. 


\section{Concluding Remarks}

I designed the current research program to answer the applied question of whether circadian arousal could be among the contributors affecting eyewitness memory performance. Across multiple tests, I did not find any empirical evidence supporting the idea that eyewitness memory performance is affected by testing optimality in a similar manner as in other domains of memory performance. This is true for such aspects of eyewitness memory as informativeness and accuracy of free narratives and answers to cued questions, as well as identification performance and ability to discriminate between contexts in which faces were encountered. Therefore, I can conclude that circadian troughs in cognitive performance do not lead to significant reduction of the evidential value of eyewitness testimony in healthy young adults. It is important to emphasize that my findings are limited to situations of optimal encoding and retrieval conditions. Real-life eyewitnesses may encode events under less favourable conditions, such as insufficient lighting (Wagenaar \& van der Schrier, 1996), suboptimal distance (Lindsay, Semmler, Weber, Brewer, \& Lindsay, 2008), or short exposure duration (Memon, Hope, \& Bull, 2003). Moreover, retrieval conditions such as substandard identification instructions (Steblay, 1997) or poor lineup construction (Fitzgerald, Price, Oriet, \& Charman, 2013) increase the likelihood of memory errors. Some of these factors may be more pronounced at circadian arousal troughs, which outlines numerous perspectives for future research. Additionally, elderly eyewitnesses are generally poorer eyewitnesses (see Fitzgerald \& Price, 2015, for a recent meta-analysis). Future studies can test the possibility that obtaining testimony during circadian peaks may partially compensate for this age-related decline in performance in older eyewitnesses. 
Chapter 5 


\section{References}

Adan, A., \& Almirall, H. (1991). Horne \& Östberg morningness-eveningness questionnaire: A reduced scale. Personality and Individual Differences, 12, 241-253. doi:10.1016/0191-8869(91)90110-w

Adan, A., Archer, S., Hidalgo, M., Di Milia, L., Natale, V., \& Randler, C. (2012). Circadian Typology: A Comprehensive Review. Chronobiology International, 29, 1153-1175. doi:10.3109/07420528.2012.719971

Anderson, M., Petros, T., Beckwith, B., Mitchell, W., \& Fritz, S. (1991). Individual differences in the effect of time of day on long-term memory access. The American Journal of Psychology, 104, 241-255. doi:10.2307/1423157

Andrade, M. M., Benedito-Silva, A. A., \& Menna-Barreto, L. (1992). Correlations between morningnesseveningness character, sleep habits and temperature rhythm in adolescents. Brazilian Journal of Medical and Biological Research, 25, 835-839.

Antonenko, D., Diekelmann, S., Olsen, C., Born, J., \& Mölle, M. (2013). Napping to renew learning capacity: Eenhanced encoding after stimulation of sleep slow oscillations. European Journal of Neuroscience, 37, 1142-1151. doi:10.1111/ejn.12118

Atkinson, R. C., \& Juola, J. F. (1974). Search and decision processes in recognition memory. In D. H. Krantz, R. C. Atkinson, R. D. Luce, \& P. Suppes (Eds.), Contemporary developments in mathematical psychology: I. Learning, memory and thinking. W. H. Freeman

Baehr, E. K., Revelle, W., \& Eastman, C. I. (2000). Individual differences in the phase and amplitude of the human circadian temperature rhythm: With an emphasis on morningness-eveningness. Journal of Sleep Research, 9,117-27. doi:10.1046/j.1365-2869.2000.00196.x

Bailey, S.L., \& Heitkemper, M. M. (2001). Circadian rhythmicity of cortisol and body temperature: Morningness-eveningness effects. Chronobiology International, 18, 249-261. doi:10.1081/CBI100103189

Bargh, J. A. (1994). The four horsemen of automaticity: Awareness, intention, efficiency, and control in social cognition. In R. S. Wyer, Jr. \& T. K. Srull (Eds.), Handbook of social cognition: Basic processes; Applications (p. 1-40). Lawrence Erlbaum Associates, Inc

Bargh, J. A., \& Chartrand, T. L. (2000). The mind in the middle: A practical guide to priming and automaticity research. In H. T. Reis \& C. M. Judd (Eds.), Handbook of research methods in social and personality psychology (p. 253-285). Cambridge University Press

Bartlett, F. C. (1932). Remembering: A study in experimental and social psychology. Cambridge University Press.

Benjamin, A. S., \& Craik, F. I. (2001). Parallel effects of aging and time pressure on memory for source: Evidence from the spacing effect. Memory \& Cognition, 29, 691-697. doi:10.3758/BF03200471

Bindemann, M., Jenkins, R., \& Burton, A. M. (2007). A bottleneck in face identification: Repetition priming from flanker images. Experimental Psychology, 54, 192-201. doi:10.1027/1618-

3169.54.3.192 


\section{References}

Blagrove, M., \& Akehurst, L. (2000). Effects of sleep loss on confidence-accuracy relationships for reasoning and eyewitness memory. Journal of Experimental Psychology: Applied, 6, 59-73. doi:10.1037/1076-898x.6.1.59

Blatter, K., \& Cajochen, C. (2007). Circadian rhythms in cognitive performance: Methodological constraints, protocols, theoretical underpinnings. Physiology \& Behavior, 90, 196-208. doi:10.1016/j.physbeh.2006.09.009

Bodenhausen, G. (1990). Stereotypes as judgmental heuristics: Evidence of circadian variations in discrimination. Psychological Science, 1, 319-322. doi:10.1111/j.1467-9280.1990.tb00226.x

Borbély, A., \& Achermann, P. (1999). Sleep homeostasis and models of sleep regulation. Journal of Biological Rhythms, 14, 559-570. doi:10.1177/074873099129000894

Borbély, A., Daan, S., Wirz-Justice, A., \& Deboer, T. (2016). The two-process model of sleep regulation: A reappraisal. Journal of Sleep Research, 25, 131-143. doi:10.1111/jsr.12371

Bousfield, W. A., \& Cohen, B. H. (1953). The effects of reinforcement on the occurrence of clustering in the recall of randomly arranged associates. The Journal of Psychology, 36, 67-81. doi:10.1080/00223980.1953.9712878

Boutet, I., \& Chaudhuri, A. (2001). Multistability of overlapped face stimuli is dependent upon orientation. Perception, 30, 743-753. doi:10.1068/p3183

Brackmann, N., Sauerland, M., \& Otgaar, H. (2019). Developmental trends in lineup performance: Adolescents are more prone to innocent bystander misidentifications than children and adults. Memory \& Cognition, 47, 428-440. doi:10.3758/s13421-018-0877-6

Bradfield, A., \& Wells, G. (2000). The perceived validity of eyewitness identification testimony: A test of the five Biggers criteria. Law and Human Behavior, 24, 581-594. doi:10.1023/a:1005523129437

Brewer, N., \& Wells, G. (2006). The confidence-accuracy relationship in eyewitness identification: Effects of lineup instructions, foil similarity, and target-absent base rates. Journal of Experimental Psychology: Applied, 12, 11-30. doi:10.1037/1076-898x.12.1.11

Brewer, N., \& Wells, G. (2006). The confidence-accuracy relationship in eyewitness identification: Effects of lineup instructions, foil similarity, and target-absent base rates. Journal of Experimental Psychology: Applied, 12, 11-30. doi:10.1037/1076-898x.12.1.11

Brewer, N., Vagadia, A. N., Hope, L., \& Gabbert, F. (2018). Interviewing witnesses: Eliciting coarse-grain information. Law and Human Behavior, 42, 458-471. doi:10.1037/Ihb0000294

Brown, E., Deffenbacher, K., \& Sturgill, W. (1977). Memory for faces and the circumstances of encounter. Journal of Applied Psychology, 62, 311-318. doi:10.1037/0021-9010.62.3.311

Bruce, V., \& Young, A. (2012). Face perception. New York, NY: Psychology Press. doi:10.4324/9780203721254

Buhrmester, M., Kwang, T., \& Gosling, S. (2011). Amazon's Mechanical Turk: A new source of inexpensive, yet high-quality, data? Perspectives on Psychological Science, 6, 3-5. doi:10.1177/1745691610393980 
Burton, A.M. (2013). Why has research in face recognition progressed so slowly? The importance of variability. Quarterly Journal of Experimental Psychology, 66, 1467-1485.

doi:10.1080/17470218.2013.800125

Buysse, D., Reynolds, C., Monk, T., Berman, S., \& Kupfer, D. (1989). The Pittsburgh sleep quality index: A new instrument for psychiatric practice and research. Psychiatry Research, 28, 193-213. doi:10.1016/0165-1781(89)90047-4

Caci, H., Deschaux, O., Adan, A., \& Natale, V. (2009). Comparing three morningness scales: Age and gender effects, structure and cut-off criteria. Sleep Medicine, 10, 240-245. doi:10.1016/j.sleep.2008.01.007

Cann, D., McRae, K., \& Katz, A. (2011). False recall in the Deese-Roediger-McDermott paradigm: The roles of gist and associative strength. Quarterly Journal of Experimental Psychology, 64, 15151542. doi:10.1080/17470218.2011.560272

Cariou, M., Galy, E., \& Mélan, C. (2008). Differential 24-hour variation of alertness and subjective tension in process controllers: Investigation of the relationship with body temperature and heart rate. Chronobiology International, 25, 597-609. doi:10.1080/07420520802261838

Chellappa, S., Morris, C., \& Scheer, F. (2018). Daily circadian misalignment impairs human cognitive performance task-dependently. Scientific Reports, 8, 1-11. doi:10.1038/s41598-018-20707-4

Chen, H., Cohen, P., \& Chen, S. (2010). How big is a big odds ratio? Interpreting the magnitudes of odds ratios in epidemiological studies. Communications in Statistics - Simulation and Computation, 39, 860-864. doi:10.1080/03610911003650383

Chun, M., \& Turk-Browne, N. (2007). Interactions between attention and memory. Current Opinion in Neurobiology, 17, 177-184. doi:10.1016/j.conb.2007.03.005

Clark, S., Benjamin, A., Wixted, J., Mickes, L., \& Gronlund, S. (2015). Eyewitness identification and the accuracy of the criminal justice system. Policy Insights from the Behavioral and Brain Sciences, 2, 175-186. doi:10.1177/2372732215602267

Cousins, J., Sasmita, K., \& Chee, M. (2017). Memory encoding is impaired after multiple nights of partial sleep restriction. Journal of Sleep Research, 27, 138-145. doi:10.1111/jsr.12578

Craik, F. I. M. (2002). Levels of processing: Past, present... and future? Memory, 10, 305-318. doi:10.1080/09658210244000135

Craik, F. I. M. (1999). Levels of encoding and retrieval. In: Challis, B. H., Velichkovskii, B. H. (Eds.). Stratification in Cognition and Consciousness (pp. 97-104). Amsterdam: John Benjamins. doi:10.1075/aicr.15.09cra

Craik, F. I., \& Lockhart, R. S. (1972). Levels of processing: A framework for memory research. Journal of verbal learning and verbal behaviour, 11, 671-684. doi:1016/S0022-5371(72)80001-X

Craik, F. I., \& Tulving, E. (1975). Depth of processing and the retention of words in episodic memory. Journal of Experimental Psychology: General, 104, 268-294. doi:10.1037//00963445.104.3.268 


\section{References}

Craik, F. I., Govoni, R., Naveh-Benjamin, M., \& Anderson, N. D. (1996). The effects of divided attention on encoding and retrieval processes in human memory. Journal of Experimental Psychology: General, 125, 159-180. doi:10.1037/0096-3445.125.2.159

Crump, M., McDonnell, J., \& Gureckis, T. (2013). Evaluating Amazon's Mechanical Turk as a tool for experimental behavioural research. Plos ONE, 8, e57410. doi:10.1371/journal.pone.0057410

Czeisler, C., \& Gooley, J. (2007). Sleep and circadian rhythms in humans. Cold Spring Harbor Symposia on Quantitative Biology, 72, 579-597. doi:10.1101/sqb.2007.72.064

Deese, J. (1959). On the prediction of occurrence of particular verbal intrusions in immediate recall. Journal of Experimental Psychology, 58, 17-22. doi:10.1037/h0046671

Deffenbacher, K. (1980). Eyewitness accuracy and confidence: Can we infer anything about their relationship? Law and Human Behavior, 4, 243-260. doi:10.1007/bf01040617

Deffenbacher, K., Bornstein, B., \& Penrod, S. (2006). Mugshot exposure effects: Retroactive interference, mugshot commitment, source confusion, and unconscious transference. Law and Human Behavior, 30, 287-307. doi:10.1007/s10979-006-9008-1

Diges, M., Rubio, M.E., \& Rodriguez, C. (1992). Eyewitness memory and time of day. In F. Losel, D. Bender, \& T. Bliesener (Eds.), Psychology and Law. International Perspectives (pp. 317-320). Berlin: W. De Gruyter.

Di Milia, L., Adan, A., Natale, V., \& Randler, C. (2013). Reviewing the psychometric properties of contemporary circadian typology measures. Chronobiology International, 30, 1261-1271. doi:10.3109/07420528.2013.817415

Duffy, J. F., Rimmer, D. W., \& Czeisler, C. A. (2001). Association of intrinsic circadian period with morningness-eveningness, usual wake time, and circadian phase. Behavioural Neuroscience, 115, 895-899. doi:10.1037/0735-7044.115.4.895

Dunning, D., \& Stern, L. (1994). Distinguishing accurate from inaccurate eyewitness identifications via inquiries about decision processes. Journal of Personality and Social Psychology, 67, 818-835. doi:10.1037//0022-3514.67.5.818

Eckel-Mahan, K., \& Storm, D. (2009). Circadian rhythms and memory: not so simple as cogs and gears. EMBO Reports, 10, 584-591. doi:10.1038/embor.2009.123

Fabbri, M., Antonietti, A., Giorgetti, M., Tonetti, L., \& Natale, V. (2007). Circadian typology and style of thinking differences. Learning and Individual Differences, 17, 175-180. doi:10.1016/j.lindif.2007.05.002

Faul, F., Erdfelder, E., Buchner, A., \& Lang, A. (2009). Statistical power analyses using G*Power 3.1: Tests for correlation and regression analyses. Behavior Research Methods, 41, 1149-1160. doi:10.3758/brm.41.4.1149

Faul, F., Erdfelder, E., Lang, A., \& Buchner, A. (2007). G*Power 3: A flexible statistical power analysis program for the social, behavioral, and biomedical sciences. Behavior Research Methods, 39 , 175-191. doi:10.3758/bf03193146 
Felson, M., \& Poulsen, E. (2003). Simple indicators of crime by time of day. International Journal of Forecasting, 19, 595-601. doi:10.1016/s0169-2070(03)00093-1

Fernandes, M. A., \& Moscovitch, M. (2000). Divided attention and memory: evidence of substantial interference effects at retrieval and encoding. Journal of Experimental Psychology: General, 129, 155-176. doi:10.1037//0096-3445.129.2.155

Fisk, A., Tam, S., Brown, L., Vyazovskiy, V., Bannerman, D., \& Peirson, S. (2018). Light and cognition: roles for circadian rhythms, sleep, and arousal. Frontiers in Neurology, 9. doi:10.3389/fneur.2018.00056

Fitzgerald, R. J., Price, H. L., Oriet, C., \& Charman, S. D. (2013). The effect of suspect-filler similarity on eyewitness identification decisions: A meta-analysis. Psychology, Public Policy, and Law, 19, 151-164. doi:10.1037/a0030618

Fitzgerald, R., \& Price, H. (2015). Eyewitness identification across the life span: A meta-analysis of age differences. Psychological Bulletin, 141, 1228-1265. doi:10.1037/bul0000013

Fleischer, A., Mead, A., \& Huang, J. (2015). Inattentive responding in MTurk and other online samples. Industrial and Organizational Psychology, 8, 196-202. doi:10.1017/iop.2015.25

Flowe, H., Colloff, M., Karoğlu, N., Zelek, K., Ryder, H., Humphries, J., \& Takarangi, M. (2017). The effects of alcohol intoxication on accuracy and the confidence-accuracy relationship in photographic simultaneous line-ups. Applied Cognitive Psychology, 31, 379-391. doi:10.1002/acp.3332

Forst, B. (2013). Wrongful convictions in a world of miscarriages of justice. In C.R. Huff and M. Killias (Eds.), Wrongful Convictions and Miscarriages of Justice: Causes and remedies in North American and European criminal justice systems (pp. 15-43). London: Routledge.

Gabbert, F., Hope, L., \& Confrey, M. (2018). Witness testimony. In A. Griffiths, \& R. Milne (Eds.), The Psychology of Criminal Investigation: From Theory to Practice. Routledge.

Gaggioni, G., Maquet, P., Schmidt, C., Dijk, D., \& Vandewalle, G. (2014). Neuroimaging, cognition, light and circadian rhythms. Frontiers in Systems Neuroscience, 8, 1-12. doi:10.3389/fnsys.2014.00126

Glass, L. (2001). Synchronization and rhythmic processes in physiology. Nature, 410, 277-284. doi:10.1038/35065745

Goldstein, D., Hahn, C. S., Hasher, L., Wiprzycka, U. J., \& Zelazo, P. D. (2007). Time of day, intellectual performance, and behavioral problems in Morning versus Evening type adolescents: Is there a synchrony effect? Personality and Individual Differences, 42, 431-440. doi:10.1016/j.paid.2006.07.008

Goodsell, C. A., Neuschatz, J. S., \& Gronlund, S. D. (2009). Effects of mugshot commitment on lineup performance in young and older adults. Applied Cognitive Psychology, 23, 788-803. doi:10.1002/acp.1512

Green, D., \& Swets, J. (1966). Signal detection theory and psychophysics. New York: Wiley. 


\section{References}

Halberg, F., Cornélissen, G., Katinas, G., Syutkina, E. V., Sothern, R. B., Zaslavskaya, R., ... \& Tarquini, R. (2003). Transdisciplinary unifying implications of circadian findings in the 1950s. Journal of circadian rhythms, 1, 2.

Hasher, L., \& Zacks, R. (1984). Automatic processing of fundamental information: The case of frequency of occurrence. American Psychologist, 39, 1372-1388. doi:10.1037/0003-066x.39.12.1372

Hasher, L., \& Zacks, R. T. (1979). Automatic and effortful processes in memory. Journal of Experimental Psychology: General, 108, 356-388. doi:10.1037/0096-3445.108.3.356

Hastie, R., \& Kumar, P. A. (1979). Person memory: Personality traits as organizing principles in memory for behaviors. Journal of Personality and Social Psychology, 37, 25-38. doi:10.1037/00223514.37.1.25

Hastings, M., Reddy, A., \& Maywood, E. (2003). A clockwork web: Circadian timing in brain and periphery, in health and disease. Nature Reviews Neuroscience, 4, 649-661. doi:10.1038/nrn1177

Haxby, J. V., Gobbini, M. I., Furey, M. L., Ishai, A., Schouten, J. L., \& Pietrini, P. (2001). Distributed and overlapping representations of faces and objects in ventral temporal cortex. Science, 293, 2425-2430. doi:10.1126/science.1063736

Haxby, J., Hoffman, E., \& Gobbini, M. (2002). Human neural systems for face recognition and social communication. Biological Psychiatry, 51, 59-67. doi:10.1016/s0006-3223(01)01330-0

Hirst, W., \& Kalmar, D. (1987). Characterizing attentional resources. Journal of Experimental Psychology: General, 116, 68-81. doi:10.1037/0096-3445.116.1.68

Horne, J. A., \& Östberg, O. (1976). A self-assessment questionnaire to determine morningnesseveningness in human circadian rhythms. International Journal of Chronobiology, 4, 97-100. doi:10.1177/0748730405285278

Ingram, K., Ay, A., Kwon, S., Woods, K., Escobar, S., \& Gordon, M., ... Jain, K. (2016). Molecular insights into chronotype and time-of-day effects on decision-making. Scientific Reports, 6, 1-9. doi:10.1038/srep29392

Innocence Project. (2020). http://www.innocenceproject.org/Last visited May 10, 2020.

Intons-Peterson, M. J., Rocchi, P., West, T., McLellan, K., \& Hackney, A. (1999). Age, testing at preferred or nonpreferred times (testing optimality), and false memory. Journal of Experimental Psychology: Learning, Memory, and Cognition, 25, 23-40. doi:10.1037/0278-7393.25.1.23 $10.2307 / 2531734$

Jacoby, L. L. (1998). Invariance in automatic influences of memory: Toward a user's guide for the process-dissociation procedure. Journal of Experimental Psychology: Learning, Memory, and Cognition, 24, 3-26. doi:10.1037/0278-7393.24.1.3

Jacoby, L. L., \& Dallas, M. (1981). On the relationship between autobiographical memory and perceptual learning. Journal of Experimental Psychology: General, 110, 306340. doi:10.1037/0096-3445.110.3.306 
Jacoby, L. L., Woloshyn, V., \& Kelley, C. M. (1989). Becoming famous without being recognized: Unconscious influences of memory produced by dividing attention. Journal of Experimental Psychology: General, 118, 115-125. doi:10.1037/0096-3445.118.2.115

Jeffreys, H. (1967). Theory of probability. Oxford: Clarendon Press.

Johnson, M. K., Hashtroudi, S., \& Lindsay, D. S. (1993). Source monitoring. Psychological Bulletin, 114, 3-28. doi:10.1037/0033-2909.114.1.3

Kahneman, D. (1973). Attention and effort. Englewood Cliffs, NJ: Prentice-Hall.

Kane, M. J., \& Engle, R. W. (2000). Working-memory capacity, proactive interference, and divided attention: Limits on long-term memory retrieval. Journal of Experimental Psychology: Learning, Memory, and Cognition, 26, 336-358. doi:10.1037//0278-7393.26.2.336

Kanwisher, N., McDermott, J., \& Chun, M. (1997). The fusiform face area: A module in human extrastriate cortex specialized for face perception. The Journal of Neuroscience, 17, 4302-4311. doi:10.1523/jneurosci.17-11-04302.1997

Kebbell, M. R., \& Milne, R. (1998). Police officers' perceptions of eyewitness performance in forensic investigations. The Journal of Social Psychology, 138, 323-330. doi:10.1080/00224549809600384

Kelley, P., Lockley, S., Foster, R., \& Kelley, J. (2014). Synchronizing education to adolescent biology: 'Let teens sleep, start school later'. Learning, Media and Technology, 40, 210-226. doi:10.1080/17439884.2014.942666

Kihlstrom, J. (2009). 'So that we might have roses in December': The functions of autobiographical memory. Applied Cognitive Psychology, 23, 1179-1192. doi:10.1002/acp.1618

Kihlstrom, J. F. (1996). Memory research: The convergence of theory and practice. In D. Hermann, M. Johnson, C. McEvoy, C. Hertzog, \& P. Hertel (Eds.), Basic and applied memory: Theory in context (Vol. 1, pp. 5-25). Mahwah, NJ: Erlbaum

Krishnan, H., \& Lyons, L. (2015). Synchrony and desynchrony in circadian clocks: Impacts on learning and memory. Learning \& Memory, 22, 426-437. doi:10.1101/Im.038877.115

Kyriacou, C., \& Hastings, M. (2010). Circadian clocks: Genes, sleep, and cognition. Trends in Cognitive Sciences, 14, 259-267. doi:10.1016/j.tics.2010.03.007

Lee, M. D., \& Wagenmakers, E.-J. (2013). Bayesian cognitive modeling: A practical course. Cambridge University Press. doi:10.1017/CBO9781139087759

Levandovski, R., Sasso, E., \& Hidalgo, M. (2013). Chronotype: A review of the advances, limits and applicability of the main instruments used in the literature to assess human phenotype. Trends in Psychiatry and Psychotherapy, 35, 3-11. doi:10.1590/s2237-60892013000100002

Lockhart, R. S. (1992). Levels of processing. In: L. R. Squire (Ed.), Encyclopedia of Learning and Memory. New York, NY: Macmillan.

Loftus, E. F. (1975). Leading questions and the eyewitness report. Cognitive Psychology, 7, 560-572. doi:10.1016/0010-0285(75)90023-7 


\section{References}

Loftus, E. F. (2005). Planting misinformation in the human mind: A 30-year investigation of the malleability of memory. Learning \& Memory, 12, 361-366. doi:10.1101/Im.94705

Loftus, E. F., \& Palmer, J. C. (1974). Reconstruction of automobile destruction: An example of the interaction between language and memory. Journal of Verbal Learning and Verbal Behavior, 13, 585-589. doi:10.1016/S0022-5371(74)80011-3

Loftus, E. F., Miller, D. G., \& Burns, H. J. (1978). Semantic integration of verbal information into a visual memory. Journal of Experimental Psychology: Human Learning and Memory, 4, 1931. doi:10.1037/0278-7393.4.1.19

Loftus, E. F. (2003). Our changeable memories: Legal and practical implications. Nature Reviews: Neuroscience, 4, 231-234. doi:10.1038/nrn1054

Lundqvist, D., Flykt, A., \& Öhman, A. (1998). The Karolinska Directed Emotional Faces - KDEF, CD ROM from Department of Clinical Neuroscience, Psychology section, Karolinska Institutet. ISBN 91630-7164-9.

MacLin, O., MacLin, M., \& Malpass, R. (2001). Race, arousal, attention, exposure and delay: An examination of factors moderating face recognition. Psychology, Public Policy, and Law, 7, 134152. doi:10.1037/1076-8971.7.1.134

Madon, S., Guyll, M., Scherr, K., Greathouse, S., \& Wells, G. (2012). Temporal discounting: The differential effect of proximal and distal consequences on confession decisions. Law and Human Behavior, 36, 13-20. doi:10.1037/h0093962

Mandler, G. (1980). Recognizing: The judgment of previous occurrence. Psychological Review, 87, 252271. doi:10.1037//0033-295x.87.3.252

Mason, W., \& Suri, S. (2011). Conducting behavioral research on Amazon's Mechanical Turk. Behavior Research Methods, 44, 1-23. doi:10.3758/s13428-011-0124-6

May, C. (1999). Synchrony effects in cognition: The costs and a benefit. Psychonomic Bulletin \& Review, 6, 142-147. doi:10.3758/bf03210822

May, C. P., Hasher, L., \& Foong, N. (2005). Implicit memory, age, and time of day: Paradoxical priming effects. Psychological Science, 16, 96-100. doi:10.1111/j.0956-7976.2005.00788.x

May, C. P., Hasher, L., \& Stoltzfus, E. R. (1993). Optimal time of day and the magnitude of age differences in memory. Psychological Science, 4, 326-330. doi:10.1111/j.14679280.1993.tb00573.x

May, C., \& Hasher, L. (1998). Synchrony effects in inhibitory control over thought and action. Journal of Experimental Psychology: Human Perception and Performance, 24, 363-379. doi:10.1037//0096-1523.24.2.363

Meade, A., \& Craig, S. (2012). Identifying careless responses in survey data. Psychological Methods, 17, 437-455. doi:10.1037/a0028085

Meissner, C., \& Brigham, J. (2001). A meta-analysis of the verbal overshadowing effect in face identification. Applied Cognitive Psychology, 15, 603-616. doi:10.1002/acp.728 
Meissner, C., Tredoux, C., Parker, J., \& MacLin, O. (2005). Eyewitness decisions in simultaneous and sequential lineups: A dual-process signal detection theory analysis. Memory \& Cognition, 33, 783-792. doi:10.3758/bf03193074

Memon, A., Hope, L., \& Bull, R. (2003). Exposure duration: Effects on eyewitness accuracy and confidence. British Journal of Psychology, 94, 339-354. doi:10.1348/000712603767876262

Memon, A., Hope, L., Bartlett, J., \& Bull, R. (2002). Eyewitness recognition errors: The effects of mugshot viewing and choosing in young and old adults. Memory \& Cognition, 30, 1219-1227. doi:10.3758/bf03213404

Mitchell, K. J., \& Johnson, M. K. (2009). Source monitoring 15 years later: What have we learned from fMRI about the neural mechanisms of source memory? Psychological Bulletin, 135, 638677. doi:10.1037/a0015849

Monk, T. (1992). Circardian rhythms in subjective activation, mood, and performance efficiency. In M. Kryger, T. Roth, \& W. Dement (Eds.). Principles and practice of sleep medicine, 2nd Ed. (pp. 321330). Philadelphia: WB Saunders.

Morgan, D., Tamminen, J., Seale-Carlisle, T., \& Mickes, L. (2019). The impact of sleep on eyewitness identifications. Royal Society Open Science, 6, 170501. doi:10.1098/rsos.170501

Moritz, S., Woodward, T. S., \& Rodriguez-Raecke, R. (2006). Patients with schizophrenia do not produce more false memories than controls but are more confident in them. Psychological Medicine, 36, 659-667. doi:10.1017/S0033291706007252

Mulligan, N. W. (1998). The role of attention during encoding in implicit and explicit memory. Journal of Experimental Psychology: Learning, Memory, and Cognition, 24, 27. doi:10.1037/02787393.24.1.27

Natale, V., \& Cicogna, P. (2002). Morningness-eveningness dimension: Is it really a continuum? Personality and Individual Differences, 32, 809-816. doi:10.1016/S01918869(01)00085-X

Natale, V., Esposito, M., Martoni, M., \& Fabbri, M. (2006). Validity of the reduced version of the Morningness-Eveningness Questionnaire. Sleep and Biological Rhythms, 4, 72-74. doi:10.1111/j.1479-8425.2006.00192.x

Necka, E. (1997). Attention, working memory and arousal: Concepts apt to account for the "process of intelligence". In G. Matthews (Ed.), Cognitive Science Perspectives on Personality and Emotion (pp. 503-554). North-Holland: Elsevier. doi:10.1016/S0166-4115(97)80129-X

Nowack, K., \& Van Der Meer, E. (2018). Nowack, K., Meer, E. Van Der, \& Nowack, K. (2018). The synchrony effect revisited: Chronotype, time of day and cognitive performance in a semantic analogy task performance in a semantic analogy task. Chronobiology International, 36, 16471662. doi:10.1080/07420528.2018.1500477

Oppenheimer, D. M., Meyvis, T., \& Davidenko, N. (2009). Instructional manipulation checks: Detecting satisficing to increase statistical power. Journal of Experimental Social Psychology, 45, 867-872. 


\section{References}

Otgaar, H., Howe, M. L., Brackmann, N., \& Smeets, T. (2016). The malleability of developmental trends in neutral and negative memory illusions. Journal of Experimental Psychology: General, 145, 31 55. doi:10.1037/xge0000127

Otgaar, H., Howe, M. L., Peters, M., Smeets, T., \& Moritz, S. (2014). The production of spontaneous false memories across childhood. Journal of Experimental Child Psychology, 121, 2841. doi.org:10.1016/j.jecp.2013.11.019

Otten, L. J., Henson, R. N., \& Rugg, M. D. (2001). Depth of processing effects on neural correlates of memory encoding: Relationship between findings from across- and within-task comparisons. Brain: A Journal of Neurology, 124, 399-412. doi:10.1093/brain/124.2.399

Palermo, R., \& Rhodes, G. (2002). The influence of divided attention on holistic face perception. Cognition, 82, 225-257. doi:10.1016/S0010-0277(01)00160-3

Palmer, M. A., Brewer, N., Weber, N., \& Nagesh, A. (2013). The confidence-accuracy relationship for eyewitness identification decisions: Effects of exposure duration, retention interval, and divided attention. Journal of Experimental Psychology: Applied, 19, 55-71. doi:10.1037/a0031602

Paolacci, G., \& Chandler, J. (2014). Inside the Turk. Current Directions in Psychological Science, 23, $184-$ 188. doi:10.1177/0963721414531598

Paolacci, G., Chandler, J., \& Ipeirotis, P. G. (2010). Running experiments on Amazon Mechanical Turk. Judgment and Decision Making, 5, 411-419.

Payne, J., Schacter, D., Propper, R., Huang, L., Wamsley, E., \& Tucker, M. et al. (2009). The role of sleep in false memory formation. Neurobiology Of Learning And Memory, 92, 327-334. doi: 10.1016/j.nIm.2009.03.007

Peek, C., Levine, D., Cedernaes, J., Taguchi, A., Kobayashi, Y., \& Tsai, S. et al. (2017). Circadian clock interaction with HIF1 $\alpha$ mediates oxygenic metabolism and anaerobic glycolysis in skeletal muscle. Cell Metabolism, 25, 86-92. doi:10.1016/j.cmet.2016.09.010

Perfect, T.J., \& Harris, L.J. (2003) Adult age differences in unconscious transference: Source confusion or identity blending? Memory \& Cognition 31, 570-580. doi:10.3758/BF03196098

Peters, M., Engel, M., Hauschildt, M., Moritz, S., Jelinek, L., \& Otgaar, H. (2012). Investigating the corrective effect of forewarning on memory and meta-memory deficits in schizophrenia patients. Journal of Experimental Psychopathology, 3, 673-687. doi:10.5127/jep.022011

Peterson, J., Sommers, I., Baskin, D., \& Johnson, D. (2010). The role and impact offorensic evidence in the criminal justice process. Revised final report. Washington, DC: National Institute of Justice.

Petros, T., Beckwith, B., \& Anderson, M. (1990). Individual differences in the effects of time of day and passage difficulty on prose memory in adults. British Journal of Psychology, 81, 63-72. doi:10.1111/j.2044-8295.1990.tb02346.x

Puttaert, D., Adam, S., \& Peigneux, P. (2018). Subjectively-defined optimal / non-optimal time of day modulates controlled but not automatic retrieval processes in verbal memory. Journal of Sleep Research, 28, e12798. doi:10.1111/jsr.12798 
Rand, D. (2012). The promise of Mechanical Turk: How online labor markets can help theorists run behavioral experiments. Journal of Theoretical Biology, 299, 172-179.

doi:10.1016/j.jtbi.2011.03.004

Rasch, B., \& Born, J. (2013). About sleep's role in memory. Physiological Reviews, 93, 681-766. doi:10.1152/physrev.00032.2012

Read, J. D., Tollestrup, P., Hammersley, R., McFadzen, E., \& Christensen, A. (1990). The unconscious transference effect: Are innocent bystanders ever misidentified? Applied Cognitive Psychology, 4, 3-31. doi:10.1002/acp.2350040103

Refinetti, R. (2006). Circadian physiology (2nd ed.). CRC Press.

Reppert, S. M., \& Weaver, D. R. (2002). Coordination of circadian timing in mammals. Nature, 418, 935941. doi:10.1038/nature00965

Richler, J. J., \& Gauthier, I. (2014). A meta-analysis and review of holistic face processing. Psychological Bulletin, 140, 1281-1302. doi:10.1037/a0037004

Ridley, A. M., Gabbert, F., \& La Rooy, D. J. (Eds.). (2013). Wiley series in the psychology of crime, policing and law. Suggestibility in legal contexts: Psychological research and forensic implications. WileyBlackwell.

Riley, E., Esterman, M., Fortenbaugh, F., \& DeGutis, J. (2017). Time-of-day variation in sustained attentional control. Chronobiology International, 34, 993-1001. doi:10.1080/07420528.2017.1308951

Robotham, R. J., \& Starrfelt, R. (2017). Face and word recognition can be selectively affected by brain injury or developmental disorders. Frontiers in Psychology, 8, 1547. doi:10.3389/fpsyg.2017.01547

Roediger, H., \& McDermott, K. (1995). Creating false memories: Remembering words not presented in lists. Journal of Experimental Psychology: Learning, Memory, and Cognition, 21, 803-814. doi:10.1037//0278-7393.21.4.803

Roediger, H., Watson, J., McDermott, K., \& Gallo, D. (2001). Factors that determine false recall: A multiple regression analysis. Psychonomic Bulletin \& Review, 8, 385-407. doi:10.3758/bf03196177

Roenneberg, T., Kuehnle, T., Juda, M., Kantermann, T., Allebrandt, K., Gordijn, M., \& Merrow, M. (2007). Epidemiology of the human circadian clock. Sleep Medicine Reviews, 11, 429438. doi:10.1016/j.smrv.2007.07.005

Roenneberg, T., Wirz-Justice, A., \& Merrow, M. (2003). Life between clocks: Daily temporal patterns of human chronotypes. Journal of Biological Rhythms, 18, 80-90. doi:10.1177/0748730402239679

Ross, D. R., Ceci, S. J., Dunning, D., \& Toglia, M. P. (1994). Unconscious transference and mistaken identity: When a witness misidentifies a familiar but innocent person. Journal of Applied Psychology, 79, 918-930. doi:10.1037/0021-9010.79.6.918

Ryan, L., Hatfield, C., \& Hofstetter, M. (2002). Caffeine reduces time-of-day effects on memory performance in older adults. Psychological Science, 13, 68-71. doi:10.1111/1467-9280.00412 


\section{References}

Sauer, J., \& Hope, L. (2016). The effects of divided attention at study and reporting procedure on regulation and monitoring for episodic recall. Acta Psychologica, 169, 143-156. doi:10.1016/j.actpsy.2016.05.015

Sauerland, M., \& Sporer, S. (2007). Post-decision confidence, decision time, and self-reported decision processes as postdictors of identification accuracy. Psychology, Crime \& Law, 13, 611-625. doi:10.1080/10683160701264561

Sauerland, M., \& Sporer, S. L. (2009). Fast and confident: Postdicting eyewitness identification accuracy in a field study. Journal of Experimental Psychology: Applied, 15, 46-62. doi:10.1037/a0014560

Sauerland, M., Krix, A., van Kan, N., Glunz, S., \& Sak, A. (2014). Speaking is silver, writing is golden? The role of cognitive and social factors in written vs. spoken witness accounts. Memory \& Cognition, 42, 978-992. doi:10.3758/s13421-014-0401-6

Schacter, D. L., Kaszniak, A. W., Kihlstrom, J. F., \& Valdiserri, M. (1991). The relation between source memory and aging. Psychology and Aging, 6, 559-568. doi:10.1037/0882-7974.6.4.559

Scherr, K., Miller, J., \& Kassin, S. (2014). "Midnight confessions": The effect of chronotype asynchrony on admissions of wrongdoing. Basic and Applied Social Psychology, 36, 321-328. doi:10.1080/01973533.2014.917974

Schmidt, C., Collette, F., Cajochen, C., \& Peigneux, P. (2007). A time to think: Circadian rhythms in human cognition. Cognitive Neuropsychology, 24, 755-789. doi:10.1080/02643290701754158

Schoenfield, D., and Stones, M. J. (1979). Remembering and aging. In Kihlstrom, J. F., and Evans, F. J. (Eds.), Functional Disorders of Memory. Erlbaum, Hillsdale, New Jersey

Schonfield, D., \& Robertson, B. A. (1966). Memory storage and aging. Canadian Journal of Psychology/Revue canadienne de psychologie, 20, 228-236. doi:10.1037/h0082941

Schwartz, B. L. (2014). Memory for people: Integration of face, voice, name, and biographical information. In T. J. Perfect \& D. S. Lindsay (Eds.), The SAGE Handbook of Applied Memory (pp. 3-19). London, UK: SAGE. doi:10.4135/9781446294703.n1

Sim, J., \& Wright, C. (2005). The kappa statistic in reliability studies: Use, interpretation, and sample size requirements. Physical Therapy, 85, 257-268. doi:10.1093/ptj/85.3.257

Smith, C., Reilly, C., \& Midkiff, K. (1989). Evaluation of three circadian rhythm questionnaires with suggestions for an improved measure of morningness. Journal of Applied Psychology, 74, 728738. doi:10.1037//0021-9010.74.5.728

Smith, E. R., \& DeCoster, J. (2000). Dual-process models in social and cognitive psychology: Conceptual integration and links to underlying memory systems. Personality and Social Psychology Review, 4, 108-131. doi:10.1207/S15327957PSPR0402_01

Smolensky, M. H., \& Peppas, N. A. (2007). Chronobiology, drug delivery, and chronotherapeutics. Advanced Drug Delivery Reviews, 59, 828-851.

Snider, K., Sullivan, K., \& Obrietan, K. (2018). Circadian regulation of hippocampal-dependent emory: Circuits, synapses, and molecular mechanisms. Neural Plasticity, 2018, 1-13. doi:10.1155/2018/7292540 
Snodgrass, J. G., \& Corwin, J. (1988). Pragmatics of measuring recognition memory: Applications to dementia and amnesia. Journal of Experimental Psychology: General, 117, 34-

50. doi:10.1037/0096-3445.117.1.34

Sporer, S., Penrod, S., Read, D., \& Cutler, B. (1995). Choosing, confidence, and accuracy: A metaanalysis of the confidence-accuracy relation in eyewitness identification studies. Psychological Bulletin, 118, 315-327. doi:10.1037/0033-2909.118.3.315

Steblay, N. M. (1992). A meta-analytic review of the weapon focus effect. Law and Human Behavior, 16, 413-424. doi:0.1007/BF02352267

Steblay, N. (1997). Social influence in eyewitness recall: A meta-analytic review of lineup instruction effects. Law and Human Behavior, 21, 283-297. doi:10.1023/a:1024890732059

Stepan, M., Dehnke, T., \& Fenn, K. (2017). Sleep and eyewitness memory: Fewer false identifications after sleep when the target is absent from the lineup. PLOS ONE, 12, e0182907. doi:10.1371/journal.pone.0182907

St-Yves, M. (2015). Investigative Interviewing: The Essential Handbook of Best Practices. Canada: Carswell.

Tanaka, J., \& Farah, M. (1993). Parts and wholes in face recognition. The Quarterly Journal of Experimental Psychology Section A, 46, 225-245. doi:10.1080/14640749308401045

Thayer, R. (1987). Problem perception, optimism, and related states as a function of time of day (diurnal rhythm) and moderate exercise: Two arousal systems in interaction. Motivation and Emotion, 11, 19-36. doi:10.1007/bf00992211

Torsvall, L., \& Åkerstedt, T. (1980). A diurnal type scale. Construction, consistency and validation in shift work. Scandinavian Journal of Work, Environment \& Health, 6, 283-290. doi:10.5271/sjweh.2608

Tredoux, C. (1999). Statistical considerations when determining measures of lineup size and lineup bias. Applied Cognitive Psychology, 13, 9-26. doi:10.1002/(SICI)1099-0720

Trott, C. T., Friedman, D., Ritter, W., Fabiani, M., \& Snodgrass, J. G. (1999). Episodic priming and memory for temporal source: Event-related potentials reveal age-related differences in prefrontal functioning. Psychology and Aging, 14, 390-413. doi:10.1037/0882-7974.14.3.390

Tulving, E. (1962). Subjective organization in free recall of" unrelated" words. Psychological review, 69, 344-354. doi:10.1037/h0043150

Valdez P. (2019). Circadian rhythms in attention. The Yale Journal of Biology and Medicine, 92, 81-92.

Valdez, P. (2019). Homeostatic and circadian regulation of cognitive performance. Biological Rhythm Research, 50, 85-93. doi:10.1080/09291016.2018.1491271

Van Dongen H., \& Dinges D. F. (2003). Investigating the interaction between the homeostatic and circadian processes of sleep-wake regulation for the prediction of waking neurobehavioural performance. Journal of Sleep Research, 12, 181-187. doi:10.1046/j.1365-2869.2003.00357.x

van Kesteren, M. T. R., Ruiter, D. J., Fernández, G., \& Henson, R. N. (2012). How schema and novelty augment memory formation. Trends in Neurosciences, 35, 211-

219. doi:10.1016/j.tins.2012.02.001 


\section{References}

Wagenaar, W. A. (2009). Expert witness in international war crimes tribunals. Psychology, Crime \& Law, 15, 583-596. doi:10.1080/10683160802438338

Wagenaar, W. A., \& van der Schrier, J. H. (1996). Face recognition as a function of distance and illumination: A practical tool for use in the courtroom. Psychology, Crime \& Law, 2, 321332. doi:10.1080/10683169608409787

Weber, N., \& Brewer, N. (2003). The effect of judgment type and confidence scale on confidenceaccuracy calibration in face recognition. Journal of Applied Psychology, 88, 490-499.

Weber, N., \& Brewer, N. (2004). Confidence-accuracy calibration in absolute and relative face recognition judgments. Journal of Experimental Psychology: Applied, 10, 156-172. doi:10.1037/1076-898x.10.3.156

Weber, N., \& Brewer, N. (2006). Positive versus negative face recognition decisions: Confidence, accuracy, and response latency. Applied Cognitive Psychology, 20, 17-31. doi:10.1002/acp.1166

Weber, N., Brewer, N., Wells, G. L., Semmler, C., \& Keast, A. (2004). Eyewitness identification accuracy and response latency: The unruly 10-12 second rule. Journal of Experimental Psychology: Applied, 10, 139-147. doi:10.1037/1076-898X.10.3.139

Wells, G. L. (1978). Applied eyewitness-testimony research: System variables and estimator variables. Journal of Personality and Social Psychology, 36, 1546-1557. doi:10.1037/00223514.36.12.1546

Wells, G. L., Kovera, M. B., Douglass, A. B., Brewer, N., Meissner, C. A., \& Wixted, J. T. (2020). Policy and procedure recommendations for the collection and preservation of eyewitness identification evidence. Law and Human Behavior, 44, 3-36. doi:10.1037/Ihb0000359

Wells, G. L., \& Penrod, S. D. (2011). Eyewitness identification research: Strengths and weaknesses of alternative methods. In B. Rosenfeld \& S. D. Penrod (Eds.), Research methods in forensic psychology (pp. 237-256). Hoboken, NJ, US: John Wiley \& Sons Inc.

Wells, G. L., Memon, A., \& Penrod, S. D. (2006). Eyewitness evidence: Improving its probative value. Psychological Science in the Public Interest, 7, 45-75. doi:10.1111/j.15291006.2006.00027.x

Wells, G., \& Olson, E. (2003). Eyewitness testimony. Annual Review of Psychology, 54, 277-295. doi:10.1146/annurev.psych.54.101601.145028

Wells, G.L. (2014). Eyewitness identification: Probative value, criterion Shifts, and policy regarding the sequential lineup. Current Directions in Psychological Science, 23, 11-16. doi:10.1177/0963721413504781

Wessling, K. S., Huber, J., \& Netzer, O. (2017). MTurk character misrepresentation: Assessment and solutions. Journal of Consumer Research, 44, 211-230. doi:10.1093/jcr/ucx053

Wilson J.P., Bernstein M.J., \& Hugenberg K. (2016) A synthetic perspective on the own-race bias in eyewitness identification. In Bornstein B., \& Miller M. (Eds), Advances in psychology and law, Vol. 2 (pp 241-270). doi:10.1007/978-3-319-43083-6_8 
Wixted, J., \& Mickes, L. (2014). A signal-detection-based diagnostic-feature-detection model of eyewitness identification. Psychological Review, 121, 262-276. doi:10.1037/a0035940

Wright, A. M., \& Holliday, R. E. (2007). Enhancing the recall of young, young-old and old-old adults with cognitive interviews. Applied Cognitive Psychology, 21, 19-43. doi:10.1002/acp.1260

Wright, K., Hull, J., Hughes, R., Ronda, J., \& Czeisler, C. (2006). Sleep and wakefulness out of phase with internal biological time impairs learning in humans. Journal of Cognitive Neuroscience, 18, 508521. doi:10.1162/jocn.2006.18.4.508

Wright, K., Lowry, C., \& LeBourgeois, M. (2012). Circadian and wakefulness-sleep modulation of cognition in humans. Frontiers in Molecular Neuroscience, 5, 1-12.

doi:10.3389/fnmol.2012.00050

Yang, L., Hasher, L., \& Wilson, D. (2007). Synchrony effects in automatic and controlled retrieval. Psychonomic Bulletin \& Review, 14, 51-56. doi:10.3758/bf03194027

Yonelinas, A. P. (2002). The nature of recollection and familiarity: A review of 30 years of research. Journal of Memory and Language, 46, 441-517. doi:10.1006/jmla.2002.2864

Yonelinas, A. P., \& Jacoby, L. L. (2012). The process-dissociation approach two decades later: Convergence, boundary conditions, and new directions. Memory \& Cognition, 40, 663680. doi:10.3758/s13421-012-0205-5

Yoon, C. (1997). Age differences in consumers' processing strategies: An investigation of moderating influences. Journal of Consumer Research, 24, 329-342. doi:10.1086/209514

Zeger, S., Liang, K., \& Albert, P. (1988). Models for longitudinal data: A Generalized Estimating Equation approach. Biometrics, 44, 1049-1060. 10.2307/2531734 


\section{Summary}

Chapter 1: Introduction. This chapter provides an introduction of the phenomenon of circadian variations in memory performance. I describe the mechanism of functioning of our circadian clock and review the literature on inter-individual differences in the timing of circadian phases. Importantly, the circadian clock determines the hours of the day when we reach or peak cognitive performance. Optimal performance can be achieved during periods of the day that are aligned with circadian phases of peak alertness. I review previous studies showing time-of-day variations in memory performance and describe potential relevance of these findings to different aspects of memory functioning in eyewitnesses.

Chapter 2: Circadian variations in eyewitness memory performance. This chapter presents an initial attempt to investigate time-of-day optimality effects across three domains relevant to eyewitness memory performance. For this purpose, I recruited one-hundred-and-three participants with morning and evening chronotype and tested each participant in the morning and in the evening. In each session, participants encoded a digitally recorded stimulus event that depicted a staged theft. At test, they provided free recall narratives about what they had seen, answered cued questions about the incident and the appearance of the people involved, and identified the individuals they saw in the stimulus event from target-present and target-absent lineups. I hypothesized that participants would provide more accurate free narratives and answers to cued questions when tested at their optimal compared to the non-optimal time of day. I also expected higher identification accuracy in the optimal compared to the non-optimal sessions. I hypothesized that non-optimal testing would weaken the postdictive value of confidence and decision times. Finally, I administered a visual version of the DRM paradigm, expecting that non-optimal testing would result in higher of false memory rates.

Accuracy in free narratives and answers to cued questions and false memory rates in the DRM task did not vary across the optimality conditions. Unexpectedly, participants showed higher identification accuracy in target-present but not target-absent lineups at non-optimal compared to the optimal time of day. Target selection rates were lower in optimal sessions, while foil selections did not vary as a function of testing optimality. The decision time-accuracy relationship commonly found in choosers was diminished at the non-optimal time of day. The confidence-accuracy relationship was not affected by testing optimality, with some evidence for superior calibration of confidence judgments at non-optimal compared to optimal sessions.

Chapter 3: Chronotype and time-of-day effects on eyewitness identification accuracy and its postdictors. Following our unexpected findings showing superior identification performance at nonoptimal compared to the optimal time of day, I conducted Experiment 2 to further investigate synchrony effects in lineup identification performance. I tested three-hundred-and-twenty-four participants with morning or evening time-of-day preference recruited with the help of the Amazon MTurk platform. Participants were tested either at their optimal or the non-optimal time of day. After encoding a staged stimulus event, participants identified people involved in the depicted incident from target-present and target-absent lineups. I was interested in testing whether our surprising findings from Experiment 1 
would replicate in a more diverse MTurk sample. Results showed no significant differences in identification accuracy between optimal and non-optimal sessions. Interestingly, evening-type participants were significantly more accurate in their identification decisions compared to morning types. Confidence was a stronger of accuracy in choosers at non-optimal compared to the optimal time of day. Decision times were not predictive of accuracy, likely due to the specifics of online testing environment.

Chapter 4: Circadian effects on face recognition and source memory performance. Long-term memory performance is generally better at the optimal compared to the non-optimal time of day. However, circadian fluctuations in face recognition performance received no attention in the literature to date. In Experiment 3, I used the face recognition paradigm to test possible synchrony effects in face recognition and source memory performance. Ninety-one morning- and evening-type participants were tested either in the morning or in the evening. Participants were presented with two sets of face stimuli in two contexts. One set of faces was embedded in a crime-related scenario, whereas the other set was presented in a neutral scenario. I hypothesised that general recognition performance would be better at the optimal compared to the non-optimal time of day. I also expected participants to be better at excluding familiar but irrelevant stimuli in the optimal compared to the non-optimal sessions. Results showed that overall recognition performance was unaffected by testing optimality. When asked to exclude familiar but irrelevant faces, participants showed only slight benefit from non-optimal testing that was not statistically significant. Although the current findings show no evidence for the effects of circadian arousal on face recognition and source memory for faces, future research is necessary to explore the underlying mechanisms of time-of-day effects in face recognition and source memory performance in vulnerable witnesses.

Chapter 5: General Discussion. In the final chapter, I attempt to synthesise the results obtained across all experiments within the research programme. The discrepancies between our findings and previous research are analysed from the viewpoint of divergence of methodological approaches to studying eyewitness memory performance from generic memory research methodology used in prior studies. An important line of discussion revolves around the problem of integrating findings obtained in generic laboratory experiments into applied contexts, such as eyewitness memory domain. The chapter outlines exciting directions for future research, such as the study of unorthodox synchrony effects in processing of faces and the effect of non-optimal testing on postdictors of eyewitness identification performance. I outline initial conclusions regarding the role circadian variations in arousal play in the eyewitness memory field, with an extra emphasis on the importance of replication of our findings due to the novelty of the research line. Finally, I discuss limitations of the current research programme and provide methodological suggestions for future study of the circadian effects in eyewitness memory performance. 


\section{Valorisation}

\section{Relevance}

It is difficult to find an area of our life that is not affected by our body clock. From oscillations on a molecular level to fluctuations in complex cognition, the circadian clock plays an important role in almost all areas of our functioning. Synchronising important activities with these cycles can result in improved productivity. Mismatch with our internal rhythms, on the contrary, can lead to undesirable effects, such as declines in performance. It is important to take into account these fluctuations when planning highly demanding activities. The eyewitness memory domain represents an area in which optimal performance is critical. It is important to identify factors that could potentially worsen the memory for the crime and the people involved. This information can be taken into account when assessing the probative value of evidence that witnesses provide. Time of day could be among such factors; therefore, it is important to know whether memory for events and people involved can be worse at hours of the day that are misaligned with our internal body rhythms. We also aim to create conditions for witnesses to provide as accurate and complete testimony as possible. From this point of view, it is important to know if the beneficial influence of peak performance hours can be used to provide additional retrieval support in witnesses. It is also critical to know whether there are hours of the day when obtaining information from witnesses would be least efficient and refrain from scheduling eyewitness interviews and identification procedures during these periods. Therefore, the current thesis is relevant as it extends the existing chronobiological research to the applied eyewitness memory field.

\section{Target Population and Products}

A wide array of factors can affect how well we remember events we witnessed. These factors should be taken into account when assessing the evidential value of information provided by eyewitnesses. Law enforcement agencies can also benefit from knowledge about these factors in improving the protocols for obtaining evidence from eyewitnesses. One of the aims of the eyewitness memory field, and, by extension, this thesis, is to help policy makers and legal practitioners navigate in the ocean of factors that can potentially affect memory functioning and identify those that are most relevant to real-life eyewitnesses. This thesis investigates of the role of time of day as one of the factors that can be relevant to the eyewitness memory domain. Daily fluctuations in memory performance are common: our memory is often best during hours of the day that coincide with circadian peaks in arousal. This thesis shows that young healthy witnesses are not subject to such classical 24-hour variations in memory performance. These findings can be informative to lawyers, police, judges and juries to determine whether time of day can affect the probative value of a piece of evidence obtained from an eyewitness. I also found no evidence suggesting the need in the change of policy in terms of the time of day when witness interviews are conducted or when lineup identifications are administered. This knowledge can guide policy makers to prioritize the necessary changes in protocols for obtaining evidence from witnesses. 


\section{Innovation}

The aim of this thesis was to investigate variations in eyewitness memory performance across the day caused by the circadian rhythm. To the best of my knowledge, this is the first published comprehensive attempt to study the effect of our body clock on the memory of witnesses. Specifically, the current thesis presents the very first published attempts to study circadian variations in identification performance using the so-called eyewitness identification paradigm. This set of methodologies is designed to reproduce the conditions real-life eyewitnesses encounter in psychological laboratory. The eyewitness memory paradigm greatly differs from methodologies of previous research into the circadian effects on memory performance in the stimuli type, encoding and retrieval instructions. I believe these factors account for the fact that non-optimal testing did not affect performance of our mock witnesses in the same way as in previously conducted experiments using the verbal learning paradigm, in which participants study words, sentences and short stories. Further, this thesis is the first to examine time-ofday effects on postdictors of eyewitness identification accuracy, such as confidence and decision times. The results in terms of confidence are especially interesting as they show that witnesses may take into account the fact that their memory performance can be lower at certain hours of the day. Importantly, the effect of circadian variations on face recognition performance in arousal has not been studied before. Processing of faces is a highly specialized cognitive function and differs in many aspects from processing of non-facial stimuli. It is important to understand whether time-of-day affects our memory for faces differently compared to other types of information.

\section{Implementation and Knowledge Dissemination}

The findings of this dissertation were made accessible through different channels. All of the experiments comprising this thesis have been presented at international conferences on psychology and law and applied cognition in Belgium, Finland and the USA. The studies in Chapters 2, 3 and 4 are under review to be published in academic journals. Publication of this research is important to communicate the findings to researchers who are seeking to understand how chronobiological phenomena translate into applied settings such as the eyewitness memory domain. The current findings have also been mentioned in lectures to psychology students and to fellow researchers via social media (e.g. Twitter). 


\section{Acknowledgments}

This thesis wouldn't have existed without amazing colleagues, friends and family who surrounded me with love and support throughout the process of obtaining my PhD. First and foremost, I couldn't have been luckier with the people who supervised me. Their academic brilliance, integrity, values, and extensive knowledge have set a high standard for my future academic endeavours. I am so grateful for their effort and patience in guiding me through a difficult process of becoming an independent researcher. Melanie, you treated me as a fellow academic from my very first steps in this PhD program and I had no other choice but to meet the standard. You softly helped me navigate my ideas while allowing plenty of room for creativity and individual expression. Lorraine, your passion for research is simply inspiring. You always had the right words of encouragement and it simply worked miracles at times. This made an immense difference.

I was lucky to be surrounded by many brilliant academics at two universities in which this research was conducted. Thank you to everyone at the Forensic Psychology section at Maastricht University and the Psychology department at University of Portsmouth for your feedback and advice, and for creating an inspiring and pleasant environment to grow professionally. I would especially like to thank my Maastricht University promotors, Henry and Harald, and my University of Portsmouth examiners Graham Pike, Paul Morris, Ryan Fitzgerald and Hartmut Blank for their valuable time and useful feedback. Maartje, thank you for sharing your expertise as a linguist, and for co-founding the writing club.

I was also extremely lucky with the companions for this exciting adventure. Kathy, Bri, and Ale, it means so much to me that we went through everything side-by-side. You always had my back when I needed it and it's difficult to imagine completing this PhD programme without you. I shared so many defining moments with each one of you. I feel like you are my academic family, and this is more important than any academic credentials I could have gained in the program. Nina, our inspiring discussions and your brilliant feedback helped me so many times. As a successful program graduate, you always found time and patience to share your experience with us. Your helped me in so many ways throughout this PhD. I am lucky to be your friend. Irena, I still recall our first encounter with a smile; you brought a smile to my face so many times afterwards. Thank you for your feedback, help and all the wise advice I've received from you. Renan, you were always willing to share your vast knowledge of our field; it was great becoming your officemate at UoP. Tameka, Aleks, Nicole, Carey, Nathalie, David, Shiri, Meghana, Minhwan, Bruna, Jenn, Nkansah, Enide, Joanne, it was my pleasure to be a part of this program with every one of you.

I was lucky to be surrounded by friends who supported me and believed in me. Markus, Umberto, Robin, thank you for long conversations and debates that often helped me formulate my abstract ideas. You could always find a new angle to any topic and it is always inspiring to speak to you. Olya, Mirella, Weronika, you are among the few people whose mere presence makes a huge difference. Thank you for your inner light and all the care and support I've received from you. I would like to thank 
my mentor Boris Khersonsky for his guidance and wisdom. Mom, dad, your love and support simply have no limits. Thank you for everything.

And last but not least, I would like to gratefully acknowledge the founders of the Erasmus Mundus Joint Doctorate The House of Legal Psychology and thank European Commission for providing funding for this outstanding PhD programme. I am grateful to everyone who contributed to designing this programme, helped organize and run it, and offered their time, resources and expertise to provide us with this incredible learning experience. 
Curriculum Vitae 


\section{Curriculum Vitae}

Sergii Yaremenko was born on 27 June, 1990, in Vinnytsia, Ukraine. He completed his primary and secondary education in his hometown in Ukraine. In 2012, he graduated with a master degree in Criminal Law from the National University 'Odessa Law Academy'. He continued his studies at the Odessa Mechnikov University to obtain his second Master's degree in Clinical Psychology in 2015. In 2016, he enrolled in the PhD programme with the Erasmus Mundus Joint Doctorate Program in Legal Psychology. His project on circadian variations in eyewitness memory performance was supervised by Dr. Melanie Sauerland, Prof. Lorraine Hope, Prof. Henry Otraar and Prof. Harald Merckelbach. During his PhD programme, he was primarily based at Maastricht University in the Netherlands. In 2017, he joined the Department of Psychology at the University of Portsmouth for 6 months to continue his research with the Hope Applied Cognition (HAC) Lab. 
Publications 


\section{Publications}

Yaremenko, S., Sauerland, M., Hope, L. (2020) Circadian variations in eyewitness identifications: Identification performance is not affected by time-of-day optimality. Manuscript submitted for publication, revision invited.

Yaremenko, S., Sauerland, M., Hope, L. (2020) Time-of-day effects on eyewitness reports in morning and evening types. Manuscript submitted for publication.

Yaremenko, S., Sauerland, M., Hope, L. (2020) Circadian rhythm and memory performance: no time-ofday effect on face recognition. Manuscript submitted for publication, revisions invited.

\section{Conference Presentations}

Yaremenko, S., Sauerland, M., Hope, L. (June 2019) Chronotype and time-of-day effects on eyewitness identification performance. Poster presentation at the thirteenth biennial conference of the Society for Applied Research in Memory and Cognition (SARMAC) in Cape Cod, USA.

Yaremenko, S., Sauerland, M., Hope, L., Merckelbach, H. (June 2018) Chronotype and time-of day effects on eyewitness memory. Presentation at the annual meeting for the European Association for Psychology and Law (EAPL), Turku, Finland.

Yaremenko, S., Sauerland, M., Hope, L., Merckelbach, H. (May 2017) Chronotype and time-of day effects on eyewitness memory. Presentation at the annual meeting for the European Association for Psychology and Law (EAPL), Mechelen, Belgium.

\section{Grants, Honors, and Scholarships}

Awarded APA Div. 41 AP-LS Grants-in-Aid, 2018

Best student presentation award, European Association of Psychology and Law Conference, 2018 
Appendices 


\section{Appendices}


Appendices 


\section{Appendix A: Cued questions about the event and people involved (Chapter 2; Experiment 1)}

\section{Cued questions about the event}

1. Describe any interactions the thief/thieves had with the other people in the film.

2. How did the thief/thieves get the opportunity to steal the wallet?

3. What was the victim doing when the thief/thieves stole the wallet?

4. How long did the theft last?

5. Were there any accomplices?

6. What did the thief/thieves do with the stolen wallet?

7. What did the victim do when she/he realized the wallet was stolen?

8. How many people did you see in the film?

9. Is there any other information you would like to share with us about the event that we have not asked you about?

\section{Cued questions about the thief}

1. How old was the thief? (Enter one number, not a range)

2. How tall was the thief in $\mathrm{cm}$ ? (Enter one number, not a range)

3. Describe the thief's build.

4. Describe the thief's clothing.

5. Describe the thief's hair color.

6. Describe the thief's hairstyle.

7. Describe the thief's face shape.

8. Did you notice any special features in the appearance of the thief?

9. Did the thief wear something on his/her head? If yes, what?

10. Did the thief wear glasses? If yes, what did they look like? 


\section{Appendix B: Counterbalancing plan for face stimuli in Chapter 2; Experiment 3)}

Supplementary Table 1

Counterbalancing plan for face stimuli presented during encoding, face recognition and source memory task.

Optimal time of day

Non-optimal time of day

\section{Encoding}

\begin{tabular}{|c|c|c|c|}
\hline Crime-related scenario - Set 1 & Crime-related scenario - Set 2 & Crime-related scenario - Set 1 & Crime-related scenario - Set 2 \\
\hline Supermarket scenario - Set 2 & Supermarket scenario - Set 1 & Supermarket scenario - Set 2 & Supermarket scenario - Set 1 \\
\hline
\end{tabular}

Face recognition task

\begin{tabular}{|c|c|c|c|c|c|c|c|c|c|c|c|c|c|c|c|}
\hline Set $1 A$ & Set $1 B$ & Set $1 \mathrm{~A}$ & Set $1 B$ & Set $2 \mathrm{~A}$ & Set 2B & Set $2 \mathrm{~A}$ & Set $2 B$ & Set $1 \mathrm{~A}$ & Set $1 B$ & Set $1 \mathrm{~A}$ & Set $1 B$ & Set $2 \mathrm{~A}$ & Set $2 B$ & Set $2 \mathrm{~A}$ & Set $2 B$ \\
\hline Set $2 A$ & Set $2 B$ & Set $2 B$ & Set $2 A$ & Set $1 \mathrm{~A}$ & Set $1 B$ & Set $1 B$ & Set $1 \mathrm{~A}$ & Set $2 A$ & Set $2 B$ & Set $2 B$ & Set $2 A$ & Set $1 \mathrm{~A}$ & Set $1 B$ & Set $1 B$ & Set $1 \mathrm{~A}$ \\
\hline
\end{tabular}

Distractor Distractor Distractor Distractor Distractor Distractor Distractor Distractor Distractor Distractor Distractor Distractor Distractor Distractor Distractor Distractor

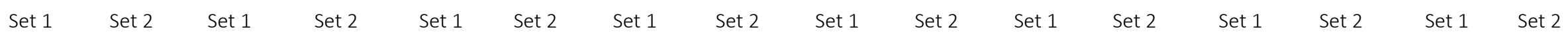

Source memory task

\begin{tabular}{|c|c|c|c|c|c|c|c|c|c|c|c|c|c|c|c|}
\hline Set 1B & Set $1 \mathrm{~A}$ & Set $1 B$ & Set $1 \mathrm{~A}$ & Set 2B & Set $2 \mathrm{~A}$ & Set 2B & Set $2 \mathrm{~A}$ & Set 1B & Set $1 \mathrm{~A}$ & Set 1B & Set $1 \mathrm{~A}$ & Set $2 B$ & Set $2 \mathrm{~A}$ & Set $2 B$ & Set $2 A$ \\
\hline Set $2 B$ & Set $2 \mathrm{~A}$ & Set $2 \mathrm{~A}$ & Set $2 B$ & Set $1 B$ & Set $1 A$ & Set $1 \mathrm{~A}$ & Set $1 B$ & Set $2 B$ & Set $2 \mathrm{~A}$ & Set $2 A$ & Set $2 B$ & Set $1 B$ & Set $1 A$ & Set $1 \mathrm{~A}$ & Set $1 B$ \\
\hline
\end{tabular}

Distractor Distractor Distractor Distractor Distractor Distractor Distractor Distractor Distractor Distractor Distractor Distractor Distractor Distractor Distractor Distractor

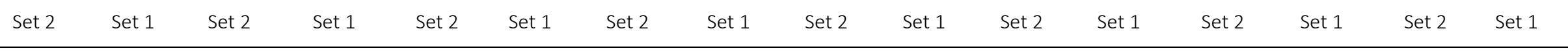

Note. Sets 1A, 1B, 2A, 2B contained 8 faces each. Each distractor set contained 16 faces. 


\title{
Appendix C - Ethical Approval
}

The protocol of experiments presented in this thesis was examined and approved by the Ethical Review Committee Psychology and Neuroscience (ERCPN) of Maastricht University. Below is the official letter of approval of the research line. Each individual study within the research line received individual approval from Maastricht University ECP.

\section{Maastricht University in Letrining!}

\author{
Board of FPN \\ Universiteit Maastricht \\ Postbus 616 \\ 6200 MD Maastricht
}

\begin{tabular}{|c|c|c|}
\hline & & $\begin{array}{l}\text { Committee } \\
\text { Neuroscience }\end{array}$ \\
\hline Dur reference & $\begin{array}{l}\text { direct dial } \\
0031.43 .388 .4008\end{array}$ & Maastricht \\
\hline
\end{tabular}

Dear Board,

After examination of the research protocol entitled "Chronotype in eyewitness performance research line", submitted by Sergii Yaremenko, the Ethical Review Committee Psychology and Neuroscience (ERCPN) came to the conclusion that there are no objections to the execution of the research project as described in the said protocol with regard to the review framework used. The applicant has been informed that:

1. Approval has been granted for a period of five years, with the possibility to prolong.

2. If the approval has been granted for a research line, each individual study within this line must be notified to the ERCPN using the form provided on the website. This does not include studies which are reviewed by a proposal committee (i.a. fMRI, EEG and TMS).

3. Changes to the approved research protocol must be submitted by the ERCPN.

4. The reference number should be mentioned in all correspondence with the ERCPN.

5. The reference number must be indicated on all advertising communications to recruit participants.

Yours sincerely,

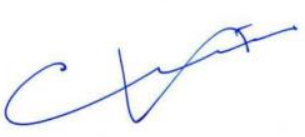

Prof. Dr. G. Kok Chair ERCPN

Cc. Sergii Yaremenko

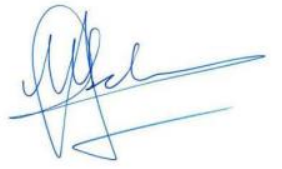

Mr. M. Schrijnemaekers Secretary ERCPN

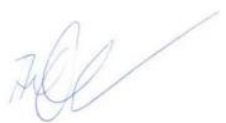

Prof. Dr. A.T. Sack Board of FPN

Bezoekadres Universiteitssingel 40
6229 ER Maastricht

Email adres
ercpn-fpn@maastrichtuniversity.nt
Voorzitter: G. Kok

Ambtelijk secretaris:
M. Schrijnemaekers 

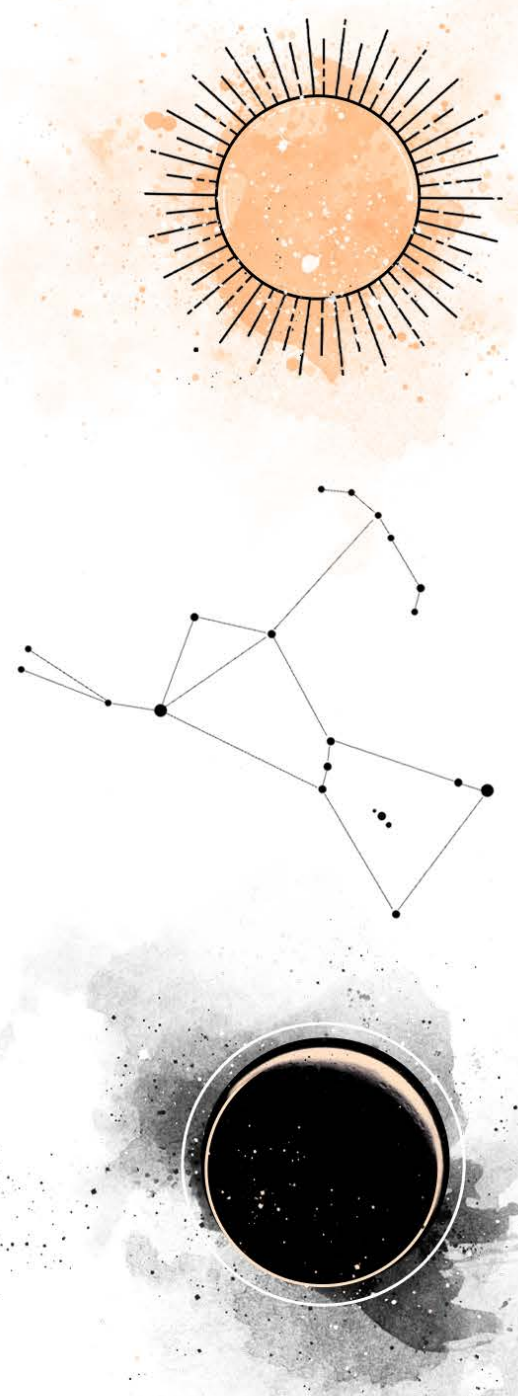

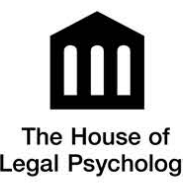

